DUST FORMATION AND EVOLUTION AROUND CARBON STARS

\author{
A Dissertation
}

presented to

the Faculty of the Graduate School

at the University of Missouri-Columbia

In Partial Fulfillment

of the Requirements for the Degree

Doctor of Philosophy

by

HANNAH GROOM

Dr. Angela Speck, Dissertation Supervisor

MAY 2018 
The undersigned, appointed by the dean of the Graduate School, have examined the dissertation entitled

\section{DUST FORMATION AND EVOLUTION AROUND CARBON STARS}

presented by Hannah Groom, a candidate for the degree of doctor of philosophy, and hereby certify that, in their opinion, it is worthy of acceptance.

\begin{tabular}{c}
\hline Professor Angela Speck \\
\hline Professor Linda Godwin \\
\hline Professor Sergei Kopeikin \\
\hline Professor Alina Zare
\end{tabular}


This book is for: my Grandpa, Jack, who showed what type of light I want to be in this world; my parents who always wished, 'when you get the choice to sit it out or dance, I hope you dance.' Lastly, to my haters, you're my biggest motivation and fans. 


\section{ACKNOWLEDGMENTS}

I would like to thank the three women and one activity ${ }^{1}$ in which helped me climb my personal "Mt. Everest". First, to my Sherpa, Dr. Angela Speck. Without her, I would have never known how to climb the mountain of a dissertation, or at least would have gotten lost along the way. When I wanted to quit and did not think it was worth it, she was there to help pick me up. We were a team and we conquered the mountain together. Second, Dr. Margaret Hill, she was the woman who showed me what physics was. Yes, it might have been finding the frictional force of a seal sliding down a ramp at 35 degrees. Without her setting the spark, I would have never dreamt to begin this journey. Also, just like all good road trips need an epic sound track, so do life journeys. So this is a shout out to Taylor Swift. Lastly, rugby became a passion in which I would have never found without starting this trek. I have gained black eyes, bruises and also the best family ever. It is an emotional outlet and also an eye opener to applied physics in real life applications.

\footnotetext{
${ }^{1}$ Maybe also George W. Bush, the $43^{r d}$ Commander-in-Chief, whose impressive career advancement despite remedial language skills inspired me to believe I could finish my dissertation.
} 


\section{Table of Contents}

Acknowledgements $\quad$ ii

List of Tables

List of Figures viii

$\begin{array}{ll}\text { Abstract } & \text { xvi }\end{array}$

1 Introduction 1

1.1 Why Do We Care About Dust? . . . . . . . . . . . . . . . . 1

2 Stellar Evolution and AGB Stars 5

2.1 Introduction . . . . . . . . . . . . . . . . 5

2.1.1 Surface Temperature and Size ............ 6

2.1.2 Distance ........................ 7

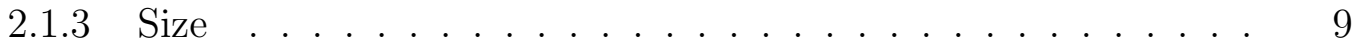

2.1.4 Hertzsprung-Russel Diagram . . . . . . . . . . . . . . . . 9

2.2 Stellar Evolution . . . . . . . . . . . . . . . . 10

2.2.1 Birth of Stars . . . . . . . . . . . . . . . . . 11

2.2 .2 Main Sequence . . . . . . . . . . . . . . . 18 
2.2 .3 Red Giants . . . . . . . . . . . . . . . . . . . . 21

2.2 .4 Horizontal Branch . . . . . . . . . . . . . . . . . 28

$2.2 .5 \quad$ Asymptotic Giant Branch _. . . . . . . . . . . . . . . . 29

2.3 Death of a Star . . . . . . . . . . . . . . . . . . . . . 39

2.3 .1 Planetary Nebulae . . . . . . . . . . . . . . . . . . . . 40

2.3.2 White dwarf . . . . . . . . . . . . . . . . . 41

2.3.3 Conclusion of Stellar Evolution _. . . . . . . . . . . . 41

3 Optical Properties $\quad 43$

3.1 Introduction $\ldots \ldots \ldots \ldots \ldots \ldots \ldots \ldots \ldots \ldots$

3.2 Classical dispersion analysis $\ldots \ldots \ldots \ldots \ldots$

3.2 .1 Lorentz Model . . . . . . . . . . . . . . . . . . . . . . . . . 45

3.3 Light Propagation Through Dust . . . . . . . . . . . . . 50

3.3 .1 Extinction . . . . . . . . . . . . . . . . . . 51

$3.3 .2 \quad$ Spectral Analysis . . . . . . . . . . . . . . . . . 56

4 Observations of Carbon Stars and the Dust Condensation Sequence 58

4.1 Introduction $\ldots \ldots \ldots \ldots \ldots \ldots \ldots \ldots \ldots$

$4.1 .1 \sim 11 \mu \mathrm{m}$ Feature . . . . . . . . . . . . . . . . . 59

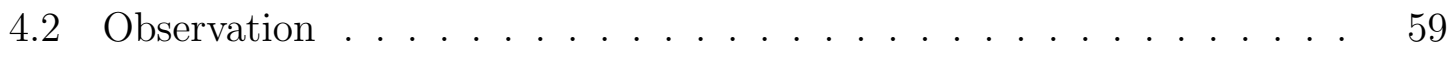

4.3 Radiative Transfer Modeling . . . . . . . . . . . . . . . . . 60

4.3.1 Radial Dust Density Distribution . . . . . . . . . . . . 61

4.3.2 Modeling Grain-Size Distributions . . . . . . . . . . . . . 61

4.3 .3 Parameter Space Investigated $\ldots \ldots \ldots 2$ 
4.4 Determining the Dust Condensation Temperature . . . . . . . . . 65

4.4.1 Theoretical Dust Condensation Models . . . . . . . . . . . 65

4.4.2 Constraining Dust Composition . . . . . . . . . . . . . 70

5 Transfer Modeling Selection and Results $\quad 73$

5.1 Target Selection and Observations . . . . . . . . . . . . 73

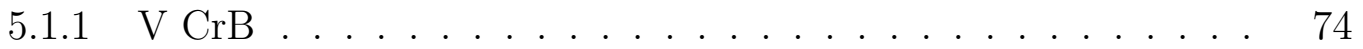

5.2 Modeling Results . . . . . . . . . . . . . . . . 76

5.2.1 Correlations Between Collated and Model Parameters . . . . . 93

5.2.2 Correlations Between Model Parameters . . . . . . . . . . 93

5.2 .3 Discussion ........................ 135

6 Summary and Future Work 137

6.1 Conclusion . . . . . . . . . . . . . . . . . . . . 137

6.1.1 Future Work . . . . . . . . . . . . . . . . . . . 138

6.1 .2 Future Work . . . . . . . . . . . . . . . . 138

6.1.3 Future Work . . . . . . . . . . . . . . . . . . 139

$\begin{array}{lr}\text { Reference } & 140\end{array}$

Vita 


\section{List of Tables}

4.1 Target Sources . . . . . . . . . . . . . . . . . . . . . 60

5.1 Sample of optically thin carbon stars $\ldots \ldots \ldots \ldots$

5.2 Collected data for our carbon star sample $\ldots \ldots \ldots \ldots \ldots$

5.3 Collected data for our carbon star sample $\ldots \ldots \ldots \ldots$

5.4 Parameters for graphite single shell modeling. . . . . . . . . . 86

5.5 Parameters for AmC single shell modeling. . . . . . . . . . . . 87

5.6 Parameters for iron single shell modeling. . . . . . . . . . . . . 88

5.7 Parameters for 2-shell Graphite modeling. . . . . . . . . . . . 89

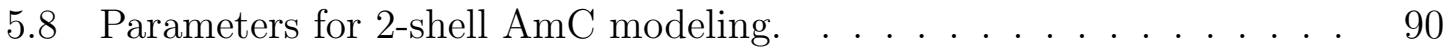

5.9 Parameters for graphite/Fe single shell modeling. . . . . . . . . . 91

5.10 Parameters for AmC/Fe single shell modeling. . . . . . . . . . . . 92

5.11 Correlations amongst collated parameters . . . . . . . . . . . 95

5.11 Continued. Correlations amongst collated parameters . . . . . . . 96

5.11 Continued. Correlations amongst collated parameters . . . . . . . 97

5.11 Contindued. Correlations amongst collated parameters _. . . . . . 98

5.12 Correlations between modeled and collated parameters for single shell

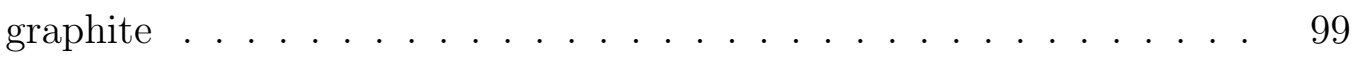


5.12 Continued. Correlations between modeled and collated parameters for single shell graphite . . . . . . . . . . . . . . . . . . . . 100

5.13 Correlations between modeled and collated parameters for single shell graphite . . . . . . . . . . . . . . . . 101

5.13 Continued. Correlations between modeled and collated parameters for single shell AmC . . . . . . . . . . . . . . . . . . . . . . . 102

5.14 Correlations between modeled and collated parameters for single shell iron

5.14 Continued. Correlations between modeled and collated parameters for single shell iron . . . . . . . . . . . . . . . . .

5.15 Correlations between modeled and collated parameters for single shell graphite $/ \mathrm{Fe} \ldots \ldots \ldots \ldots \ldots \ldots$

5.15 Continued. Correlations between modeled and collated parameters for single shell graphite/Fe . . . . . . . . . . . . . . . . . . . . 106

5.16 Correlations between modeled and collated parameters for single shell $\mathrm{AmC} / \mathrm{Fe} \ldots \ldots \ldots \ldots \ldots 7 . \ldots \ldots \ldots$

5.16 Continued. Correlations between modeled and collated parameters for single shell AmC/Fe . . . . . . . . . . . . . . . . . . . . 108

5.17 Correlations Amongst Collated 2-Shell Parameters . . . . . . . . . . . 109

5.17 Continued. Correlations Amongst Collated 2-Shell Parameters . . . . 113

5.18 Correlations between modeled and collated parameters for 2 shell graphite120

5.18 Continued. Correlations between modeled and collated parameters for 2 shell graphite . . . . . . . . . . . . . . . . . . 121 
5.19 Correlations between modeled and collated parameters for 2 shell AmC 122

5.19 Conintued. Correlations between modeled and collated parameters for 2 shell $\mathrm{AmC}$. . . . . . . . . . . . . . . . . . . . 123

5.20 Correlations betweens the $\mathrm{SiC}$ Abundances for models with different modeled continuum dust species . . . . . . . . . . . . . . . . . . . . . 124

5.20 Continued. Correlations between the SiC Abundances for models with different modeled continuum dust species . . . . . . . . . . . . . . . . 125

5.20 Continued. Correlations betweens the SiC Abundances for models with different modeled continuum dust species . . . . . . . . . . . . . . 126

\section{List of Figures}

2.1 Stellar Parallax. The dark sphere represents the sun, the small light sphere is the Earth, and the dashed circle represents the Earth's orbit; $d$ is the average distance between Earth and the star being observed, and $\theta$ is the parallax angle. . . . . . . . . . . . . . . 7

2.2 Hertzsprung-Russell Diagram showing luminosity vs temperature. . . 10

2.3 Diagram of a main sequence star. The tiny core of the star is fusing hydorgen into helium, and is surrounded by a larger atmosphere that does not participate in the fusion process. This is not to scale. . . 
2.4 Diagram of a red gaint. The inert core of helium is surrounded by a shell where hydorgen is being fused into helium, which is surrounded by a much larger inert envelope. This is not to scale. . . . . . . . . 26

2.5 Diagram of an AGB star. The inert core of carbon is surrounded by a shell where helium is being fused into carbon, which is surrouneded by a shell where hydrogen is being fused into helium. All of this is surrounded by a much larger inert envelope. . . . . . . . . . . .

3.1 Light propagating through a slab of dust. Reference discussion for an

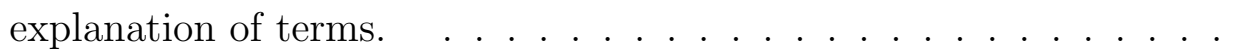

3.2 Diagram of absorption, refection and refraction of light . . . . . . . 55

4.1 Pressure-temperature space for the dust condensation region around carbon stars. The gray lines indicate the condensation temperature for a given pressure as calculated by Lodders \& Fegley (1995). The black lines indicate the P-T paths for the out-flowing gas from our sample stars. . . . . . . . . . . . . . . . .

5.1 The photometrics bands, phtoemertics measurements and the ISO SWS spectra of our target sample. The BV and JHK photometric bands (displayed as squares), the IRAS 12, 25, 60 and $100 \mu \mathrm{m}$ photometric measurements (displayed as circles) and the ISO SWS spectra (displayed as a dark dotted line). . . . . . . . . . . . . . . 
5.2 The photometrics bands, phtoemertics measurements and the ISO SWS spectra of our target sample. The BV and JHK photometric bands (displayed as squares), the IRAS 12, 25, 60 and $100 \mu \mathrm{m}$ photometric measurements (displayed as circles), the ISO SWS spectra (displayed as a dark dotted line), and and the modeled continuum (thick grey

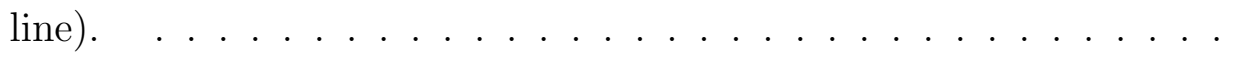

5.3 The photometrics bands, phtoemertics measurements and the ISO SWS spectra of our target sample. The BV and JHK photometric bands (displayed as squares), the IRAS 12, 25, 60 and $100 \mu \mathrm{m}$ photometric measurements (displayed as circles), the ISO SWS spectra (displayed as a dark dotted line), and and the modeled continuum (thick grey

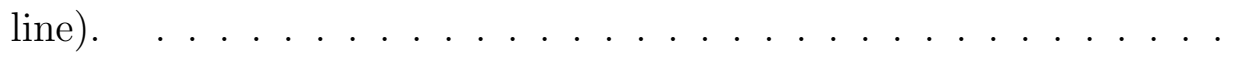

5.4 The photometrics bands, phtoemertics measurements and the ISO SWS spectra of our target sample. The BV and JHK photometric bands (displayed as squares), the IRAS 12, 25, 60 and $100 \mu \mathrm{m}$ photometric measurements (displayed as circles), the ISO SWS spectra (displayed as a dark dotted line), and and the modeled continuum (thick grey

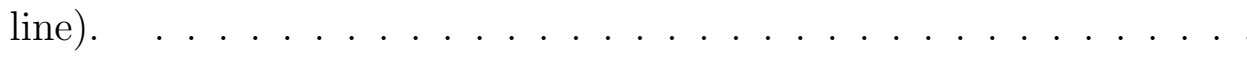


5.5 The photometrics bands, phtoemertics measurements and the ISO SWS spectra of our target sample. The BV and JHK photometric bands (displayed as squares), the IRAS 12, 25, 60 and $100 \mu \mathrm{m}$ photometric measurements (displayed as circles), the ISO SWS spectra (displayed as a dark dotted line), and and the modeled continuum (thick grey

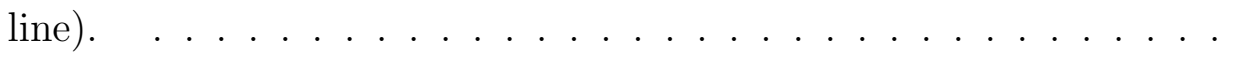

5.6 The photometrics bands, phtoemertics measurements and the ISO SWS spectra of our target sample. The BV and JHK photometric bands (displayed as squares), the IRAS 12, 25, 60 and $100 \mu \mathrm{m}$ photometric measurements (displayed as circles), the ISO SWS spectra (displayed as a dark dotted line), and and the modeled continuum (thick grey

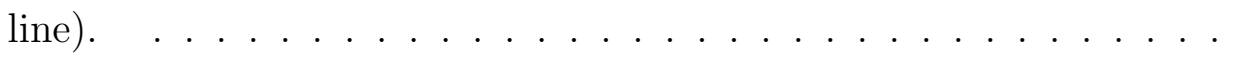

5.7 The photometrics bands, phtoemertics measurements and the ISO SWS spectra of our target sample. The BV and JHK photometric bands (displayed as squares), the IRAS 12, 25, 60 and $100 \mu \mathrm{m}$ photometric measurements (displayed as circles), the ISO SWS spectra (displayed as a dark dotted line), and and the modeled continuum (thick grey

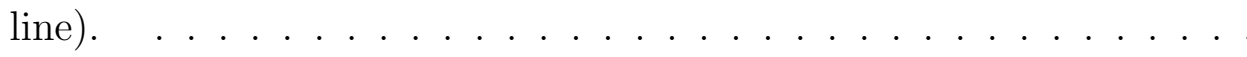


5.8 The photometrics bands, phtoemertics measurements and the ISO SWS spectra of our target sample. The BV and JHK photometric bands (displayed as squares), the IRAS 12, 25, 60 and $100 \mu \mathrm{m}$ photometric measurements (displayed as circles), the ISO SWS spectra (displayed as a dark dotted line), and and the modeled continuum (thick grey

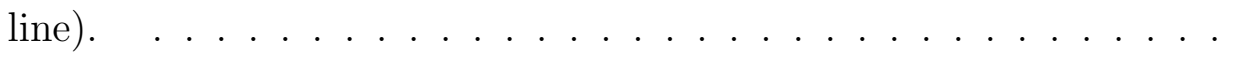

5.9 The correlations between the inner dust temperature and the change of temperature between the formation of graphite to $\mathrm{SiC}$ grains and the $\mathrm{C} / \mathrm{O}$ ratio and change of temperature between the formation of graphite to $\mathrm{SiC}$ grains for the collated parameters. . . . . . . . .

5.10 The correlations between the optical depth and the expasion velocity for AmC single shell models. . . . . . . . . . . . . . . . . . . . 109

5.11 The correlations between the amount of graphite grains and change of temperature between the formation of graphite to $\mathrm{SiC}$ grains and the amount of iron grains and change of temperature between the formation of graphite to $\mathrm{SiC}$ grains for graphite/Fe single shell models. . . 110

5.12 The correlations between the amount of graphite grains and $\mathrm{Fe} / \mathrm{H}$ ratio and the amount of iron grains and $\mathrm{Fe} / \mathrm{H}$ for graphite/Fe single shell models. . . . . . . . . . . . . . . . .

5.13 The correlations between the amount of graphite grains and ${ }^{12} \mathrm{C} /{ }^{13} \mathrm{C}$ ratio and the amount of iron grains and ${ }^{12} \mathrm{C} /{ }^{13} \mathrm{C}$ ratio for graphite $/ \mathrm{Fe}$ single shell models. . . . . . . . . . . . . . . . . . . . . . 112 
5.14 The correlations between the optical depth and gas-to-dust ratio for graphite/Fe single shell models. . . . . . . . . . . . . . . . . . . . . 113

5.15 The correlations between the amount of graphite grains and temperture of the inner dust shell and the amount of iron grains and temparture of the inner dust shell for graphite/Fe single shell models. . . . . . . 114

5.16 The correlations between the amount of iron grains and graphite grains and the $\mathrm{C} / \mathrm{O}$ ratio and the amount of graphite grains for graphite/Fe single shell models.

5.17 The correlations between the amount of iron grains and $\mathrm{AmC}$ grains and the $\mathrm{Fe} / \mathrm{H}$ ratio and the amount of $\mathrm{AmC}$ grains for $\mathrm{AmC} / \mathrm{Fe}$ single shell models. . . . . . . . . . . . . . . . . . . . . . . .

5.18 The correlations between the amount of $\mathrm{AmC}$ grains and change of temperature between the formation of graphite to $\mathrm{SiC}$ grains and the amount of iron grains and change of temperature between the formation of graphite to $\mathrm{SiC}$ grains for $\mathrm{AmC} / \mathrm{Fe}$ single shell models. . . . . 117

5.19 The correlations between the amount of $\mathrm{AmC}$ grains and the temperature of the inner dust shell and the amount of iron grains and the temperature of the inner dust shell for $\mathrm{AmC} / \mathrm{Fe}$ single shell models.

5.20 The correlations between the $\mathrm{Fe} / \mathrm{H}$ ratio and the amount of iron in AmC/Fe single shell models. . . . . . . . . . . . . . . . . . . . . . . 119

5.21 The correlations between the optical depth and gas-to-dust ratio for AmC/Fe single shell models. . . . . . . . . . . . . . . . . . . . 119 
5.22 The correlations between the abundances $\mathrm{SiC}$ grains and $\mathrm{C} / \mathrm{O}$ ratio in AmC/Fe single shell models. . . . . . . . . . . . . . . .

5.23 The correlations between the amount of graphite grains and the temperature of the inner dust shell and the amount of $\mathrm{SiC}$ grains and the temperature of the inner dust shell for graphite 2-shells models. . . . 128

5.24 The correlations between the amount of $\mathrm{AmC}$ grains and the temperature of the inner dust shell and the amount of $\mathrm{SiC}$ grains and the temperature of the inner dust shell for AmC 2-shells models.

5.25 The correlations between the amount of $\mathrm{AmC}$ grains and the $\mathrm{C} / \mathrm{O}$ ratio and the amount of $\mathrm{SiC}$ grains and the $\mathrm{C} / \mathrm{O}$ ratio shell for $\mathrm{AmC}$ 2-shells models.

5.26 The correlations between the abundances $\mathrm{SiC}$ grains in $\mathrm{AmC}$ and graphite single shell models and abundances $\mathrm{SiC}$ grains in iron and graphite single shell models.as . . . . . . . . . . . . . . .

5.27 The correlations between the abundances $\mathrm{SiC}$ grains in graphite/Fe and graphite single shell models and abundances $\mathrm{SiC}$ grains in $\mathrm{AmC} / \mathrm{Fe}$ and graphite single shell models. . . . . . . . . . . . . . . . . . . 132

5.28 The correlations between the abundances $\mathrm{SiC}$ grains in $\mathrm{Fe}, \mathrm{AmC}$ single shell models and abundances $\mathrm{SiC}$ grains in graphite/Fe and $\mathrm{AmC}$ and abundances $\mathrm{SiC}$ grains in $\mathrm{AmC} / \mathrm{Fe}$ and $\mathrm{AmC}$ single shell models. . . 133

5.29 The correlations between the abundances $\mathrm{SiC}$ grains in graphite/Fe and iron single shell models and abundances $\mathrm{SiC}$ grains in $\mathrm{AmC} / \mathrm{Fe}$ and iron single shell models. 
5.30 The correlations between the abundances $\mathrm{SiC}$ grains in $\mathrm{AmC} / \mathrm{Fe}$ and graphite/Fe single shell models for single shell models. . . . . . . . 135 


\section{Abstract}

Evolved intermediate-mass stars with carbon-to-oxygen ratios $(\mathrm{C} / \mathrm{O})$ above unity are known as carbon stars. Carbon stars are surrounded by dust shells dominated by carbon $(\mathrm{C})$ and silicon carbide $(\mathrm{SiC})$ grains. These $\mathrm{SiC}$ grains have a diagnostic spectral feature at $\sim 11 \mu \mathrm{m}$. We have selected a sample of 9 carbon stars with low mass-loss rates such that their dust shells are sufficiently optically thin to allow abundance analysis of the stars' photospheres. This allows the study of how atomic abundances affect dust formation around carbons stars. We present the result of radiative transfer modeling for these stars, and compare the resulting dust shell parameters to published abundance measurements. To constrain model parameters, we use published mass-loss rates, expansion velocities, and theoretical dust condensation models to estimate the dust condensation temperature, and spectral types to constrain stellar effective temperatures. We found significant correlations for the single-shell modeling with graphite/iron grains and amorphous carbon (AmC)/iron grains: (1) $\tau_{0.55 \mu m}$ and gas-to-dust ratio, (2) iron grains and graphite or AmC grains, (3) graphite or AmC grains and $\mathrm{Fe} / \mathrm{H}$, and (4) iron grains and $\mathrm{Fe} / \mathrm{H}$. For the collated data the significant correlations we found were: (1) for dust formation temperature and the change of temperature from the formation of graphite grains to the formation of $\mathrm{SiC}$ grains (2) $\mathrm{C} / \mathrm{O}$ and the change of temperature from the formation of graphite grains to the formation of $\mathrm{SiC}$ grains. Lastly, between the abundance of $\mathrm{SiC}$ grains when compared to the abundances of $\mathrm{SiC}$ grains in graphite, AmC, graphite and iron and $\mathrm{AmC}$ and iron grains models. This shows that there is no sensitivity in the 
continuum when choosing the type of carbon to model with. 


\section{Chapter 1}

\section{Introduction}

\subsection{Why Do We Care About Dust?}

The nitrogen in our DNA, the calcium in our teeth, the iron in our blood, the carbon in our apple pies were made in the interiors of collapsing stars. We are made of starstuff. - Carl Sagan

$$
\text { (Cosmos) }
$$

Hydrogen, helium and a tiny amount of lithium were created during the Big Bang; all other elements in the Universe come from nucleosynthesis inside of stars or collisions between stars. At the end of a stars life these elements are ejected, either slowly and gently in such as the case of low to intermediate mass stars, or violently in the case of high mass stars. Elements from both types of stars become part of the interstellar medium in the form of dust and gas and eventually could form new stars and planets.

With the emergence of infrared (IR) astronomy in late 1960s, the importance of 
dust particles in the Universe began to be discovered. Dust is an important component in many astrophysical environments, since it plays an essential role in star formation processes and contributes to different aspects of interstellar processes. Some processes are, such as gas heating and the formation of molecules. Since mass loss from evolved stars is driven by radiation pressure on dust grains, this is linked to the precise nature of the circumstellar dust. Dust needs to be understood, if we are to understand how it contributes to different aspects of astrophysics.

Factors in determining the mineralogy (chemical compositions and crystal structure), size and shape of dust grains are temperature, chemistry and density of the gas from which it forms. The chemistry determines the type of atoms available to form the dust particles. The density determines how likely these atoms are to collide with each other and create dust particles. The temperature determines which solid-state species are stable enough to form. The chemistry, density and temperature of a dust forming circumstellar shell are determined by the nature of the central star, including its metallicity (i.e. the fraction of mass in a star that is not hydrogen or helium) and the stars initial mass. An evolution in the nature of the dust produced may influence the stellar evolution, indicating a relationship between the changes in the star and dust formation in its circumstellar envelope. We cannot fully understand these stars until we determine the precise nature of their circumstellar dust.

The mineralogy of dust grains in space is typically studied by means of IR spectroscopy. The IR wavelength regime is well suited to determine mineral compositions since the dust in a circumstellar envelope absorbs the mostly optical light from the central star and re-radiates it at IR wavelengths. Dust particles of a given size, 
shape, temperature, structure, and composition, all have their own characteristic IR spectrum. We can then use the IR spectra of candidate dust species studied in the laboratory to identify IR spectral features observed in astronomical environments.

With the launch of ISO (Infrared Space Observatory), astromineralogy had a boom due to the superior spectral resolution available. Most ensuing studies have concentrated on oxygen rich stars, in part because of new features being discovered. However, this means that carbon rich stars have been neglected. In addition to spectroscopy, there are other constraints or lines of evidence that can be used to help our studies, which include spatial distributions of materials, theoretical models for dust formations and evidence from meteoritic studies of presolar grains.

Here, the main focus is with low to intermediate mass stars (LIMS), which have a mass between $0.8-8 \mathrm{M}_{\odot}$. About $95 \%$ of all stars are LIMS (Kwok, 2004). In particularly we will study LIMS late in their lives, when they are losing large amounts of mass and are forming circumstellar shells of gas and dust. This is called the Asymptotic Giant Branch (AGB) phase of the stars evolution.

When the star is in the AGB phase, it evolves chemically which has a strong influence on the type of dust produced. The most important parameter that determines the dust composition is the carbon-to-oxygen ratio $(\mathrm{C} / \mathrm{O})$ in the stars atmosphere. LIMS start out with oxygen-rich-atmospheres, but the star produces carbon deep inside, which can be dredged to the surface by powerful convection currents. For stars with masses between about $2-4 \mathrm{M}_{\odot}$, enough carbon can be mixed into the stars atmosphere by this process to make the $\mathrm{C} / \mathrm{O}$ greater than unity, we refer to these stars as carbon stars. Carbon rich stars make up about a third of all AGB stars in 
our own galaxy, though the exact proportion depends on the metallicity (Matsuura et al., 2005).

The $\mathrm{C} / \mathrm{O}$ ratio strongly affects the chemistry in the dust shell. This is because the stable carbon monoxide $(\mathrm{CO})$ molecules are one of the first species that forms out of the outflowing gas. The formation of $\mathrm{CO}$ will consume most of the carbon and oxygen in the gas, and the less abundant element will be entirely bound up by this process, making it unavailable for use in later chemistry.

For carbon-rich stars, the primary solid-state constituent of the dust shell is carbon, though the exact crystal structure of the carbon is uncertain. The second most commonly invoked constituent, silicon carbide ( $\mathrm{SiC})$, has been detected in the spectra of many carbon stars (Hackwell, 1972; Treffers \& Cohen, 1974), and is useful because it displays a characteristic spectral feature at about $11 \mu \mathrm{m}$, unlike the carbon which has no diagnostic spectral features in the infrared (IR). The carbon adds to the continuum emission and therefore is detectable, but it cannot be definitively identified as carbon dust. Metallic iron is also abundant as silicon is and is expected to be found in carbon-rich stars. Like carbon, metallic iron does not exhibit diagnostic infrared features. Metallic iron has been largely ignored in the modeling of dust around carbon-rich stars but this is something we are going to ratify and thus it's influence is unknown.

In the work presented here I will studied the effect of different carbon species and iron grains on carbon rich AGB stars' dust shell continuum. But before you can get into the research, we need some proper introduce to our stars. 


\section{Chapter 2}

\section{Stellar Evolution and AGB Stars}

"Oh, gee. I always thought they were balls of gas burning billions of miles away." - Pumbaa (The

Lion King)

\section{$2.1 \quad$ Introduction}

Let's start and take a few moments to understand how we know what we know about stars before going into detail of how stars are formed, live, eventually die and completing the circle of life.

With the exception of our sun, the only direct information we have about the stars comes from the light that we receive from them and their surroundings. When looking at stars in the night's sky, two properties are immediately apparent; how bright the light is and its color. 


\subsubsection{Surface Temperature and Size}

Stars are approximations to blackbodies, so we can relate the star's color with its surface temperature by the use of Wien's Law:

$$
\lambda_{\text {peak }} T=2.898 \cdot 10^{-3} m \cdot K
$$

If we have the temperature and also the brightness, we will then use the StefanBoltzmann equation:

$$
L=A \sigma T^{4}
$$

where $L$ is the luminosity, which is the total power output of the emitting object, $A$ is the surface area of the emitting object, and $\sigma$ is the Stefan-Boltzmann constant

$\left(\sigma \approx 5.6710^{-8} W \cdot m^{-2} \cdot K^{-4}\right)$ and $T$ is the surface temperature of the object. However, the brightness we observe in the night sky differs from the star's true luminosity. This is in part because we only see visible wavelengths, and mainly because the star's light dims with distance. Each star gives off radiation at all wavelengths, and in all directions. The brightness of any light falls off as a function of distance according to the inverse square law:

$$
F=\frac{L}{2 \pi r^{2}}
$$

where $F$ is the flux that we observe, and $\mathrm{r}$ is the distance between us and the light source. Additionally, we need to take in the account the absorption of light that happens in earth's atmosphere as well as in the interstellar medium (ISM; the stuff between us and the star.) For now, we will ignore these and assume that the light 


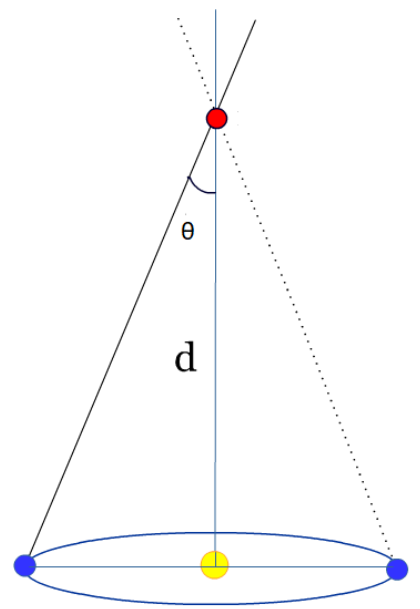

Figure 2.1: Stellar Parallax. The dark sphere represents the sun, the small light sphere is the Earth, and the dashed circle represents the Earth's orbit; $d$ is the average distance between Earth and the star being observed, and $\theta$ is the parallax angle.

absorption factors are negligible. When we speak of flux and luminosity it will be always refer to the flux and luminosity over all wavelengths (unless otherwise stated).

\subsubsection{Distance}

To find the luminosity of a star, we have to determine its distance from us. Measuring distances in space is hard. Consequently distances are not known accurately for many stars. There is one technique that can be used for relatively nearby stars (within about 1000 light years or a few hundred parsecs); that is measuring of the stellar parallax, which is shown in Figure 2.1.

Let's picture an observer on the Earth looking at a nearby star, the apparent position of the star in the sky will change, relative to much more distant stars, as the 
Earth moves around its orbit. If we measure the apparent position of the star at 6 month intervals as seen in the figure above, then the apparent change in the position of the star between those two measurements will be at its maximum. With the help of trigonometry, we can relate this apparent positional change and the distance to the star by:

$$
d=\frac{r_{a . m}}{\sin \theta}
$$

where $r_{a . m}$. is the average distance between the Earth and the Sun, roughly $\sim$ $149.6 \cdot 10^{9} \mathrm{~m}$. For any object outside our solar system, $\theta$ is very small. Our closest star after the sun has $\theta<1$ arcsecond (Carroll \& Ostlie, 1996), Therefore we can use the small angle approximation $(\sin \theta \approx \theta$ (in radians)) to find:

$$
d=\frac{r_{a . m .}}{\theta}
$$

We know $r_{a . m}$., this lets us determine the distance to objects within in our capability of measuring $\theta$. The distance given by $\theta$ of one arcsecond is referred to as a parsec (pc), which is about $3.09 \cdot 10^{16} \mathrm{~m}$ or 3.26 light-year. The Hipparcos satellite has achieved the best measurements of $\theta$ but has only achieved as astrometric accuracy of roughly 0.001 arcseconds, so the furthest distance that can be determined with this method was $1000 \mathrm{pc}$, which is only about $5 \%$ of the diameter of our galaxy (Carroll \& Ostlie, 1996). If one requires measurements beyond this distance then it will involve the use of more complex and indirect techniques that are beyond the scope of this paper. 


\subsubsection{Size}

If the distance is known and we assume that stars approximate spheres, we then can return to Eq. 2.2 and find that:

$$
L=A \sigma T_{\mathrm{eff}}^{4}=4 \pi r^{2} \sigma T_{\mathrm{eff}}^{4} \Rightarrow r=\left(\frac{L}{4 \pi \sigma T_{\mathrm{eff}}^{4}}\right)^{\frac{1}{2}}
$$

where $r$ is the radius of the star, $T_{\text {eff }}$ is the effective surface temperature. For a perfect blackbody $T_{\text {eff }}=T_{\text {color }}$, where $T_{\text {color }}$ is the temperature used in Wien's Law (and thus defines the color of the star). Assuming a perfect blackbody, we can figure out the size of the star.

\subsubsection{Hertzsprung-Russel Diagram}

The Hertzsprung-Russell (HR) diagram plots the stars according to their effective

temperature $\mathrm{T}_{\text {eff }}$ (increasing to the left) and their luminosity (L) on a log-log scale. Luminosity is given relative to the luminosity of the sun, $\mathrm{L}_{\odot}$ seen in Figure 2.2.

Stars will group in certain regions of the HR diagram. These regions correspond to different types of stars and also to different periods of a stars life. From the equation of the luminosity of a star, we can obtain stellar sizes from the HR diagram. The equation of luminosity (Eq. 2.6) is:

$$
r=\left(\frac{L}{4 \pi T_{\mathrm{eff}}^{4}}\right)^{1 / 2}
$$

where, $A$ is the surface area of the star and $r$ is its radius.

The stars in the upper right of diagram are relatively cool but very luminous, so they will have a large radius. However, the stars in the lower left of the diagram are very hot, and have low luminosity, so they must have a small radius. 


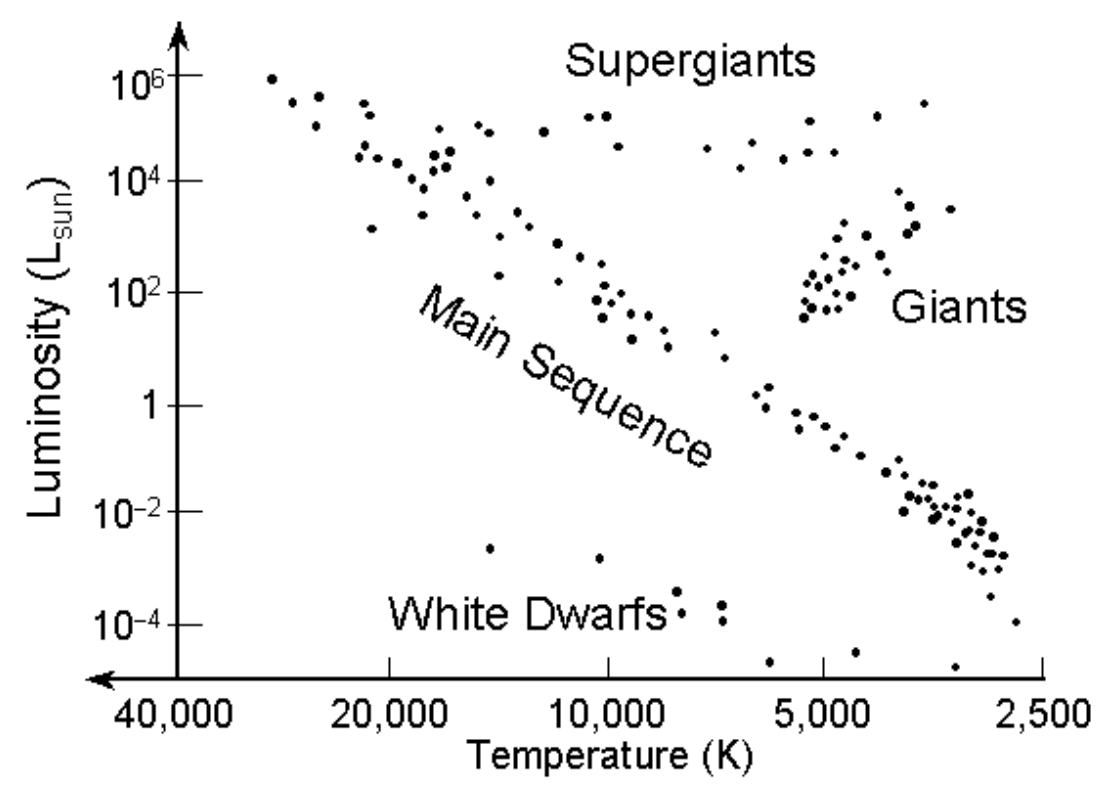

Figure 2.2: Hertzsprung-Russell Diagram showing luminosity vs temperature.

\subsection{Stellar Evolution}

All stars begin the same way, but how far they proceed through different nuclear processes depends on the star's mass. Protostars that are too small $\left(\leq 0.08 \mathrm{M}_{\odot}\right)$ will never start hydrogen fusion ${ }^{1}$; these are brown dwarfs. Very low mass stars $\left(0.08_{-}^{-}\right.$ $\left.0.8 \mathrm{M}_{\odot}\right)$ can achieve hydrogen fusion but will never achieve helium fusion. As the stars go up in mass, will go to the next step until the highest mass star goes all the way through the fusion processes to making iron. There are some stars with masses between $\sim 8-12 \mathrm{M}_{\odot}$ that will fuse carbon but not oxygen, neon, or magnesium. These stars will end up as white dwarfs made of those elements. The reasons for this sequence of fusion happening or not happening will be discussed below.

\footnotetext{
${ }^{1}$ although they do fuse deuterium, a heavy isotope of hydrogen.
} 


\subsubsection{Birth of Stars}

\section{Molecular cloud}

Between stars, space is filled with an extremely tenuous mixture of gas and dust particles, called the interstellar medium. This material originates from past stars and forms new stars, in a cycle of life that has been happening for billions of years.

There will be some regions of space, where this gas and dust can become dense enough to be optically thick (i.e. opaque) at visible wavelength. With molecular clouds, they are optically thick enough for $\mathrm{H}_{2}$ to form and survive; UV radiation is blocked by the opacity of the cloud.

Molecular clouds are held together by gravity. Therefore, if it is in equilibrium then a cloud will satisfy the virial theorem:

$$
2 K+U=0
$$

where $K$ is the internal kinetic energy of the cloud and $U$ is the gravitational potential energy of the cloud. In general, if the kinetic energy is larger than the potential energy, the cloud will expand; whereas if the potential energy is greater, the cloud will collapse. However, under virial theorm, the initial conditions mean that as we convert potential energy to kinetic energy in a collapsing cloud, half the kinetic energy must be radiated away in order to reach equilibrium.

We will examine the boundary condition given by the virial theorem. Lets assume a spherical cloud of uniform density, easier analysis, and gravitational potential energy is approximately given by (Carroll \& Ostlie, 1996): 


$$
U \approx \frac{3 G M_{c}^{2}}{5 R_{c}}
$$

where $R_{c}$ is the radius of the cloud, $M_{c}$ is the mass of the cloud, and $G$ is the gravitational constant. Then, lets assume that the cloud is an ideal gas, we can apply the equipartition theorem to get the kinetic energy of the cloud:

$$
K=\frac{3}{2} N K T
$$

where $N$ is the total number of particles in the molecular cloud, $T$ is the temperature of the cloud, and $k$ is the Boltzmanns constant. We can find $N$ as:

$$
N=\frac{M_{c}}{\mu m_{H}}
$$

where $\mu$ is the mean molecular weight of the cloud relative to hydrogen, and $m_{H}$ is the mass of a hydrogen atom. Then we can rewrite the virial theorem as:

$$
\frac{3 M_{c} k T}{\mu m_{H}}+\frac{3 G M_{c}^{2}}{5 R_{c}}=0 \rightarrow \frac{3 M_{c} k T}{\mu m_{H}}=\frac{3 G M_{c}}{5 R_{c}}
$$

The equality in this equations hold when the cloud is in equilibrium. To have the cloud to collapse, we must replace it by an inequality, the potential energy must be greater than the kinetic energy:

$$
\frac{3 M_{c} k T}{\mu m_{H}}<\frac{3 G M_{c}}{5 R_{c}}
$$

Since we assume a constant initial density, $\rho_{0}, R_{c}$ then can be rewritten as:

$$
\text { Volume }=\frac{M_{c}}{\rho_{0}}
$$




$$
4 \pi R_{c}^{3}=\frac{M_{c}}{\rho_{0}} \rightarrow R_{c}=\left(\frac{3 M_{c}}{4 \pi \rho_{0}}\right)^{1 / 3}
$$

which then allows us to express the virial theorem in terms of mass. By subbing, we obtain that:

$$
\begin{gathered}
\frac{k T}{\mu m_{H}}<\frac{G M_{c}^{2}}{5\left(\frac{3 G M_{c}}{4 \pi \rho_{0}}\right)^{1 / 3}} \\
M^{2 / 3}>\frac{5 k T}{G \mu m_{H}} *\left(\frac{3 G M_{c}}{4 \pi \rho_{0}}\right)^{1 / 3} \\
M_{c}>{\frac{5 k T}{G \mu m_{H}}}^{3 / 2} *\left(\frac{3}{4 \pi \rho_{0}}\right)^{1 / 2}
\end{gathered}
$$

If a cloud has a mass that satisfy the inequality above, it will collapse on itself. This can happen in different ways: one is for the cloud to have a shock strike it, increasing the density. Examining, Eq. 2.18 we see that if the density on right side of the inequality is increased, the required mass for the cloud collapse decreases. Any increase in density will make a cloud in equilibrium to collapse.

Consider what happens to the cloud as it collapses if there are any local regions of higher density, even if it is by a small amount. As the cloud collapses, these regions will then become denser along with the rest of the cloud, the same number of particles but in a smaller volume of space. Then these regions can satisfy the inequality, causing them to locally collapse independently of the rest of the cloud. This will lead to the cloud to fragment into smaller collapsing regions. This demonstrates why stars are expected to form in groups of similar chemical composition, called clusters. At least half of all stars are gravitationally bound to one or more stars (Carroll \& Ostlie, 1996). 


\section{Hydrostatic equilibrium}

Hydrostatic equilibrium is the precise balance of gravity and pressure. In stars, this happens when the energy generated in the core of a star gives rise to a pressure gradient that exactly balances the force of gravity pulling the stars matter inward. Following Carroll \& Ostlie (1996), let's imagine an infinitesimal cylinder of the star's matter with mass $d m$, height $d r$, and base area $A$, located a distance $r$ from the stars middle. Using Newton's Second Law, we can write the net force on the cylinder as:

$$
d m \frac{d^{2} r}{d t^{2}}=F_{\text {gravity }}+F_{\text {top }}+F_{\text {bottom }}
$$

where $\mathrm{F}_{\text {gravity }}$ is the gravitational force on the cylinder, $\mathrm{F}_{\text {top }}$ is the force of the pressure pushing down on the top of the cylinder, and $\mathrm{F}_{\text {bottom }}$ is the force of pressure pushing up on the bottom of the cylinder. The pressure on the top of the cylinder and the pressure on the bottom carry different signs, indicating the direction of the pressure, and differ by the term of $\mathrm{dF}_{\text {pressure }}$ which represents the change in the force of pressure over the change of distance, dr. Then we can write:

$$
F_{\text {top }}=-\left(F_{\text {bottom }}+d F_{\text {pressure }}\right)
$$

By substituting the net force into this equation, we find:

$$
\begin{gathered}
d m \frac{d^{2} r}{d t^{2}}=F_{\text {gravity }}-\left(F_{\text {bottom }}+d F_{\text {pressure }}\right)+F_{\text {bottom }} \\
d m \frac{d^{2} r}{d t^{2}}=F_{\text {gravity }}-d F_{\text {pressure }}
\end{gathered}
$$

The gravitational force on the cylinder is given by:

$$
F_{\text {gravity }}=-G \frac{M_{\text {inside }} d m}{r^{2}}
$$


where $G$ is the gravitation constant, and $M_{\text {inside }}$ is the mass of the star inside radius $r$. The equation for pressure $(P)$ in relation to force is:

$$
P=\frac{F_{\text {pressure }}}{A}
$$

Since the area $A$ of the cylinder does not change with $r$, the differential pressure can be written as:

$$
d P=\frac{F_{\text {pressure }}}{A} \rightarrow d F_{\text {pressure }}=A d P
$$

Then substituting this equation into equation (2.22),

$$
d m \frac{d^{2} r}{d t^{2}}=-G \frac{M_{\text {inside }} \rho A d r}{r^{2}}-A d P
$$

The expression for $d m$ can be found by seeing that the mass in the cylinder is the volume of the cylinder times the density of the material in the cylinder (which can be assumed to be constant), giving:

$$
d m=\rho A d r
$$

Substituting this into equation (2.26), we obtain that:

$$
\begin{gathered}
\rho A d r \frac{d^{2} r}{d t^{2}}=-G \frac{M_{\text {inside }} \rho A d r}{r^{2}}-A d P \\
\rho d r \frac{d^{2} r}{d t^{2}}=-G \frac{M_{\text {inside }} \rho d r}{r^{2}}-\frac{d P}{d r}
\end{gathered}
$$

This is the equation of motion for the cylinder in the radial direction. Since we are looking at situation of when the star is in hydrostatic equilibrium this means the star is not contracting or expanding. The cylinder is motionless and therefore $\frac{d^{2} r}{d t^{2}}$ is 
zero. This leads to the equation to be:

$$
\frac{d P}{d t}=-G \frac{M_{\text {inside }} \rho d r}{r^{2}}
$$

To maintain hydrostatic equilibrium, a pressure gradient given above must occur. The pressure gradient is a result of the temperature gradient that exists due to energy coming from the stars core. Assuming the star is an ideal gas, then the gas pressure generated by the temperature, kinetic energy, of atoms in the star can be written as:

$$
P_{g a s}=\frac{n k T}{V}
$$

where $k$ is the Boltzmanns constant, and $n$ is the number density of particles in volume $V$, at a temperature $T$. Substituting Eq. 2.11, we obtain that:

$$
P_{g a s}=\frac{M_{v} k T}{\mu m_{H} V}
$$

where $M_{v}$ is the mass that is contained in the volume. Under the assumption that the density in $V$ is constant:

$$
P_{g a s}=\frac{\rho k T}{\mu m_{H}}
$$

The gas pressure is not the only pressure present as a result of the temperature. There are a large number of photons generated within in a star; radiation pressure gas cannot be neglected. The pressure generated by a photon gas of temperature $T$ is given by (Huang, 1987):

$$
P_{\text {photons }}=\frac{\pi^{2} k^{4}}{60 c^{3} \hbar^{2}} T^{4}
$$

Combining this equation with Eq. 2.31 gives the total pressure: 


$$
P_{\text {total }}=\frac{\rho \kappa}{\mu m_{H}} T+\frac{\pi^{2} k^{4}}{60 c^{3} \hbar^{2}} T^{4}
$$

Converting this equation so that the variable is radial distance from the center of the star, rather than temperature, is complicated by convection, which can play an important role in the interiors of some stars. For my research, it is sufficient to investigate the basic concepts of hydrostatic equilibrium. Whenever the energy generated by the star is enough to overcome the gravitational force, the star will expand until equilibrium is reached again. Whenever the energy generated is not enough to overcome gravity, the star will contract until the energy output is once again large enough to balance the Eq. 2.32.

\section{Protostars}

As material in a cloud will collapses inwards, the conversion of $U$ to $K$ increases the cloud temperature and density, with the center being hottest and densest. In addition, the cloud spins increasingly fast as it collapses in order to conserve angular momentum. At the same time, the centrifugal force outwards from the rotation axis counters gravity, causing the cloud to flatten and become increasingly disk shaped. Some of the disk material will fall into the star; some of the material will accrete together which will form planets and other objects that remain in the orbit around the star. Most of the rest of the material will be dispersed into space by the radiation pressure as nuclear fusion starts; stellar wind of very rapidly moving charged particles streaming out from the stars surface. 


\subsubsection{Main Sequence}

By definition, a star on the main sequence line is generating energy by fusing hydrogen into helium in its core.

Hydrogen fusion in a main sequence star can happen through two processes that are present in all main sequence stars. Which process dominates energy production in a star depends on the mass of that star. Proton-proton chain provides the majority of the energy for a star below $1.3 \mathrm{M}_{\odot}$. For stars more massive than this then CNO cycle will provide the majority of the energy. We will talk about the CNO cycle a little later.

A star will spend most of its life on the main sequence. This is because hydrogen fusion generates the most energy per atom and also the star contains more hydrogen than any other element. Stars will experience change during this period, since converting hydrogen into helium decreases the mass by $0.7 \%$ but also decreases the number of particles in the core. This happens because when four hydrogens become one helium atom there are fewer total atoms available to contribute in the gas pressure. This will cause the core to shrink as the gas pressure cannot compete with gravity, in turn increasing the density and temperature as $U$ converts $K$. This process increases the fusion reaction rate and thus the energy output enough to return the core to a state of hydrostatic equilibrium. The energy output of the core is always slowly increasing, which makes the star more luminous over time. For an example, the sun has increased in radius by $6 \%$, increased in temperature by $300 \mathrm{~K}$ and increased by luminosity by $30 \%$. 


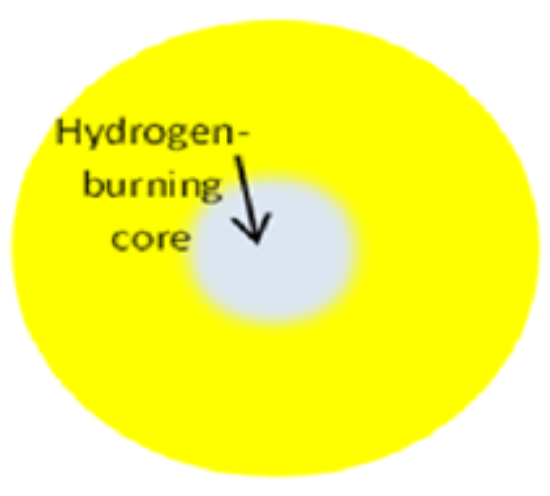

Figure 2.3: Diagram of a main sequence star. The tiny core of the star is fusing hydorgen into helium, and is surrounded by a larger atmosphere that does not participate in the fusion process. This is not to scale.

When combined, these branch reactions produce energy at a rate proportional to roughly $\mathrm{T}^{20}$, which is why this becomes the dominate energy process for higher mass stars. The cores of these stars have a higher temperature than those of lower mass stars. From this we can also see that the metallicity of a star can play a role in which of these processes is dominate. A star with lower metallicity may not have enough carbon atoms to make the $\mathrm{CNO}$ cycle run at the same rate as a star with higher metallicity.

\section{Proton-proton chain}

$$
\begin{gathered}
{ }_{1}^{1} \mathrm{H}+{ }_{1}^{1} \mathrm{H} \rightarrow{ }_{1}^{2} \mathrm{H}+e^{+}+\nu_{e} \\
{ }_{1}^{2} \mathrm{H}+{ }_{1}^{1} \mathrm{H} \rightarrow{ }_{2}^{3} \mathrm{He}+\gamma \\
{ }_{2}^{3} \mathrm{He}+{ }_{2}^{3} \mathrm{He} \rightarrow{ }_{2}^{4} \mathrm{He}+2{ }_{1}^{1} \mathrm{H}
\end{gathered}
$$




$$
\begin{aligned}
&{ }_{2}^{3} \mathrm{He}+{ }_{2}^{4} \mathrm{He} \rightarrow{ }_{4}^{7} \mathrm{Be}+\gamma \\
&{ }_{4}^{7} \mathrm{Be}+e^{-} \rightarrow{ }_{3}^{7} \mathrm{Li}+\nu_{e} \\
&{ }_{3}^{7} \mathrm{Li}+{ }_{1}^{1} \mathrm{H} \rightarrow{ }_{2}^{4} \mathrm{He} \\
&{ }_{4}^{7} \mathrm{Be}+{ }_{1}^{1} \mathrm{H} \rightarrow{ }_{5}^{8} \mathrm{~B}+\gamma \\
&{ }_{5}^{8} \mathrm{~B} \rightarrow{ }_{5}^{8} \mathrm{~B}+e^{+}+\nu_{e} \\
&{ }_{5}^{8} \mathrm{~B} \rightarrow 2_{2}^{4} \mathrm{He}
\end{aligned}
$$

When combined, these branch reactions create energy at a rate proportional to $\sim T^{4}$. For the remainder of this chapter, $T$ refers the core temperature and is relevant to nuclear processes.

\section{CNO cycle}

The CNO cycle converts hydrogen into helium. The CNO cycle uses carbon atoms as catalysts for the reaction, but they are not consumed (Carroll \& Ostlie, 1996).

$$
\begin{aligned}
{ }_{6}^{12} \mathrm{C}+{ }_{1}^{1} \mathrm{H} & \rightarrow{ }_{7}^{13} \mathrm{~N}+\gamma \\
& { }_{7}^{13} \mathrm{~N} \rightarrow{ }_{6}^{13} \mathrm{C}+e^{+}+\nu_{e} \\
{ }_{6}^{13} \mathrm{C}+{ }_{1}^{1} \mathrm{H} & \rightarrow{ }_{7}^{14} \mathrm{~N}+\gamma \\
{ }_{7}^{14} \mathrm{~N}+{ }_{1}^{1} \mathrm{H} & \rightarrow{ }_{8}^{15} \mathrm{O}+\gamma \\
& { }_{8}^{15} \mathrm{O} \rightarrow{ }_{7}^{15} \mathrm{~N}+e^{+}+\nu_{e} \\
{ }_{7}^{15} \mathrm{~N}+{ }_{1}^{1} \mathrm{H} & \rightarrow{ }_{6}^{12} \mathrm{C}+{ }_{2}^{4} \mathrm{He}
\end{aligned}
$$




$$
\begin{aligned}
& \quad{ }_{7}^{15} \mathrm{~N}+{ }_{1}^{1} \mathrm{H} \rightarrow{ }_{8}^{16} \mathrm{O}+\gamma \\
& { }_{8}^{16} \mathrm{O}+{ }_{1}^{1} \mathrm{H} \rightarrow{ }_{9}^{17} \mathrm{~F}+\gamma \\
& \quad{ }_{9}^{17} \mathrm{~F} \rightarrow{ }_{8}^{17} \mathrm{O}+e^{+}+\nu_{e} \\
& { }_{8}^{17} \mathrm{O}+{ }_{1}^{1} \mathrm{H} \rightarrow{ }_{7}^{14} \mathrm{~N}+{ }_{2}^{4} \mathrm{He}
\end{aligned}
$$

\subsubsection{Red Giants}

Once a star has consumed all the hydrogen available in the core, it is no longer able to generate energy through hydrogen fusion. This will cause the core to collapse under gravity as the gas and radiation pressures both drop. This collapse increases both temperature and density in the stellar core as potential energy is converted to kinetic energy. The material around the core will also collapse inwards increasing its temperature and density. This shell of hydrogen around the core becomes dense and hot enough to fuse hydrogen into helium. The reactions happen at much greater rate due to the continuous increase of temperature generated by the collapse of the core. Several orders of magnitude more energy is created by this shell of fusion, this causes the envelope of the star outside this fusion shell to expand until it reaches hydrostatic equilibrium. When a star is in this state, it is called a red giant.

The expansion of the stellar envelope cause the star to have a lower surface temperature than it did when it was a main sequence star. This is because even though, the star is generating more energy in its interior, and the stars surface layers are much further away. Red giants more luminous than main sequence stars of the same mass. 
The precise details of how stars evolve are strongly dependent on the initial masses of those stars which we will discuss later.

\section{Degenerate matter}

Since electrons are fermions, the will fall victim to the Pauli Exclusion Principle and there can only be one electron in each quantum state.

Lets consider a collection of $N$ non-interacting electrons bound inside a cube with sides of length $L$, where the potential is zero inside of the cube and infinite outside. The allowed energies of each electron are as follows:

$$
E_{n 1, n 2, n 3}=\frac{\hbar^{2} \pi^{2}}{2 m_{e} L^{2}}\left(n_{x}^{2}+n_{y}^{2}+n_{z}^{2}\right)
$$

Where $n_{x}=1,2,3, n_{y}=1,2,3 .$. , and $n_{z}=1,2,3$ are the quantum numbers, $m_{e}$ is the electron mass. We will define the vector $\vec{w}$ as:

$$
\vec{w}=n_{x} \vec{x}+n_{z} \vec{z}+n_{z} \vec{z}
$$

Imagine a three-dimensional vector space with $\vec{w}$ as its basis, and then each point $\left(w_{x}, w_{y}, w_{z}\right)$ in the space corresponds to the allowed energy.

$$
E_{\vec{w}}=\frac{\hbar^{2} \pi^{2}\|\vec{w}\|}{2 m_{e} L^{2}}
$$

An energy level, $E_{\max }$, corresponds to the situation where every quantum state with energy below $E_{\max }$ is filled with an electron and no electrons have energy above this. The energy corresponds to the vector $\vec{w}_{\max }$ in the $\vec{w}$ space. When $\mathrm{N}$ is large, then the number of allowed states with energies below $E_{\text {max }}$ can be approximated by 
the fraction of the volume of a sphere of radius $\left\|\vec{w}_{\max }\right\|$ which will lie in the $\vec{w}_{\max }$ space. Also, there will be a factor of two, since each energy state can contain two electrons one for each value of the spin. The total number of states below $E_{\max }$ is given by:

$$
N_{\text {states }}=2 * \frac{4 \pi}{24}\left\|\vec{w}_{\max }\right\|^{3}
$$

If we define $E_{\max }$ as the ground state of this system, by definition the numbered of filled states is given by the number of electrons, $N$, and then $N_{\text {states }}=N$. This means we can write $N$ as:

$$
N=2 * \frac{4 \pi}{24}\left\|\vec{w}_{\max }\right\|^{3} \Rightarrow\left\|\vec{w}_{\max }\right\|=\left(\frac{3 N}{\pi}\right)^{1 / 3}
$$

If we substitute this equation into (2.37):

$$
E_{\text {max }}=\frac{\hbar^{2} \pi^{2}\|\vec{w}\|}{2 m_{e} L^{2}}=\frac{\hbar^{2}\|\vec{w}\|}{2 m_{e} L^{2}}(3 N \pi)^{2 / 3}
$$

If we substitute the volume of the cube for the length, $V=L^{3} \rightarrow L^{2}=V^{2 / 3}$, we get:

$$
E_{\max }=\frac{\hbar^{2}}{2 m_{e}} \cdot\left(\frac{3 N \pi^{2}}{V}\right)^{\frac{2}{3}}
$$

This equation is known as the Fermi energy, $\mathrm{E}_{f}$. The Fermi energy marks the division between the occupied and unoccupied states of the system. If the volume of the system is to decrease, then some of the electrons must have enough energy to transition to 
states above the Fermi energy, if the electrons cannot obtain this energy then the volume cannot compress.

To calculate the pressure, consider the work done by the pressure if it expands the cube by $d V$ :

$$
d w=P d V
$$

The work done, $d W$, is equal to the change in the total energy of the system, $\mathrm{dE}_{\text {total }}$. To calculate the total energy of the system, consider that the Fermi energy gives us the energy of the highest filed state of the system of $N$ electrons. This is true for any value of $N$, the Fermi energy of a system of n electrons give the energy of the two nth electrons. To obtain the total energy of the system by integrating the Fermi energy over all N:

$$
\begin{aligned}
E_{\text {total }} & =\int_{0}^{N} E_{f}(n) d n \\
& =\frac{\hbar^{2}}{2 m_{e}}\left(\frac{3 \pi^{2}}{V}\right)^{2 / 3} \int_{0}^{N} n^{2 / 3} d n \\
& =\frac{3}{5} N \frac{\hbar^{2}}{2 m_{e}}-\frac{2}{3} V^{-5 / 3} \\
& =-\frac{2 N E_{F}}{3 V} \\
& =\frac{\hbar^{2}}{2 m_{e}}\left(\frac{3 N \pi^{2}}{V}\right)^{2 / 3} * \frac{3}{5} N \\
& =\frac{\hbar^{2}}{2 m_{e}}\left(\frac{3 \pi^{2}}{V}\right)^{2 / 3} * \frac{3}{5} N^{5 / 3}
\end{aligned}
$$

To use the equation (2.40) in the work done equation, we have to calculate the derivative of this with the respect to $V$ : 


$$
\begin{aligned}
\frac{d E_{\text {total }}}{d V} & =\frac{3}{5} N * \frac{d E_{F}}{d V} \\
& =\frac{3}{5} N E_{F}
\end{aligned}
$$

The negative sign is to be expected since as the volume increase the total energy decrease. Now we can obtain the equation for pressure from equation (2.40):

$$
P=\frac{d W}{d V}=-\frac{d E_{t o t a l}}{d V}=\frac{2 N E_{F}}{3 V}
$$

Degeneracy can begin as stars collapse under gravity and this can effects the evolution. Low mass stars, become red giants when their core hydrogen is depleted, but their cores become degenerate and prevent further collapse. Energy from shell hydrogen fusion can ignite the degenerate helium core, which will give rise to a helium flash. These stars then develop a degenerate carbon-oxygen core on the asymptotic giant branch. They never produce enough energy to overcome the core degeneracy. Intermediate mass stars go through a similar evolutionary stage to low mass stars, but after a short period on the red giant branch phase they ignite their core helium without a flash and spend an extended period in the red clump before forming a degenerate carbon-oxygen core. More detail on the importance on degeneracy is given in the next section.

\section{Evolution as a red giant}

After the main sequence, hydrogen fusions ceases and so the core loses energy and the pressure in the core drops, consequently the collapses in on itself as the pressure no longer balances gravity. Eventually the core becomes so compressed that it becomes 


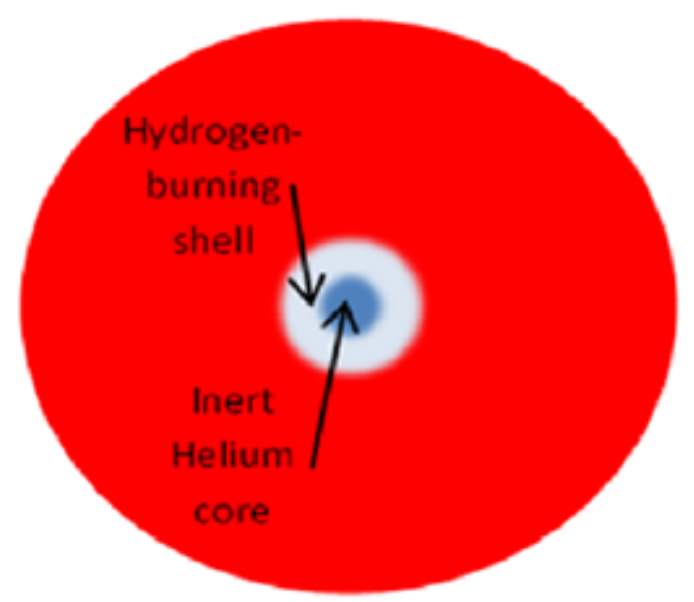

Figure 2.4: Diagram of a red gaint. The inert core of helium is surrounded by a shell where hydorgen is being fused into helium, which is surrounded by a much larger inert envelope. This is not to scale.

degenerate. This happens in low mass stars $\left(0.08-2.5 \mathrm{M}_{\odot}\right)$. Degeneracy pressure is independent of the temperature; the core can freely increase in temperature without expanding and cooling as energy is generated by hydrogen fusion in the shell around the core (discussed above and shown in Figure 2.4).

For stars above $0.8 \mathrm{M}_{\odot}$, eventually the core will become hot enough for helium to being fusing into carbon; we will talk about this process later. Degenerate matter is a good conductor of heat. Once helium fusion begins it will spread rapidly to the entire core. Since the core is degenerate, the rapid temperature increase does not expand and cool the core of the star, and also the rate of fusion reactions increases. This makes the temperature increase, which increases the reaction rate, causing the core to release a massive pulse of energy called the helium flash; this event happens 
over a period of only a few seconds (Freedman \& Kaufmann, 2005). This provides enough energy to break the degeneracy; many electrons can transition above the Fermi energy. This means that the pressure due to temperature dominates, forcing the core to rapidly expand and stabilize the rate of helium fusion.

\section{Stars below $0.8 \mathrm{M}_{\odot}$}

For stars with a mass below $0.8 \mathrm{M}_{\odot}$ will never fuse helium and die as a helium white dwarf.

\section{Stars between 0.8 and $2.5 \mathrm{M}_{\odot}$}

For stars between 0.8 and $2.5 \mathrm{M}_{\odot}$, shrinkage of the core is limited by electron degeneracy. The core volume will have decreased to the point where the matter is degenerate matter. The pressure due to quantum mechanical interactions between the electrons in the core dictates the pressure.

\section{Stars between 2.5 and $8 \mathrm{M}_{\odot}$}

Stars between 2.5 and $8 \mathrm{M}_{\odot}$ evolve in a similar way to lower mass stars, but their more massive cores reach temperatures high enough to fuse helium before their cores become dense enough to be degenerate. Consequently, helium fusion begins much more gradually in their cores, and they transition into the next phase of evolution without a helium flash. For these stars, degeneracy kicks in the next phase of evolution, whereas stars more massive than $8 \mathrm{M}_{\odot}$ have hot enough cores to avoid the problems of electron degeneracy. 


\subsubsection{Horizontal Branch}

Once core helium fusion is initiated, the star will transition to the horizontal branch on the HR diagram, see Figure 2.2. These stars are similar to main sequence stars, because they are again producing energy in the core. In addition to the core helium fusion, the hydrogen burning shell is active; however the rate of hydrogen fusion reactions greatly decreases due to the core expansion, which expands the hydrogen fusion shell. This makes the total energy output of the star decrease and therefore, the luminosity decreases. The outer envelope shrinks as the pressure decreases, which increases the star surface temperature.

\section{Triple alpha process}

During the horizontal branch phase of a star's evolution, helium is the fuel, and the ash is carbon. This nucleosynthetic process is know as the Triple-Alpha process. It converts three helium nuclei into one carbon nucleus:

$$
\begin{aligned}
& { }_{2}^{4} \mathrm{He}+{ }_{2}^{4} \mathrm{He} \rightleftharpoons{ }_{4}^{8} \mathrm{Be} \\
& { }_{4}^{8} \mathrm{Be}+{ }_{2}^{4} \mathrm{He} \rightarrow{ }_{6}^{12} \mathrm{C}+\gamma
\end{aligned}
$$

This reaction has a rate proportional to $\mathrm{T}^{4}$ (Carroll \& Ostlie, 1996).

At high temperatures there are other, minor fusions reactions happening in the core. For stars with masses that we are interested in, the most important reactions is when the carbon atom generated in step two of the tripe-alpha process captures another helium atom, creating oxygen: 


$$
{ }_{6}^{12} \mathrm{C}+{ }_{2}^{4} \mathrm{H} \rightarrow{ }_{6}^{16} \mathrm{O}+\gamma
$$

\section{End of the Horizontal Branch}

The star will eventually run out of helium in its core. This happens faster than when the star ran out of hydrogen, because (1) there were fewer helium atoms available than there were hydrogen atoms; and (2) each reaction generates less energy and the star is brighter. The reactions rates have to be higher to create enough energy to maintain hydrostatic equilibrium. The end of helium fusion causes the stellar core to collapse again until it becomes degenerate, this happens for all stars with mass between $0.8-8 \mathrm{M}_{\odot}$. As with the first red giant phase, the collapse of the core drags the unused helium, created by the hydrogen burning shell, along with it. Again, this shell of material heats until it can fuse the helium atoms through the triple-alpha process.

\subsubsection{Asymptotic Giant Branch}

Asymptotic giant branch or AGB stars consist of a degenerate core of carbon, surrounded by a shell of helium being fused into carbon, surrounded by a shell fusing hydrogen into helium, surround by an inert stellar envelope.

Energy production inside an AGB star varies as the helium and hydrogen fusion shells are not usually active simultaneously. There are two reasons for the switching of nuclear fusion processes: 1) The helium burning shell is much thinner than the hydrogen burning shell; the star can run out of helium fuel; and 2) helium burning is temperature dependent, causing the process to shut down if the high temperature 
is not maintained. When fusion switches off, the remaining helium is compressed by gravity. During this time, hydrogen fusion starts up because it also collapses under gravity and heats up. The hydrogen shell is the only region of the star that is producing energy. The helium generated through the hydrogen fusion falls into the region that was formerly helium burning, increasing its temperature and density. The density can increase so much that this region, can become degenerate, though still dominated by the thermal pressure. Eventually, this leads to a kind of low-order helium flash, when the region becomes hot enough to fuse helium again. This onset of helium-fusion causes the overlying layers to expand, which cools the hydrogen burning shell and can even cause it to stop fusion. The luminosity of the star increases greatly while the helium burning shell is active. This period is relatively short, however, because the helium shell expansion causes cooling below the threshold for fusion. Consequently the helium shell will reduce its energy output and finally deactivate. Then the outer layers of the star contract, which will heat the hydrogen shell and reactivate it. This process will continue through the rest of the stars AGB life and is known as the thermally pulsing AGB phase.

\section{Pulsation}

Thermal pulses that result from the fusion reactions periodic activation and deactivation, have timescales of the order of 10,000 to 100,000 years. AGB stars also pulsate on much shorter timescale, of the order of a year (60-1,000 days). In order to understand the pulsation mechanism, we must the understand the composition of stars envelope. The envelope consists primarily hydrogen ( $75 \%$ by mass), and he- 


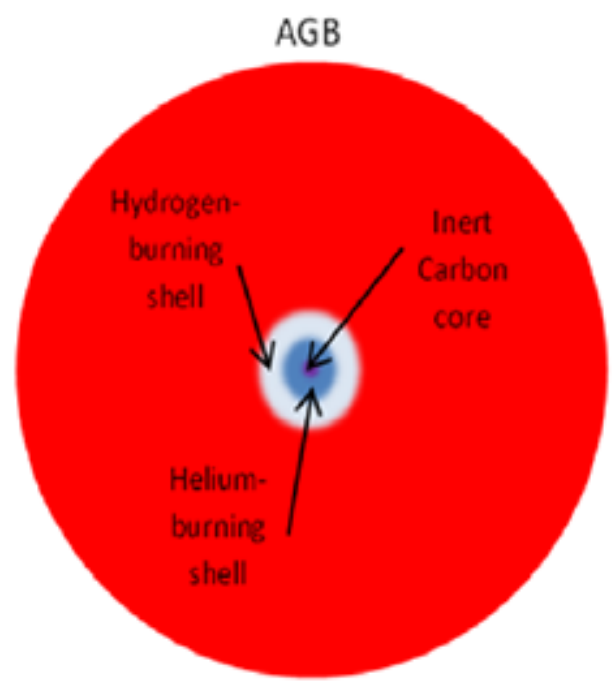

Figure 2.5: Diagram of an AGB star. The inert core of carbon is surrounded by a shell where helium is being fused into carbon, which is surrouneded by a shell where hydrogen is being fused into helium. All of this is surrounded by a much larger inert envelope. 
lium. Other elements typically constitute less than $1 \%$ by mass, and most of these were present during the stars formation. Let's first look at hydrogen atoms, which have a single electron. At high enough temperatures the electron can receive enough energy to escape the atom, ionizing it. The star's temperature increases with depth; we can picture a radius below which all hydrogen atoms have lost their electron becoming fully ionized. These two radii do not coincide; between these two radii is a layer where there is a partial ionization. The relatively high concentration of helium atoms can also follow a similar pattern, but now there are two electrons that can be removed from the atom. This zone is called the partial ionization zone. A small change in temperature in this region can cause a large change in the ionization state.

We will see later that the opacity of a material determines how well it can absorb the light passing through it. The relationship between the opacity, the density and the temperature for most stars envelope is given by Kramers Law (Carroll \& Ostlie, 1996):

$$
\chi \propto \frac{\rho}{T^{3.5}}
$$

where $\chi$ is the opacity of the material, $\rho$ is the density and $T$ is the temperature. Kramers Law holds for the partial ionization zone.

If we were to compress the star's material, we would increase its density, which tends increase the opacity. However, if we apply Kramer's Law (2.44), the temperature also increases as it is compressed, the total opacity will decrease. Imagine a zone being compressed, the density increases, increasing the opacity. In this case, a large fraction of the energy from the compression that would normally go towards heat 
and expanding the zone is used instead to ionize the atoms/ions. Increasing opacity makes the zone absorb more energy from the interior of the star, which will expand outwards, pushing the material with it.

As this zone expands it will cool and decrease in density. While we might expect the star to attain hydrostatic equilibrium through this process, it cannot because as the gas in the zone cools and the electrons will start to recombine with the ionized atoms. This will release energy, which will heat the zone and change the opacity again because it is a different composition. The heating due to the recombination counteracts the temperature decrease due to the expansion, making the density term in Kramers Law to be dominate, and decrease in density which leads to a decrease in opacity. The zone is absorbing less energy from the interior of the star, and will fall back towards the center, leading to compression, and the cycle will start over again.

Elements besides hydrogen can have partial ionization zones. These partial ionization zones are more complex since hydrogen only has one electron; heavier elements have more and therefore can have more than one partial ionization zone. Helium dominates this pulsation process for AGB stars. The location of the ionization zones will depend on the temperature of the star. If the star is relativity hot, the partial ionization is near the surface. If the star is relativity cool, the partial ionization zone will be deep inside the star. The location of the zones plays an important role because if it lies too deep within a star, the envelope above the zone will be too massive for the zone to push effectively. If the zone lies to close to the surface, then it does not have enough material to push to create a significant pulsation. 


\section{Chemistry of AGB stars}

Typically the surfaces of stars, and their atmospheres, reflect the original composition of the star and any non-primordial element (those more massive than helium) were produced in a previous generation of stars. However, some stars can enrich their surface regions with new elements born within the star itself. We know that the triple-alpha process converts helium into carbon, in the helium burning shell. When the helium burning shell is active, it will release such a large amount of energy that it cannot all be radiated away due to opacity of the overlying layers of material. Consequently, powerful convection currents develop that can mix the elements generated by the helium burning shell up into the region between the fusing shells and into the hydrogen burning shell, and finally into the envelope and surface of the star.

Since the convection currents dip down into the helium and hydrogen shells, newly formed elements are mixed up to the surface of the star. These new elements include the carbon generated in the triple-alpha processes as well as the products of neutron capture. The s-process, also known as slow neutron capture process, occurs at relatively low neutron density and intermediate temperature conditions. Under these conditions heavier nuclei are made by neutron capture, increasing the mass of the nucleus by one. A neutron in the nucleus decays by $\beta$-decay to a proton, making a nucleus of higher atomic number. The rate of neutron capture by atomic nuclei is slow relative to the rate of radioactive $\beta$-decay. If $\beta$-decay can occur at all, it almost always occurs before another neutron can be captured. The s-process makes approximately half of the isotopes of the elements heavier than iron, and plays an 
important in the galactic chemical evolution. The more rapid, r-process, differ from the s-process by its faster rate of neutron capture of more than one neutron before $\beta$ -decay takes place.

A star which forms in our galaxy has an initial ratio of carbon to oxygen $(\mathrm{C} / \mathrm{O})$ ratio in its envelope, of roughly 0.4 (Thompson et al., 2006). Stars that go through an AGB phase (initial mass of 0.8-8 $\mathrm{M}_{\odot}$ ) all manufacture the dredge-up carbon atoms. Since carbon monoxide $(\mathrm{CO})$ is very stable at the temperatures associated with the surfaces of AGB stars, this essentially locks in the element with lower abundance, removing it from further reactions. The subsequent chemistry of the dust shell is dominated by whichever one is the dominant element abundant. If the $\mathrm{C} / \mathrm{O}$ rises above unity, these stars are known as carbon stars (C-stars).

The mass determines whether an AGB star will become carbon-rich. This mass dependence can be better understood by looking what happens in the interior of stars outside of the 2 to $4 \mathrm{M}_{\odot}$. With stars below $2 \mathrm{M}_{\odot}$, two processes interact to prevent the $\mathrm{C} / \mathrm{O}$ ratio from increasing sufficiently: (1) these stars make less carbon in the helium burning shell and do it more slowly. (2) the convection currents in these stars are not strong enough and do not extend as deeply than those of more massive stars, which means less material is dredged up. Consequently, although the surface does get enriched with carbon atoms and other elements, the total number of carbon atoms dredge up is not sufficient to raise $\mathrm{C} / \mathrm{O}$ to greater than unity. Conversely, stars that have masses greater than $4 \mathrm{M}_{\odot}$, are very effective at both creating $\mathrm{C}$-atoms and dredging up material, but the carbon atoms formed in the helium-burning shell tend to be destroyed in the hydrogen-burning shell. The CNO cycle in the hydrogen- 
burning shell tends to convert carbon to nitrogen. The steps of the CNO cycle take place rapidly until ${ }_{7}^{14} \mathrm{~N}$ is created. Nitrogen-14 is a stable isotope with a small capture cross section, which means it takes a long time for a nitrogen atom to capture another hydrogen atom and continue the rest of the cycle. When carbon passes through the hydrogen burning shell, if the reaction rate of the initial parts of the cycle is rapid enough much of the carbon created will be consumed and locked into ${ }_{7}^{14} \mathrm{~N}$. Whereas the $\mathrm{CNO}$ cycle has time to go to completion in the main-sequence stars, the dredge-up process means that the nitrogen is often removed from the hydrogen burning shell prior to interactions with another hydrogen atom. Thus, stars above the $4 \mathrm{M}_{\odot}$ do not become carbon-rich at their surfaces.

The range of stellar masses that can become carbon-rich depends strongly on metallicity of the stars. Imagine two equal-mass stars at the same stage of evolution in the AGB phase but with different metallicities. These stars should make the same quantity of carbon atoms, but the effect of those C-atoms is different. At lower metallicity there will be less oxygen atoms as a function of mass of the star, and thus $\mathrm{C} / \mathrm{O}$ reaches unity after less carbon atoms have been produced. A star with higher metallicity will have to dredge up more carbon atoms than the lower metallicity star for the same change in the surficial $\mathrm{C} / \mathrm{O}$ ratio. This makes stars with lower metallicity become carbon rich more easily, expanding the range of stellar masses that become carbon rich. Metal-rich stars of a given mass have slightly larger radii and lower effective temperatures than stars of the same mass but lower metallicity. This means that the metal-rich star will have lower gravities and lower escape velocities than lower metallicity stars. With using Kramer Law, Eq. (2.44), the higher the 
effective temperature of the star is, the lower the opacity is. Convection will become the dominant energy transport mechanism when opacity is high and temperatures are low. For instance, the Large Magellanic Cloud ${ }^{2}$ has approximately half the metallicity of our galaxy, and those the stars that become carbon rich range for 1-6 $\mathrm{M}_{\odot}$. Similarly, the Small Magellanic cloud only as $10 \%$ of solar metallicity and is consequently dominated by carbon-rich AGB stars (Matsuura et al. 2005).

\section{Dust shell formation}

During their AGB phase, stars are much larger than during their main-sequence phase, but still have essentially the same mass. This gives them low surface gravity, so that material at the surface can escape more easily. Every time a star pulsates, this surface material is accelerated outwards and some will achieve escape velocity. We know from the stellar spectral classification sequence that AGB stars have molecules low in their atmospheres. These molecules along with atomic gas, will move away from the star, cooling the gas and molecules. As the temperature decreases, the outflowing gas first forms more molecules and then condenses into dust grains. Once the material condenses, it is able to absorb all wavelengths of light emitted by the star and is thus more susceptible to radiation pressure. Radiation pressure accelerates the dust outwards (Lamers \& Cassinello, 1999). Dust particles collide with gas atoms imparting momentum to the atoms and thus dragging the circumstellar gas along with the dust grains. As a result of this outward push/drag of dust/gas, the gas

\footnotetext{
${ }^{2}$ The Large Magellanic Cloud is half solar metallicity. The metallicity in the galaxy varies with distance from the center and height above the galactic plain.
} 
pressure near the star will decrease, and more material flows off the stars surface. Thus the outflowing material is dust driven. The rates at which stars lose mass are expected to be strongly correlated with dust formation.

The nature of the dust that forms around the AGB star will depend on the chemistry of the star's atmosphere, which, as discussed above, can largely be described as either oxygen-dominated or carbon-dominated.

Determining what types of dust form is complex. Dust condensation depends on both the pressure-temperature space and the composition of the gas from which it is forming. The $\mathrm{C} / \mathrm{O}$ ratio, nitrogen abundance and abundance of s-process elements all depend on the dredge up by the star. The abundances of other elements depend on metallicity. The pressure-temperature regime around the star depends on the temperature of the star as well as the mass-loss rate and the outflow velocity.

For stars with $\mathrm{C} / \mathrm{O}>1$ (carbon stars), the dust will be dominated by carbon grains, which will form as amorphous or graphitic particles. However, solid carbon grains are difficult to probe through observations because they do not have any diagnostic spectral features in the infrared. The next most important grain to form around the carbon stars is silicon carbide $(\mathrm{SiC})$ which has diagnostic spectral feature in the infrared, at roughly $11 \mu \mathrm{m}$.

Dust shells are expected to have heterogeneities and anisotropies in their density structure as a result of pulsation-driven dust formation and ensuing hydrodynamic turbulent effects (Woitke 2006). Dust formation models suggest that carbon star mass-loss should be modulated on multiple timescales, especially that of the pulsation cycle. Also, pulsations give rise to shock in the outflow gas, which are predicted to 
have a strong effect on local conditions (Cherchneff 2006) and therefore could affect the type of dust that forms.

The spatial scale of heterogeneities which occur from turbulence and shock is expected to be small. The small spatial-scale structure can present observational challenges. One example is that if the local density increases it is expected to result from the pulsation-driven mass loss. The timescale for pulsation is much shorter than the timescale over which the star has been losing mass. Spectroscopic studies include dust produced by several to many consecutive pulsation cycles. The inhomogeneities in the density structure are expected to be eliminated over time by the subsequent hydrodynamic interactions (Villaver al. 2002 a,b).

\subsection{Death of a Star}

As mass flows out and the star gets more luminous, the combination of lower gravity and higher radiation pressure inside the star gives rise to a big star. The mass loss increases for three reasons: (1) the dust-driven mass-loss mechanism has a positive feedback process, which means that more mass loss leads to even more mass loss; (2) as the star gets bigger, the surface gravity decreases, making it easier to eject material; and (3) the luminosity of AGB stars increases over time, making radiation pressure more effective at driving dust outwards. The increased mass-loss means more dust can be produced. Thus, the coupling between dust and radiation pressure also increases. Eventually, the star has become so big and lost so much material that the density in it's outer envelope is extremely low and the pressure in the gas envelope 
can no longer support it against gravity. This leads to the stellar envelope shrinking, collapsing under gravity, causing it to get hotter. Since it shrinks and gets hotter, the luminosity stays about the same. This is called the post AGB or pre-planetary nebula (PPN) phase.

Earlier in a star's life, it overcame degeneracy in its core to start helium fusion. For star's with masses $<8 \mathrm{M}_{\odot}$ the carbon becomes degenerate. This time, the star cannot overcome the degeneracy pressure and ignite another round of nucleosynthesis/fusion. The core will never hot enough.

\subsubsection{Planetary Nebulae}

As the outer layers collapse onto the degenerate carbopn core, the star has shed nearly its entire envelope. The collapsing gas is converting $U$ to $K$ again and heats up from $\sim 3000 \mathrm{~K}$ to around $30,000 \mathrm{~K}$ during the post-AGB or PPN phase. Once it reaches $\sim 30,000 \mathrm{~K}$, the stars temperature is sufficient to ionize the material ejected by the star during the AGB phase. This causes the gas in the circumstellar shell to fluoresce, i.e. generate visible light. The remnant star continues to get hotter and smaller, up to about $200,000 \mathrm{~K}$.

At the same time, the collapse stellar envelope devlops a fast stellar wind, which has a much higher velocity than the earlier material thrown out by the star, but containg much less mass. The fast wind sweeps up the previously ejected material, compressing it and forming much of the material into a shell-like relatively thin layer around the star; for reasons not entirely understood, however, this shell of material 
around the star can form complicated structures (Kwok 2000). This is also beyond the scope of my study. The planetary nebula phase of a star's life is very short, a few tens of thousands of years at the maximum. This is because the nebula can only be seen when the gas and dust ejected during the AGB phase is sufficient dense and close the remnant core. Over time, the nebula drifts outwards and disperses.

\subsubsection{White dwarf}

Once the core of the star get up about $200,000 \mathrm{~K}$, the envelop has no more $U$ to convert to $K$ and the newly dormant remnant star begins to cool. The remaining extremely hot core is called a white dwarf. Since a hot object emits energy at a rate much higher than a cooler one, the white dwarf initially cools relatively quickly, losing the ability to emit enough ultraviolet photons to ionize the any remaining material around it.

The white dwarf is very small, and has a low luminosity for its temperature and because of this, the star can remain hot for a very long time. White dwarfs that are older than our galaxy have surfaces temperatures of thousands of kelvins (Fontaine et al. 2001).

\subsubsection{Conclusion of Stellar Evolution}

Through this chapter we have learned how stars form from interstellar gas and dust; how they subsequent live and evolve and finally end during the star's death throes.

LIM stars throw off large quantities their mass (40-80\% of their initial masses), 
which spreads out into space and eventually become incorporated into molecular clouds and new generations of stars can be formed. The life, evolution and death of these stars help create everything that is around us. 


\section{Chapter 3}

\section{Optical Properties}

"The limit does not exist. The limit does not exist!" - Cady Heron (Mean Girls)

\subsection{Introduction}

Most of the knowledge that we have about dust in space comes from the interactions between light and that dust. It is best if we examine some of the basic concepts and principles behind these interactions, such as how light propagates through dust and determining optical properties of solids. 


\subsection{Classical dispersion analysis}

To understand how light interacts with a dust grain; we first understand how optical properties of a material are determined through laboratory measurements. One way is classical dispersion analysis. Remember from Maxwells equations, we can write that:

$$
D=\epsilon_{0} E+P
$$

Where $D$ is the electric displacement, $\epsilon_{0}$ is the electric permittivity of free space, and $P$ is the polarization. Assume that we are dealing with a linear, homogeneous and isotropic material under the influence of an electric field $E$, we can write:

$$
P=\epsilon_{0} \chi E
$$

Where $\chi$ is the electric susceptibility of the material. By combining these two equations, we find:

$$
\begin{aligned}
D & =\epsilon_{0} E+\epsilon_{0} \chi E \\
& =\epsilon_{0}(1+\chi) E \\
& =\epsilon E
\end{aligned}
$$

Where $\epsilon=\epsilon_{0}(1+\chi)$ and is called the relative permittivity of the material or also called the dielectric function. The dielectric function is a function of the frequency of the electric field impinging on the material, and in general it is complex valued. With the very basics covered, we now can look at the classical dispersion method. We will 
start talking about this method by creating a crude, but powerful model of electrons in matter called the Lorentz model.

\subsubsection{Lorentz Model}

The Lorentz model is basic. Picture an electron of mass $\mathrm{m}$ and charge $q$ connected to a spring with a spring constant $k$, then assume at the other end of the spring is fixed. The force on the electron from the spring is given by $F=-k x$, where $x$ is the displacement of the electron. The equation of the electron is then given by:

$$
m\left(d x^{2}\right) /\left(d^{2} t\right)=-k x
$$

This has the following solutions, neglecting the phase:

$$
x=A \sin \left(2 \pi v_{0} t\right)
$$

Where $A$ is the amplitude of the motion, $t$ is the time and the natural frequency of the motion is given as:

$$
v_{0}=\frac{1}{2 \pi} \sqrt{\frac{k}{m}}
$$

Now we will make this model more complex by adding a damping force $b$ which resists the movement of the electrons, kind of like a frictional force. This is represented by:

$$
F_{d}=-b \frac{d x}{d t}
$$


We will also now let the electric field to influence the electron and assume that $E$ is harmonic with frequency, $\nu$. From the Lorentz Force Law, we are able to write the force on the electron due to $E$ as:

$$
F_{E}=q E
$$

There is no magnetic field in our model. Now to derive the new equation of motion, we have to factor in both this force and the damping force, given by:

$$
m \frac{d x^{2}}{d^{2} t}=-k x-b \frac{d x}{d t}+q E
$$

To help us solve this equation, lets rearrange the terms in the following way:

$$
\frac{d x^{2}}{d^{2} t}+\frac{b}{m} \frac{d x}{d t}+\frac{k}{m} x=\frac{q E}{m}
$$

We can see the coefficient in front of the $\mathrm{x}$-term is just the square of $v$. Also we will define, $\gamma=b / m$, this is called the damping coefficient. Then we can rewrite the equation of motion:

$$
\frac{d x^{2}}{d^{2} t}+\gamma \frac{d x}{d t}+v_{0}^{2} x=\frac{q E}{m}
$$

This is called the Abraham-Lorentz equations. To find the solutions we should explicitly include the harmonic nature of the electric field by writing:

$$
E=E_{0} \cos (v t)
$$

By doing this, it hints to us to test the function of:

$$
x=A \cos (v t-\phi)
$$


Where $\phi$ is the phase. Then substitute this function into the equation of motion:

$$
\begin{aligned}
-v^{2} A \cos (v t-\phi)-A v \sin (v t-\phi)+A v_{0}^{2} \cos (v t-\phi) & =\frac{A q}{m} \cos (v t-\phi) \\
A\left(v_{0}^{2}-v^{2}\right) \cos (v t-\phi)-A v \sin (v t-\phi) & =\frac{A q E_{0}}{m} \cos (v t-\phi)
\end{aligned}
$$

Then applying the trigonometric identities $\sin (v t-\phi)=\sin (v t) \cos (\phi)-\cos (v t) \sin (\phi)$ and $\cos (v t-\phi)=\cos (v t) \cos (\phi)+\sin (v t) \sin (\phi)$, which leads to:

$$
\begin{aligned}
\left(A\left(v_{0}^{2}-v^{2}\right) \cos (\phi)\right. & \left.+A \nu \gamma \sin (\phi)-\frac{q E_{0}}{m}\right) \cos (v t) \\
& +A\left(\left(v_{0}^{2}-v^{2}\right) \sin (\phi)-\nu \gamma \cos (\phi)\right) \sin (\nu t)=0
\end{aligned}
$$

This equality is always true, so the terms in front of $\sin (v t)$ and $\cos (v t)$ must both be zero. So, then:

$$
A\left(v_{0}^{2}-v^{2}\right) \cos (\phi)+A v \gamma \sin (\phi)-\frac{q E_{0}}{m}=0
$$

And

$$
A\left(\left(v_{0}^{2}-v^{2}\right) \sin (\phi)-v \cos (\phi)\right)=0
$$

We have two equations and two unknowns, we can find $A$ and $\phi$. We find that:

$$
A=\frac{q E_{0}}{m} * \frac{1}{\left(\left(v_{0}^{2}-v^{2}\right)^{2}+v^{22}\right)^{\frac{1}{2}}}
$$

And

$$
\phi=\arctan \left(\frac{v \phi}{v_{0}^{2}-v^{2}}\right)
$$

These equations are useful in this form, particularly the phase but currently not for our present requirements. It is a lot of work but by using trigonometric identities, it is possible to show: 


$$
x=\frac{q E_{0}}{m} * \frac{1}{v_{0}^{2}-v^{2}-i v \gamma}
$$

Now lets us turn our attention to electrodynamics again. The dipole moment $\hat{p}$ of a single electron is just the charge of the electron times the displacement of the electron. The dipole moment is also the polarizability $\hat{\alpha}$ times the electric field, then we can write:

$$
\hat{\alpha}=\frac{q^{2}}{m} \frac{1}{v_{0}^{2}-v^{2}-i v \gamma}
$$

We can use the electrodynamic relations from the beginning of this section. For a single oscillator, the polarization is the polarizability and then we can write:

$$
\begin{aligned}
D & =\epsilon_{0} E+P \\
& =\epsilon_{0} E+\hat{p} \\
& =\epsilon_{0} E+\hat{\alpha}
\end{aligned}
$$

Since $D=\epsilon E$, this becomes:

$$
\begin{aligned}
\epsilon & =\epsilon_{0}+\hat{\alpha} \\
& =\frac{q^{2}}{m} \frac{1}{v_{0}^{2}-v^{2}-i v \gamma}
\end{aligned}
$$

This equation is important because $\epsilon$ determines the optical properties of the material and the unknown values of $\gamma$ and $v_{0}$ can be found through an examination of resonances in the reflection spectrum of the material. The real spectrum will have multiple resonances, and we can include this by adding additional oscillators with 
different $\gamma$ and $v_{0}$. We can show that the value of $\epsilon$ for such a collection of oscillators is the summations of individual $\epsilon$ terms (Bohren \& Huffman, 1983). In practice, we can fit a curve generated by Eq. (3.23) to the resonance in the spectrum, and then obtain an accurate value for $\epsilon$.

The dielectric function is useful but in general we are more used to thinking about the complex refractive index $m$, we will examine the relationship between these two parameters. By definition:

$$
m=\frac{c}{v_{p}}
$$

Where $c$ is the speed of light in vacuum and $v_{p}$ is the phase velocity of light in the medium. If we go back to Maxwells equations, one can show that the phase velocity is given by:

$$
v_{p}=\frac{c}{\sqrt{\epsilon \mu}}
$$

Where $\mu$ is the magnetic permeability of the material. Close to all solid materials have a $\mu$ very close to one, so then we can write:

$$
v_{p}=\frac{c}{\sqrt{\epsilon}}
$$

But from definition of the complex refractive index, we can write:

$$
\frac{c}{m}=\frac{c}{\sqrt{\epsilon}}
$$




$$
m=\sqrt{\epsilon}
$$

Then we can see that the relationship between complex refractive index and the dielectric function is straightforward.

\subsection{Light Propagation Through Dust}

As discussed earlier, dust shells form around AGB stars, and it is this dust in which we are interested. In order to research said dust, we must discuss the fundamentals of light propagation through a medium.

Let us consider, light with flux $F_{\lambda}$ propagating through a slab of dust of density $\rho$ and thickness $d x$ and the resulting flux emerging from the other side denoted as $F_{\lambda}^{\prime}$. This can be seen below in the Figure 3.1.

We can write the change in the flux, $d F_{\lambda}$ as the beam of light passes through the slab as:

$$
d F_{\lambda}=F_{\lambda}^{\prime}-F_{\lambda}
$$

There are three processes that cause the change in the flux: absorption, scattering and emission. There can be other processes such as fluorescence but these processes usually require high energy photons. Since out study concentrates on AGB stars which have a surface temperature of $3000 \mathrm{~K}$, there are no high energy photons available. We will not take fluorescence similar high energy processes into consideration here. 


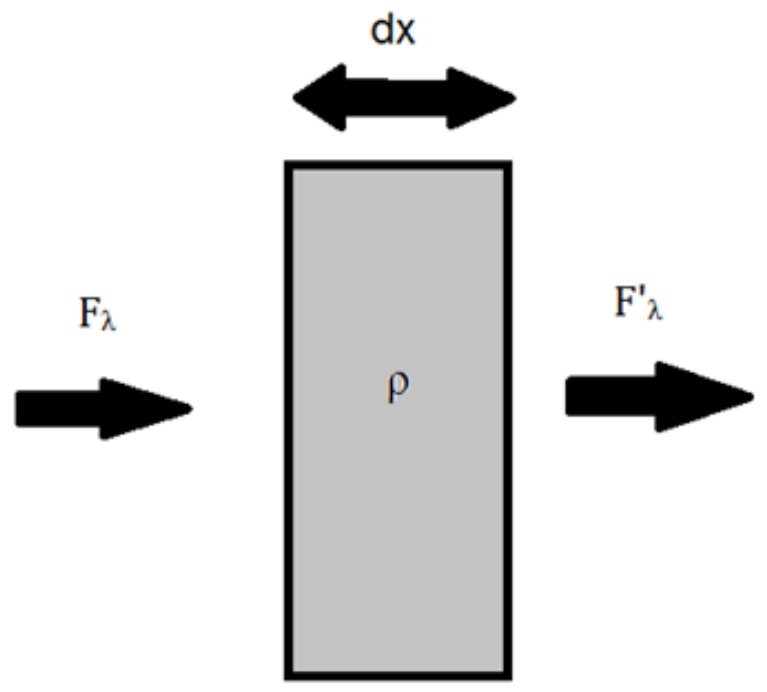

Figure 3.1: Light propagating through a slab of dust. Reference discussion for an explanation of terms.

\subsubsection{Extinction}

Ignoring emission of light from slab/dust, we would expect the flux emerging from the right side of the slab $F_{\lambda}^{\prime}$ to be less than the incident flux, for two reasons: absorption and reflection. Some of the incident light will be absorbed by the slab/dust and, light will be scattered be reflection from the grain/slab surface. Combined these processes are known as extinction. Lets define the wavelength-dependent opacity, $\chi_{\lambda}$ such that:

$$
d F_{\lambda}=-\chi_{\lambda} \rho F_{\lambda} d x
$$

By integration, we find that:

$$
F_{\lambda}(x)=C e^{-\chi_{\lambda} \rho x}
$$


Where $C$ is a constant of integration. We then can determine the value for the constant by observing that $x=0, F_{\lambda}(0)=C$, which leads us to:

$$
F_{\lambda}(x)=F_{\lambda}(0) e^{-\chi_{\lambda} \rho x}
$$

With $F_{\lambda}(0)$ as the flux before it interacts with the slab. From this, we can see the flux will exponentially decrease in intensity as it passes through the dust. This is called Beers Law. Lets now define the wavelength-dependent optical depth ${ }^{1} \tau_{\lambda}$ as:

$$
d \tau_{\lambda}=\chi(\lambda) \rho d x
$$

We can use substitution of this definition into the equation to get:

$$
F_{\lambda}(x)=F_{\lambda}(0) e^{-\tau_{\lambda}}
$$

Optical depth is unit-less and is a measure of the extinction coefficient or absorbance. It describes how much absorption occurs when light travels through an absorbing medium, such as an interstellar cloud. We can see this more explicitly by integrating the equation of $d \tau_{\lambda}$.

$$
\tau_{\lambda}=\int_{0}^{l} \chi(\lambda, x) \rho(x) d x
$$

Where $l$ is the length of the line of sight. We are looking through dust with $\tau<1$ we say the dust is optically thin, it if is greater than 1 we say it is optically thick.

\footnotetext{
${ }^{1}$ The optical depth is denoted in a more specific way by explicitly giving the wavelength at which the optical depth; for example, many optical depths in this research are at $10 \mu \mathrm{m}$ and are wrote as $\tau_{10 \mu m}$.
} 
If we go back to $F_{\lambda}(x)=F_{\lambda}(0) e^{-\tau_{\lambda}}$ we can obtain this result from another way that has useful consequences. Lets imagine radiation in the form of an ideal sinusoidal wave passing through the dust. This takes the form of:

$$
A=A_{0} e^{-i\left(\omega t-\frac{2 \pi x}{\lambda}\right)}
$$

Where $A_{0}$ is the amplitude, $\omega$ is the angular frequency, and $\lambda$ is the wavelength. The energy contained in this wave is proportional to $A^{2}$. As the wave enters the dust, it will slow downs by an amount governed by the complex index of refraction, $m=n+i k$. We can express this in the wave equation as:

$$
\begin{aligned}
A & =A_{0} e^{-i\left(\omega t-\frac{2 m \pi x}{\lambda}\right)} \\
& =A_{0} e^{-i\left(\omega t-\frac{2 \pi[n+i k]}{\lambda}\right)} \\
& =A_{0} e^{-i\left(\omega t-\frac{2 \pi n}{\lambda}\right)} e^{\frac{2 \pi k}{\lambda}}
\end{aligned}
$$

The first exponential is just a wave traveling with a velocity dependent on the real part of the refractive index, $n$, the second term is the exponential falloff. This is the same exponential falloff found in the equation (3.32), expect now we are only dealing with the opacity due to absorption. So the optical depth is equal to: $\tau=\rho \chi x=\kappa x$. Where $\kappa$ is the wavelength dependent absorption coefficient, $\kappa=\rho \chi$. Since the exponential term in equation (3.32) is the same as the term here (expect now that we have switched the absorption coefficient we no longer need to include the density), as this is already included in the term, we can write: 


$$
\begin{gathered}
-\kappa_{a} b s x=-\frac{2 m k x}{\lambda} \\
-\kappa_{a} b s=-\frac{2 m k}{\lambda}
\end{gathered}
$$

Lets consider what happens at the interfaces of the slab. Some of the light will be reflect, absorbed and some will pass through the dust. We are concerned with two reflections. One reflections when the light passes into the slab and another one occurs when the light meets the boundary on the right. At both of the interfaces, the light will reflect back to fraction $R$ of the total energy, and therefore the transmitted light $T$ at each interface is $(1-R)$. The light will continue to be absorbed as it continues through the dust. The equation for the amount of transmitted light, assuming the same fraction of light is reflected at each interfaces) is given by:

$$
T=(1-R)^{2} e^{-\tau_{\lambda}}
$$

This equation tells us that we can obtain $\kappa_{a b s}$ from measuring the amount of transmitted light, and equation (3.39) the value of $k$ too.

\section{Radiative transfer}

This section considers the change in the flux due to the opacity of the dust; there is also a change in the flux due to the thermal emission of the dust itself. Let's define the emission coefficient $j_{\lambda}$ such that the increase in flux $d F_{\lambda}$ as it passes through the slab is given by:

$$
d F_{\lambda}^{e m i s s i o n}=j_{\lambda} \rho d x
$$




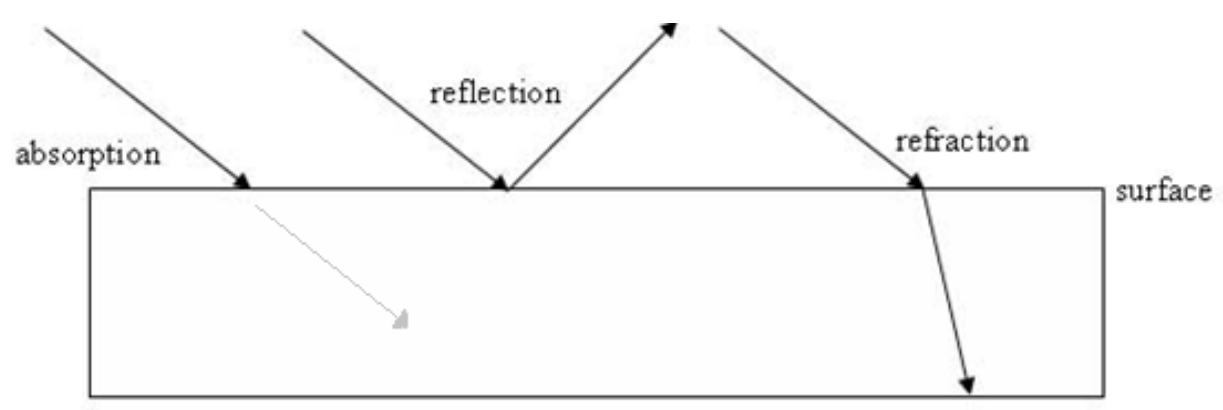

Figure 3.2: Diagram of absorption, refection and refraction of light

This leads us to write the total change in flux as:

$$
d F_{\lambda}=j_{\lambda} \rho d x-\chi_{\lambda} \rho F_{\lambda} d x
$$

If we divide this expression by $-\chi_{\lambda} \rho d x$ to obtain:

$$
-\frac{1}{\chi_{\lambda} \rho} \cdot \frac{d F_{\lambda}}{d x}=F_{\lambda}-\frac{j_{\lambda}}{\chi_{\lambda}}
$$

The fraction of $j_{\lambda} / \chi_{\lambda}$ is known as the source function, $S_{\lambda}$. Lets examine the term on the left side of the equation (3.43). By using the definition of optical depth, we can rewrite this as:

$$
-\frac{1}{\chi_{\lambda} \rho} \cdot \frac{d F_{\lambda}}{d x}=-\frac{d F_{\lambda}}{d \tau_{\lambda}}
$$

Then rewriting equation (3.43) provides us:

$$
\frac{d F_{\lambda}}{d \tau_{\lambda}}=S_{\lambda}-F_{\lambda}
$$


This equation is called the transfer equation and it forms the basis for radiatvie transfer. We can also integrate to obtain the transfer equation in another form:

$$
F_{\lambda}\left(\tau_{\lambda}\right)=F_{\lambda}(0) e^{-\tau_{\lambda}}+\int_{0}^{\tau_{\lambda}} S_{\lambda}\left(z_{\lambda}\right) e^{-\tau_{\lambda}-z_{\lambda}} d z_{\lambda}
$$

The transfer equation is generally very challenging to solve. Chandrasekhar (1950) put a great deal of effort into finding solutions and techniques to use the transfer equations. For some situations and geometries exact solutions do exist, however there needs to be some kind of numerical method used.

\subsubsection{Spectral Analysis}

We will investigate the methods we will use to analyze the spectra produced by dust in space, in particular the circumstellar shell around the carbon rich star. The emission at wavelength $\lambda$ from a dusty region with optical depth $\tau(\lambda)$ and a single temperature $T:$

$$
F_{\lambda} \propto B(\lambda, T)\left[1-e^{-\tau_{\lambda}}\right]
$$

where $B(\lambda, T)$ is the Planck function.

When the source is optically thick, $F_{\lambda} \propto B(\lambda, T) \times Q(\lambda)$, where $Q(\lambda)$ is the absorption/extinction/emission efficiency. There can be two interpretations of how to extract the absorption/emission properties of the circumstellar dust from a given an extinction efficiency factor for the entire spectrum: 


$$
F_{\lambda}=B B_{\lambda} \times Q_{\lambda}
$$

Or as though the entire spectrum is made up the sum of products of individual components.

$$
F_{\lambda}=\left[B B_{\text {carbon }} \times Q_{\text {carbon }}\right]+\left[B B_{S i C} \times Q_{S i C}\right]+\left[B B_{\text {other }} \times Q_{\text {other }}\right]
$$

Where $B B$ is the temperature-dependent Planck function and $Q \propto$ absorbance. Absorbance is essentially optical depth.

Using the continuum-divided spectrum assumes the type of spectrum described above. Dividing the entire spectrum by the fitted underlying continuum, leaves only the absorption/extinction efficiency of the dust, assuming all dust species have the same blackbody temperature. In our case, we effectively end up with an optical depth spectrum which is equivalent to extinction efficiency for some sort of composite spectrum which is equivalent to an extinction efficiency for some sort of composite grains that represents all the grains present.

In reality the situation is more complex:

$$
F_{\lambda}=\sum_{i=1, j=1}^{n, m} B B_{i} \times Q_{j}
$$

Where $B B_{i}$ represents a single dust or stellar temperature and each $Q_{j}$ represents the extinction efficiency for a single grain type as defined by its size, shape, composition and crystal structure. Complex situations can only be completely addressed using radiative transfer modeling. 


\section{Chapter 4}

\section{Observations of Carbon Stars and}

\section{the Dust Condensation Sequence}

"Second star to the right and straight on 'til morning." - Peter Pan (Peter Pan)

\subsection{Introduction}

In $\S 1.2 .5$ we talked about the dust shell that forms around the AGB stars with a $\mathrm{C} / \mathrm{O}>1$. We analyzed the spectra of a collection of nine $\mathrm{C}$ stars, modeling 1-shell and 2-shell models. We did this with the help of the molecular features in the spectra of the carbon rich star. 


\subsection{1 $\sim 11 \mu \mathrm{m}$ Feature}

The $\sim 11 \mu \mathrm{m} \mathrm{SiC}$ feature and its characteristics that are present in carbon stars have been discussed by Baron et al. 1987; Willems, 1988; Chan \& Kwik, 1990; Goebel et al., 1995; Speck et al. 1997; Sloan et al., 1988; Speck et al., 2005. Datasets such as the Infrared Astronomical Satellite (IRAS; Neugebauer et al., 1984) Low Resolution Spectrometer (LRS), the Infrared Space Observatory (ISO; Kessler et al., 1996), Short Wavelength Spectrometer (SWS; de Graauw et al., 1996) and also the United Kingdom Infrared Telescope (UKIRT) have been used to examine the $\sim 11$ $\mu \mathrm{m} \mathrm{SiC} \mathrm{feature.} \mathrm{All} \mathrm{these} \mathrm{analyses} \mathrm{looked} \mathrm{at} \mathrm{the} \mathrm{strength} \mathrm{and} \mathrm{peak} \mathrm{positions} \mathrm{of} \mathrm{the}$ $\sim 11 \mu \mathrm{m} \mathrm{SiC} \mathrm{feature.}$

One reason that there are many studies about the $\sim 11 \mu \mathrm{m} \mathrm{SiC}$ feature is because of the polytypes of $\mathrm{SiC}$ which can form around the carbon star and the exact spectral feature that these polytypes would produce; the crystal structure of $\mathrm{SiC}$ grains contains important information about the environment these grains formed within and also their cooling history.

\subsection{Observation}

We examined the spectra of nine known carbon stars observed spectroscopically using IRAS LRS, ISO SWS and the BVJK light band (See table 5.1).

The raw ISO data was extracted from the ISO data archive, the IRAS data set and BV JK light band data set were obtained from SIMBAD Astronomical Database (http://simbad.u-strasbg.fr/simbad/). 
Table 4.1: Target Sources

\begin{tabular}{llrr} 
Star name & Other names & TDT number & Observation date \\
\hline VX And & $00172+4425$, IRC+40006 & 42801502 & 1997Jan17 \\
R Scl & $01246-3248$, IRC-30015 & 38603503 & 1996Dec19 \\
W Ori & $05028+0106$, IRC+00066 & 85801604 & 1998Mar22 \\
SS Vir & $12226+0102$. IRC+00217 & 21100138 & 1996Jun14 \\
RY Dra & $12544+6615$, IRC+70116 & 54300203 & 1997May12 \\
S Sct & $18476-0758$, IRC-10467 & 16401849 & 1996Apr29 \\
V Aql & $19017-0545$, IRC-10486 & 16402151 & 1996Sept21 \\
V460 Cyg & $21399+3516$, IRC+40489 & 42201734 & 1997Jan11 \\
TX Psc & $23438+0312$, IRC+00532 & 75700419 & 1997Dec11 \\
\hline
\end{tabular}

\subsection{Radiative Transfer Modeling}

Radiative transfer (RT) modeling has been used extensively in investigating carbonrich stars (Volk et al. 1992; Groenewegen 1995; 2000). We used the one-dimensional radiative transfer code DUSTY (Ivezic \& Elitzur 1995; Nenkova et al. 2000), to determine the effect of dust shell parameters on the merging spectra from carbon rich stars. There are many input parameters needed for RT modeling, which need some introduction. For simplicity, dust grains are assumed to be spherical. DUSTY is able to include other grain shapes, but this expands the parameter space to create more degeneracy between models and is far beyond the scope of the present work. 


\subsubsection{Radial Dust Density Distribution}

We assume a radial dust density distribution of $\frac{1}{r^{2}}$ which reflects a constant mass-loss rate. This choice can be controversial and therefore will be justified. Current dust formation models suggest that carbon star mass loss is expected to be modulated on several different timescales, especially that of the pulsation cycle (Woitke 2006, and reference therein). Woitke (2006) also suggested that the dynamics in the dustforming zones around carbon stars lead to inhomogeneous dust formation, producing fine scale structure in the density of the dust envelope. Pulsation shocks are expected to have a strong effect on local conditions (e.g. Cherchneff 2006), this is not reflected in temporal changes in the IR spectra of carbon stars. Rowan-Robinson \& Harris (1983) showed that carbon star spectra can be well fitted using a density distribution of $\frac{1}{r^{2}}$.

In our models, we assumed that modulations in density have been wiped out or insignificant in determining the spectrum. We are clearly going to be seeing spectra that are dominated by the inner most, densest layer of dust, which is hottest and closest to the star. The outer dust is low enough density and cool enough that its contribution (at least in the mid IR, where spectral features occur) are minimal.

\subsubsection{Modeling Grain-Size Distributions}

Our initial modeling studies used a grain size distribution of MRN (i.e. n(a) proportional to $a^{-q}$, where $\mathrm{n}$ is the number of the grains in the size interval $(a, a+d a)$ and $q=3.5, a_{\min }=0.005 \mu \mathrm{m}$ and $a_{\max }=0.25 \mu \mathrm{m}$; Mathis et. al 1977) This was 
chosen because we do not actually know the grain-size distribution and MRN is as plausible as any other. The MRN distribution was developed for interstellar dust where the balance of formation and destruction is different from AGB circumstellar environments. The grain-size distribution created in circumstellar environments of AGB stars has been suggested to have a steeper power law (i.e. $a^{5}$ ) by Dominik et al. (1989) but Kim et al. (1994, KMH) modified the MRN distribution to include an exponential fall-off term. Groenewegen (1995) argued that specific grain size is not important as long as the grains are small enough that the absorption and scattering properties are independent of the grain size. Given the arguments for the population of large grains, it is unrealistic limiting the grain size to smaller than $0.1 \mu m$. We generated models using MRN.

\subsubsection{Parameter Space Investigated}

The variables investigated with DUSTY are the inner dust shell temperature $\left(T_{i n}\right)$, radial density distribution, optical depth (specified at $0.55 \mu m ; \tau_{0.55 \mu m}$ ), dust composition, and geometric thickness of the dust shell, $\xi=R_{\text {out }} / R_{\text {in }}$, where $R_{\text {out }}$ and $R_{\text {in }}$ are the outer and inner radii of the dust shell. The optical constants for the dust components came from Pegourie (1988), Hanner (1988) and Draine \& Lee (1984) for $\mathrm{SiC}$, graphite, amorphous carbon respectively. The iron optical constants came from Speck et al (2015). 


\section{Degeneracies in radiatve transfer modeling}

There is a clear degeneracy in the models because of the relationship between certain parameters, e.g. optical depth and geometric shell thickness, or optical depth and the temperature at the inner edge of the dust shell. The degeneracies and the relationship between the different input parameters in radiative transfer models, were discussed by Ivezic \& Elitxur (1997); We need to understand the relationship of the parameters to understand what our models mean.

The wavelength-dependent optical depth, $\tau_{\lambda}$ is defined by:

$$
d \tau_{\lambda}=\rho(r) \chi_{\lambda} d r
$$

where $\rho(r)$ is the density of the absorbing/scattering particles (i.e. dust grains) and $\chi_{\lambda}$ is the wavelength dependent opacity of the assemblage of particles along the line of sight.

$$
\tau_{\lambda}=\int_{R_{\text {in }}}^{R_{\text {out }}} \rho(r) \chi_{\lambda} d r
$$

where $R_{\text {in }}$ is the inner dust shell radius and $R_{\text {out }}$ is the outer dust shell radius. While $\chi_{\lambda}$ is the dependent on the density distribution of the grains, maintaining the size, shape and composition (and crystal structure) of the grains means that $\chi_{\lambda}$ will not change significantly. We assume that $\chi_{\lambda}$ is constant, for simplicity. In our models

we assume the density of the dust shell drops off as $1 / r^{2}$ from the central star. In addition, our models specify the relative geometrical thickness of the dust shell as $\xi=R_{\text {out }} / R_{\text {in }}$. Then we get: 


$$
\tau_{\lambda}=\chi_{\lambda}\left[\frac{\xi-1}{\xi R_{\text {in }}}\right]
$$

The value of $R_{\text {in }}$ is set by the values we input for the star's effective temperature and the inner dust radius or the condensation temperature $\left(T_{i n}\right)$. If the temperature and luminosity of the star, we can use $T(r) \propto r^{\frac{-1}{2}}$ temperature distribution to determine the relationship between $T_{i n}$ and $R_{\text {in }}$ and substituting into equation 4.3 , it becomes:

$$
\tau_{\lambda}=\sqrt{\frac{16 \pi \rho}{L_{*}}} \chi_{\lambda} T_{i n}^{2}\left[\frac{\xi-1}{\xi}\right]
$$

Here we assume that the grains are blackbodies since they are mostly carbon. By including the albedo this would allow for a more accurate calculation, but then this would depend on the detailed dust parameters (i.e. crystal structure), and the error made by our assumption is small (i.e. significantly less than an order of magnitude).

Equation (4.4), shows us that for a dust shell with a constant relative shell thickness $(\xi)$ the optical depth should increase with the square of the inner dust temperature. Similarly, if the inner dust temperature is fixed, then increasing the relative shell thickness should decrease optical depth a bit (as $\left.\frac{\xi-1}{\xi}\right)$; this effect will become negligible for large geometric sizes. 


\subsection{Determining the Dust Condensation Temper- ature}

\subsubsection{Theoretical Dust Condensation Models}

In earlier studies, the value of the dust condensation temperatures can bring degeneracy on radiative transfer modeling. To help reduce, the degeneracy we turn the dust condensation theory to find a theoretical dust condensation temperature, (i.e. $T_{\text {in }}$ ) which is appropriate for our investigated stars.

Many presolar grains can be attributed to carbon stars and need to be investigated for dust formation regions. For instance, in presolar grains, titanium carbide (TiC) is found in the center of carbon $(\mathrm{C})$ grains from AGB stars; only one $\mathrm{SiC}$ grains have been found coated in carbon (Clayton \& Nittler, 2004; Bernatowicz et al., 2005). Therefore, there have been numerous studies done over the theoretical condensation sequence in Galactic carbon stars in attempt to constrain the physical parameters of the dust condensation regions. The studies showed that the condensation sequence of $\mathrm{C}, \mathrm{SiC}$ and $\mathrm{TiC}$ are dependent on various parameters, especially the $\mathrm{C} / \mathrm{O}$ ratio and gas pressure (Gas pressure is a measure of the mass-loss $(\dot{M})$ intertwined with the photospheric temperature $\left(T_{*}\right)$ and the outflow velocity $\left(v_{e x p}\right)$ ) (Lodders \& Fegley, 1995; Sharp \& Wasserburg, 1995).

Carbon forms at higher condensation temperature, closer to the star than $\mathrm{SiC}$, decreases, has been argued by Sharp \& Wasserburg (1995). There is a significant decrease in the amount of carbon available in the gas, and therefore $\mathrm{SiC}$ and carbon 
do not form simultaneously, resulting in naked $\mathrm{SiC}$ grains. So, for Glaactic sources, the condensation sequence in the majority of carbon stars should be: TiC-C-SiC, to be able to produce $\mathrm{TiC}$ grains the uncoated $\mathrm{SiC}$ grains seen in the meteoritic presolar grains samples. Speck et al. (2005) found observational evidence for uncoated SiC grains. From kinetic and stellar model considerations, dust grains should form in the pressure range $2 \times 10^{-7}<P<4 \times 10^{-4}$ (Sharp \& Wasserburg 1995).

Lodders \& Fegley (1995) modeled the effect of C/O and pressure on the condensation sequence in carbon stars, also the effect of s-process and nitrogen abundances and also discussed the effect of metallicity. The general trends in condensation temperatures were to found to be: 1) all condensation temperatures decrease as the gas pressure decrease, and 2) At $\mathrm{C} / \mathrm{O}>1$ the condensation temperature of graphite increases with $\mathrm{C} / \mathrm{O}$, for a given pressure and otherwise constant composition.

Figure 4.1 shows how the condensation temperature of carbon and $\mathrm{SiC}$ vary with the gas pressure in the dust condensation zone.

From what is seen in the Figure 4.1, carbon always forms first from a cooling gas. Very high pressures are required for $\mathrm{SiC}$ to form before carbon. For solar metallicity and $\mathrm{C} / \mathrm{O}=1.05, \mathrm{SiC}$ forms before carbon when $\mathrm{P} \geq 3.4 \times 10^{-5}$. As the $\mathrm{C} / \mathrm{O}$ increases, the minimum pressure required to form $\mathrm{SiC}$ first increases. When $\mathrm{C} / \mathrm{O}$ is $\sim 1.5$, carbon will form before $\mathrm{SiC}$. The exact $\mathrm{C} / \mathrm{O}$ ratio at which the carbon forms before $\mathrm{SiC}$ depends on pressure. So, in order to account for observations of $\mathrm{SiC}$ features in the Galaxy, and the presolar grain record, we can restrict the P-C/O space such that, for $\mathrm{C} / \mathrm{O}$ the gas pressure must remain low; for higher $\mathrm{C} / \mathrm{O}$ the pressure can be higher. This can be applied to constrain the dust forming environment around 


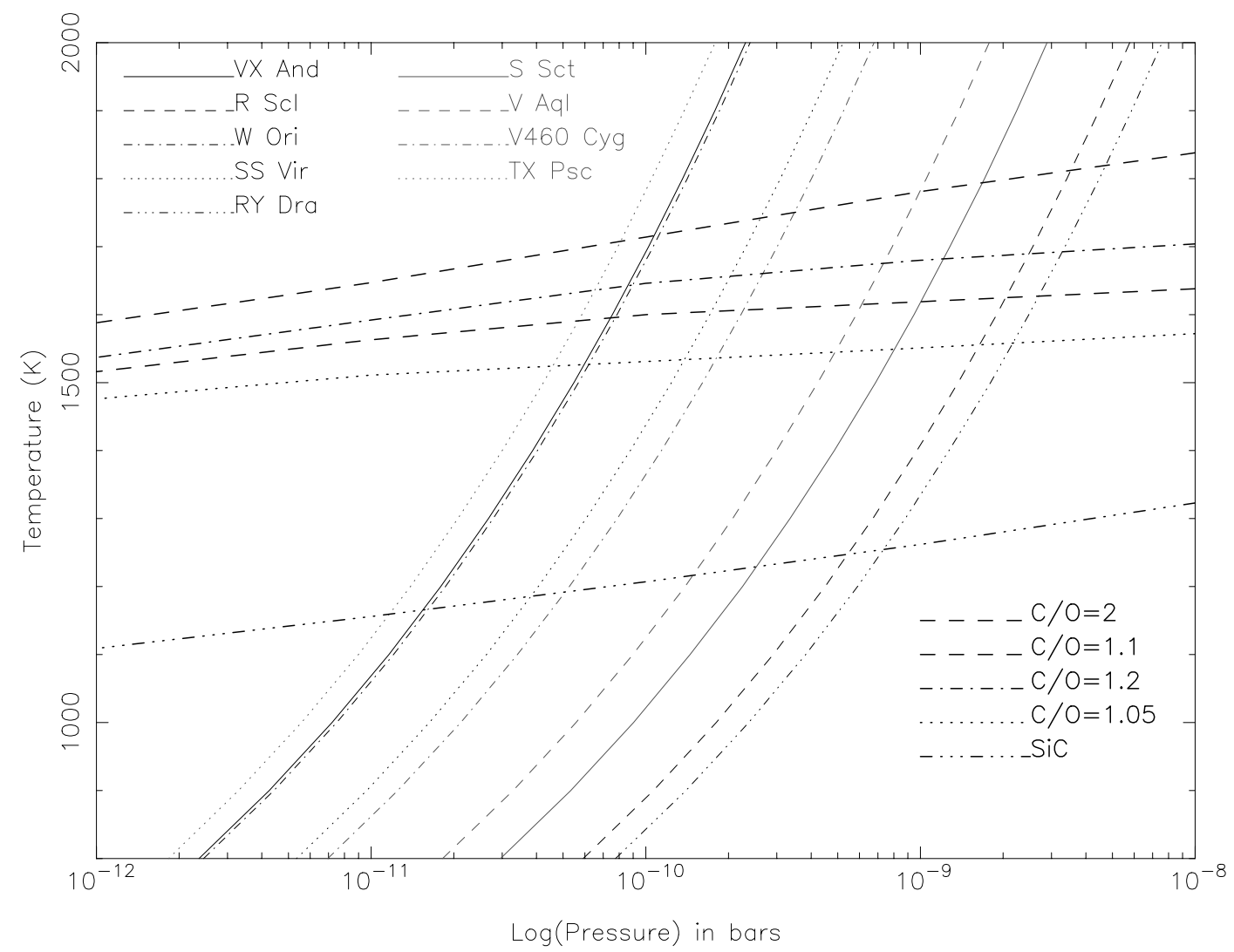

Figure 4.1: Pressure-temperature space for the dust condensation region around carbon stars. The gray lines indicate the condensation temperature for a given pressure as calculated by Lodders \& Fegley (1995). The black lines indicate the P-T paths for the out-flowing gas from our sample stars. 
Galactic carbon stars.

Carbon star spectra studies in the Galaxy and Magellanic Clouds have lead to different interpretations with respect to the condensation sequence. Lagadec et al. (2007) said for a sequence in which carbon forms after $\mathrm{SiC}$ in the Galaxy, unlike where $\mathrm{SiC}$ and carbon form together in the Large Magellanic Cloud (LMC) and the sequence is reversed ( $\mathrm{C}$ and then $\mathrm{SiC}$ ) in the Small Magellanic Cloud (SMC). Also they suggest, that the change in the strength of the features is due to the lower number of Si atoms available for $\mathrm{SiC}$ formations. The proposed Galactic condensation sequence is at odds with both the models and the meteoritic evidence. Condensation sequence in which carbon forms before $\mathrm{SiC}$ first for Galactic carbon stars, Leisenring et al. (2008) supported, also this is followed by the simultaneous condensation of $\mathrm{SiC}$ and carbon. Speck et al. (2006) used Lodders \& Fegley (1995) model to explain an unusual LMC carbon star spectrum, which suggests that the condensation sequence is sensitive to both mass-loss rate and metallicity.

\section{P-T space in the condensation zone around carbon AGB stars}

To constrain the input parameters in the models we need to be able to find out the pressure-temperature space in the dust condensation zone. For a star with a mass-loss rate $\dot{M}$ and an expansion velocity of $v_{\text {exp }}$, the density $\rho$ of the circumstellar shell at a radius $r$ it can be given as:

$$
\rho=\frac{\dot{M}}{4 \pi r^{2} v_{\text {exp }}}
$$

If the temperature and luminosity of the star is known and also the composition 
of the outflowing material, then we can combine this information with the Ideal Gas Law $(P V=n R T)$ and $T(r) \propto 1 / \sqrt{r}$ temperature distribution to determine the gas pressure at the condensation radius, where the condensation radius is the distance from the star where the gas has the condensation temperature $\left(T_{\text {cond }}\right)$.

The solid and gas phases are assumed to be at the same temperature, for simplicity purposes. Even though this is a clear simplification (e.g. Chigai \& Yamamoto, 2003), the temperature difference is small compared to the difference needed to significantly affect the dust formation. The outflowing material is assumed to be atomic hydrogen, mostly. It's probably a mixture between atomic and molecular hydrogen $\left(\mathrm{H}_{2}\right)$ because $\mathrm{H}_{2}$ forms around $2000 \mathrm{~K}$ and the temperture in the outflow is decreasing from the stellar surface temperature of $\sim 3000 \mathrm{~K}$ to the dust condensation temperature in the 1000-8000 K range. If entirely molecular hydrogen gas, it would cut in half the gas pressure compared to the atomic gas. Also, we assumed published CO outflow velocities, which reflect the speed of the outflowing material after radiation pressure acceleration. If we used the pre-dust-formation outflow speed of $\approx 5 \mathrm{~km} / \mathrm{s}$, this would increase the pressure. By using the published values for our sample stars' mass-loss rates, luminosities, and expansion velocities, we can estimate where the stars' dust condensation zones fall in the P-T space.

\section{Comparison of P-T for dust condensation models and sample stars}

From Figure 4.1 we can see the trend of the P-T space for the dust condensation zone for our sample stars. It is visible that the pressure is never high enough for $\mathrm{SiC}$ to form before carbon. This agrees with meteoritic evidence which suggests that $\mathrm{SiC}$ 
does not get coated in carbon and supports the idea that variations in strength of the $\mathrm{SiC}$ feature are related to self-absorption.

The condensation temperature and the pressure in the gas from which the dust forms is dependent on each other. Also, the P-T space suggests that that the carbon grains will form at temperatures $\approx 1600 \mathrm{~K}$. If the $\mathrm{C} / \mathrm{O}$ is extremely high, then the graphite can form as high as $\sim 1800 \mathrm{~K}$. The Galactic carbon stars in which the $\mathrm{C} / \mathrm{O}$ ratio has been measured, have a range between 1 to 1.8 , with a mean $\mathrm{C} / \mathrm{O}$ ratio of 〜1.15 (Lambert et al., 1986; Olofsson et al., 1993 a,b)

\subsubsection{Constraining Dust Composition}

Since we are studying carbon-rich stars, we limited the models to only including carbonaceous species: graphite, amorphous carbon, and silicon carbide. We did include iron into some of our models, this will be discussed later. The choice of carbon grains is equivocal. We do not know if the grains are composed mostly of carbon are glassy, poly-noncrystalline or well-ordered. From meteoritic presolar "graphite" grains, they suggest that circumstellar dust can contain either well-ordered graphite or polynanocrysyalline-graphene grains. Nevertheless, even the disordered graphene sheets are considered to be more graphitic than amorphous.

Amorphous carbon, pretty much consists of a mixutre of $s p^{2}$ (graphite-like) and $s p^{3}$ (diamond-like) carbon bonds. When heated, $s p^{3}$ bonds normally converts to $s p^{2}$ bonds, which graphitizing the amorphous carbon; it will remain dense like the amor-

phous phase $\left(2.8 \mathrm{~g} / \mathrm{cm}^{3}\right.$; Comelli et al., 1988; Saada, 2000; Kelires, 1993). When 
temperatures are $\sim 1300 \mathrm{~K}$ the fraction of graphite bonds is $\sim 90 \%$. In $\S 4.4 .1$, the comparison of the gas pressure in the circumstellar outflow to graphite formation temperature, showed that graphite should be able to form at temperatures significantly above $1300 \mathrm{~K}$. In the solid state, carbon forms as a chaotic solid, and at these temperatures the carbon would quickly anneal to a graphitic form.

\section{Iron dust grains}

An element that is often overlooked in modeling carbon stars is iron. Even in Orich environments, theoretical models suggest that iron should condense into metallic iron grains rather than oxides (Lodders \& Fegley, 1998). In Carbon-rich (reducing) environments, iron can condense as metallic iron, iron/nickel alloy, or iron carbide in carbon-rich environments between 2000 and $900 \mathrm{~K}$ with the nature of the major iron carrying phase being dependent on the total pressure (Salpeter 1977). Once iron leaves the stellar wind it must condense into solid form: while it is difficult to find chemical abundances in the gas phase of AGB winds, iron is strongly depleted from circumstellar environments of other evolved objects. Metallic iron is difficult to recognize since, like carbon grains, it only emits a continuum emission without any diagnostic spectral features. It is almost impossible to differentiate between carbon and iron grains spectroscopically (McDonald 2011). No studies on the estimate of abundances of iron grains have been carried out in carbon-rich AGB circumstellar envelopes (Willacy 2003).

In order to consider whether the iron will be a significant constituent of the dust

shells of our sample of carbon stars, we used the $\mathrm{C} / \mathrm{O}$ ratio and the abundance of 
iron as listed in Table 5.3 to estimate the relative abundance of iron versus carbon available for dust formation. In order to assess the effect of replacing carbon with iron in order RT models we made a codel for each star in which the continuum is assumed to be dominated by iron, with zero contribution from either amorphous carbon or graphite. In this way we can determine whether our results are sensitive to the precise composition of the dust shell. 


\section{Chapter 5}

\section{Transfer Modeling Selection and}

\section{Results}

"Kowalski, we'll be rich. The laws of physics don't apply to us." (After being told that a solid gold plane would not fly) - Skipper (Madagascar 3)

\subsection{Target Selection and Observations}

Our sample was selected according to the availability of the relevant data in the published literature. Carbon stars were selected if they met two criteria: (1) high resolution ISO SWS spectroscopic data (SIMBAD Astronomical Database) were also available and can be found in Table 5.1; and (2) detailed measurements of surface chemistry from visible spectroscopy were available (Abia 2015; J. Bergeat 2004; Guan- 
dalini et al. 2006; Lambert 1986; Danilovich 2015; Olofsson et al 1993; Olofsson 1992, Ivezic 1995; Schoier \& Olofsson 2001; Loup 1993) and can be found in Tables 5.2 and 5.3. Criterion 2 necessitates that our sample stars have low mass-loss rates such that their dust shells are sufficiently optically-thin to allow abundance analysis of the stars photospheres.

In addition to these two criteria, we also collated data on our sample that would help constrain our radiative transfer modeling. We have collated spectral data including the BV and JHK photometric bands, the IRAS 12, 25, 60 and $100 \mu$ m photometric measurements and the ISO SWS spectra. Using American Association of Variable Star Observers (AAVSO) database we also acquired the V-band magnitude on the day of observation, which allowed use to fit our models well over a large wavelength range.

To constrain the model input parameters, we used published mass-loss rates, expansion velocities, $\mathrm{C} / \mathrm{O}$ and theoretical dust condensation models to estimate the dust condensation temperature. The published data and their sources are listed in Tables 5.2 and 5.3 and can be seen in Figure 5.1.

\subsubsection{CrB}

Originally, V CrB was in the sample of carbon-rich stars. There are great complexities with modeling the spectrum. Reasons are: $\mathrm{V} \mathrm{CrB}$ is a cool carbon rich star, the temperature is quite poorly known, and no good effective temperature scale is avail- 


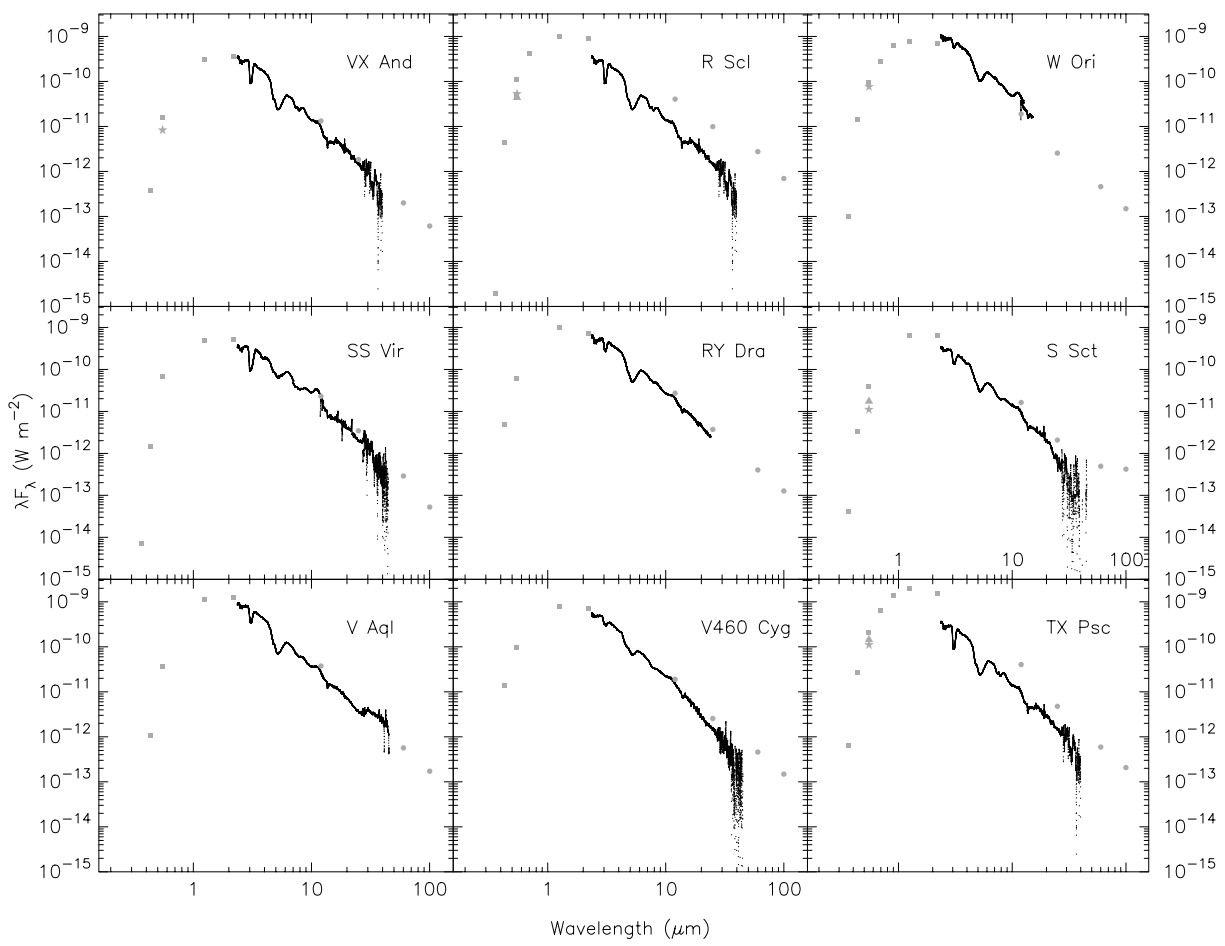

Figure 5.1: The photometrics bands, phtoemertics measurements and the ISO SWS spectra of our target sample. The BV and JHK photometric bands (displayed as squares), the IRAS 12, 25, 60 and $100 \mu \mathrm{m}$ photometric measurements (displayed as circles) and the ISO SWS spectra (displayed as a dark dotted line). 
Table 5.1: Sample of optically thin carbon stars

\begin{tabular}{llrr} 
Star name & other names & TDT number & Observation date \\
\hline VX And & $00172+4425$, IRC+40006 & 42801502 & 1997Jan17 \\
R Scl & $01246-3248$, IRC-30015 & 38603503 & 1996Dec19 \\
W Ori & $05028+0106$, IRC+00066 & 85801604 & 1998Mar22 \\
SS Vir & $12226+0102$. IRC+00217 & 21100138 & 1996Jun14 \\
RY Dra & $12544+6615$, IRC+70116 & 54300203 & 1997May12 \\
S Sct & $18476-0758$, IRC-10467 & 16401849 & 1996Apr29 \\
V Aql & $19017-0545$, IRC-10486 & 16402151 & 1996Sept21 \\
V460 Cyg & $21399+3516$, IRC+40489 & 42201734 & 1997Jan11 \\
TX Psc & $23438+0312$, IRC+00532 & 75700419 & 1997Dec11 \\
\hline
\end{tabular}

able for C-classifications. There has been a wide range of temperatures suggested for V CrB ranging from 1975-2300K. The range of C/O ratio was between 1-1.23 (Kipper 1998). With these variations in temperatures and $\mathrm{C} / \mathrm{O}$ ratio, $\mathrm{V}$ CrB was not used for the sample.

\section{$5.2 \quad$ Modeling Results}

The results of the radiative transfer modeling can be seen in Figures 5.2-5.8. The parameters used in each case can be seen in Tables 5.4-5.10. For each source, we have produced 1-shell models using dust compositions of graphite/SiC, AmC/SiC, iron/SiC, graphite/iron/SiC, $\mathrm{AmC} / \mathrm{iron} / \mathrm{SiC}$ and 2-shell models using graphite/ $\mathrm{SiC}$ and $\mathrm{AmC} /$ Sic. 
Table 5.2: Collected data for our carbon star sample

\begin{tabular}{lcccc}
\hline Name & $\mathrm{L}\left[\mathrm{L}_{\odot}\right]$ & $v_{\text {exp }}\left[\mathrm{km} \mathrm{s}^{-1}\right]$ & $\dot{M}\left[M_{\odot} \mathrm{yr}^{-1}\right]$ & Ref. $^{a}$ \\
\hline VX And & 3940 & 14 & $1.30 \times 10^{-7}$ & O93 \\
R Scl & 7080 & 18.2 & $7.60 \times 10^{-6}$ & L93 \\
W Ori & 2600 & 11 & $7.0 \times 10^{-8}$ & S00 \\
SS Vir & 5400 & 12.5 & $3.6 \times 10^{-7}$ & B04 \\
RY Dra & 840 & 10 & $6.5 \times 10^{-7}$ & L93 \\
S Sct & 4900 & 17.3 & $2.0 \times 10^{-8}$ & O92 \\
V Aql & 6500 & 11 & $1.30 \times 10^{-6}$ & D15 \\
V460 Cyg & 2600 & 10 & $1.80 \times 10^{-7}$ & S01 \\
TX Psc & 9300 & 11.8 & $2.0 \times 10^{-7}$ & I95 \\
\hline
\end{tabular}

Notes: ${ }^{a}$ References for the mass-loss rates, for luminosity and for the outflow velocities, D15-Danilovich (2015), L93-Loup (1993), O93-Olofsson et al (1993), O92-Olofsson (1992), I95-Ivezic 1995, B04-Bergeat et al (2004), and S01-Schoier and Olofsson (2001). 
Table 5.3: Collected data for our carbon star sample

\begin{tabular}{lcccccc} 
Name & $\mathrm{T}_{\star e f f}^{a}[\mathrm{~K}]$ & $\mathrm{D}^{b}[\mathrm{pc}]$ & $g / d^{c}$ & $\mathrm{Fe} / \mathrm{H}^{d}$ & $\mathrm{C} / \mathrm{H}^{e}$ & $\mathrm{C} / \mathrm{O}^{f}$ \\
\hline VX And & 3200 & 640 & 4.7 & -0.1 & -0.18 & 1.76 \\
R Scl & 2830 & 380 & 0.86 & $-0.5 *$ & -0.59 & 1.34 \\
W Ori & 2680 & 220 & 2.5 & 0.0 & -0.35 & 1.16 \\
SS Vir & 2560 & 560 & 1.4 & - & - & 1.05 \\
RY Dra & 2500 & 550 & 0.27 & $-0.05 \star$ & -0.38 & 1.18 \\
S Sct & 2610 & 540 & - & -0.1 & -0.11 & 1.07 \\
V Aql & 2895 & 330 & 1.0 & -0.05 & -0.20 & 1.25 \\
V460 Cyg & 2845 & 260 & 0.78 & -0.5 & -0.32 & 1.062 \\
TX Psc & 3010 & 370 & 0.72 & -0.4 & -0.32 & 1.027 \\
\hline
\end{tabular}

${ }^{a}$ Reference for effective temperature $\left(\mathrm{T}_{\star e f f}\right)$, Bergeat (2000).

${ }^{b}$ References for distances Guandalini et al 2006.

${ }^{c} g / d=10^{3} r$ where $r=\frac{\dot{M}_{d}}{\dot{M}_{g}}$ is the dust-to-gas ratio of mass-loss rate (Bergeat et al 2004).

${ }^{d}$ Reference for iron $(\mathrm{Fe})$ to hydrogen $(\mathrm{H})$ ratio, Abia et al (2009). * is from Abia (2015). * is from Abia (2009)/

${ }^{e}$ Reference for carbon (C) to hydrogen (H) ratio, Lambert (1986).

${ }^{f}$ Reference for the C/O (Bergeat et al 2004). 


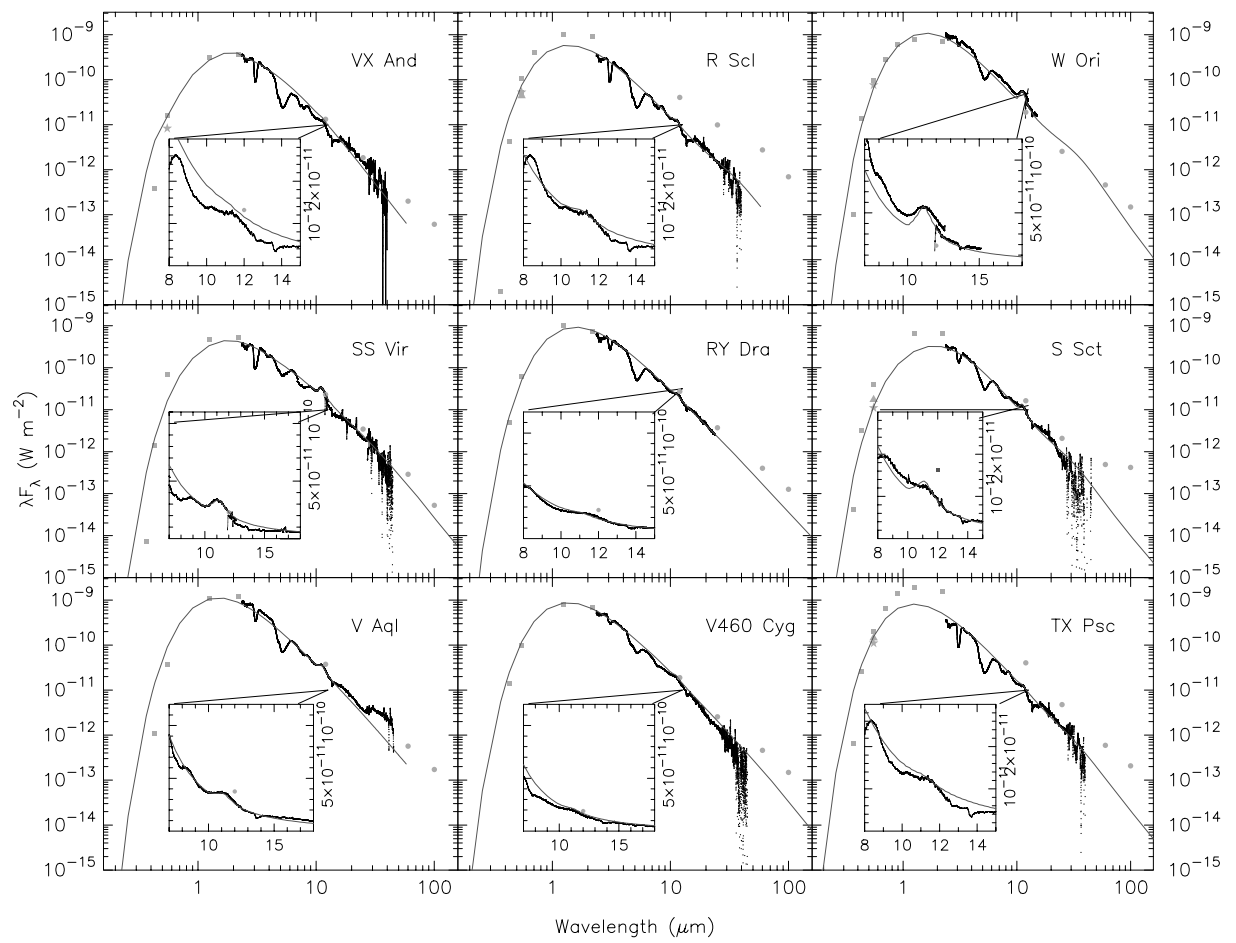

Figure 5.2: The photometrics bands, phtoemertics measurements and the ISO SWS spectra of our target sample. The BV and JHK photometric bands (displayed as squares), the IRAS 12, 25, 60 and $100 \mu \mathrm{m}$ photometric measurements (displayed as circles), the ISO SWS spectra (displayed as a dark dotted line), and and the modeled continuum (thick grey line). 


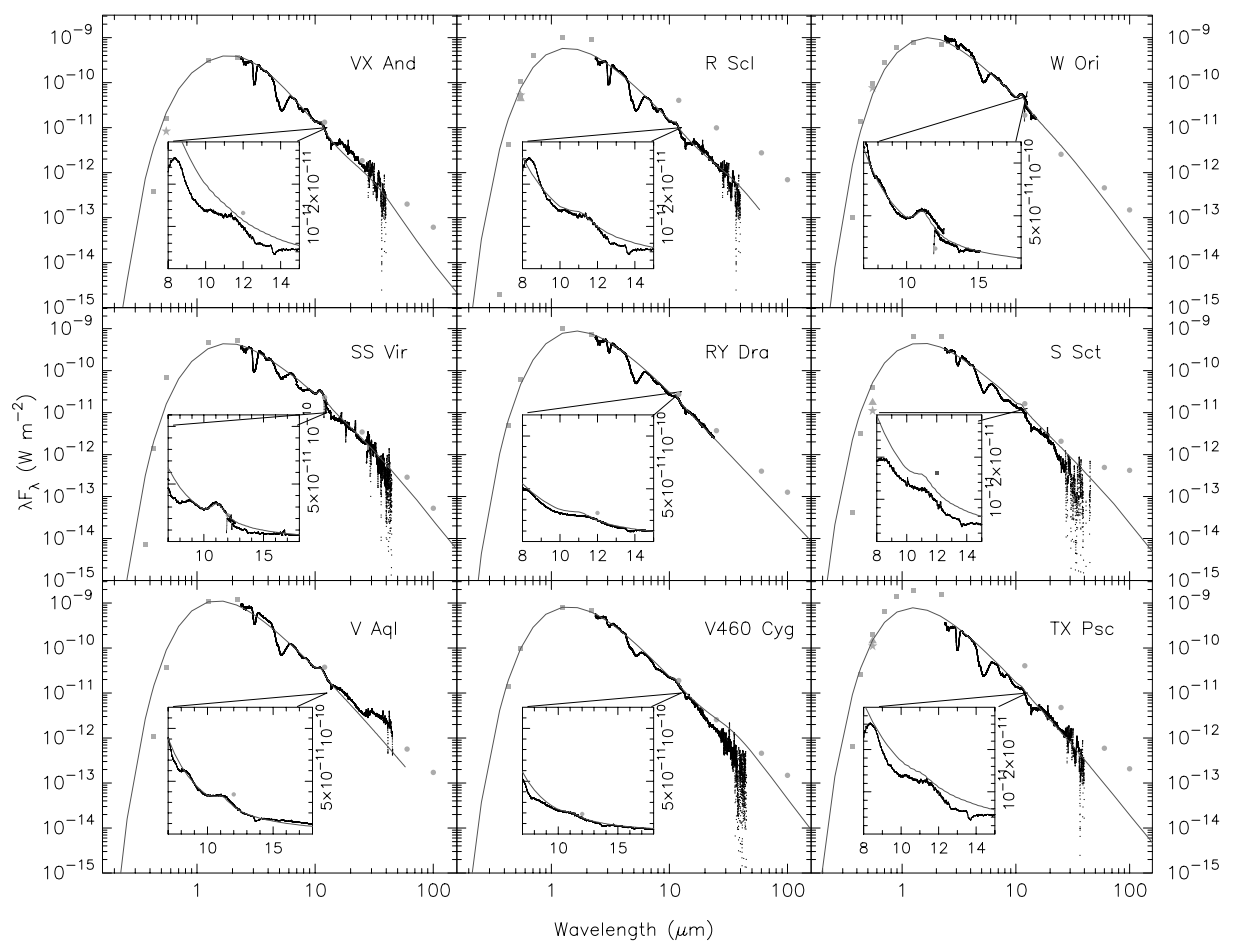

Figure 5.3: The photometrics bands, phtoemertics measurements and the ISO SWS spectra of our target sample. The BV and JHK photometric bands (displayed as squares), the IRAS 12, 25, 60 and $100 \mu \mathrm{m}$ photometric measurements (displayed as circles), the ISO SWS spectra (displayed as a dark dotted line), and and the modeled continuum (thick grey line). 


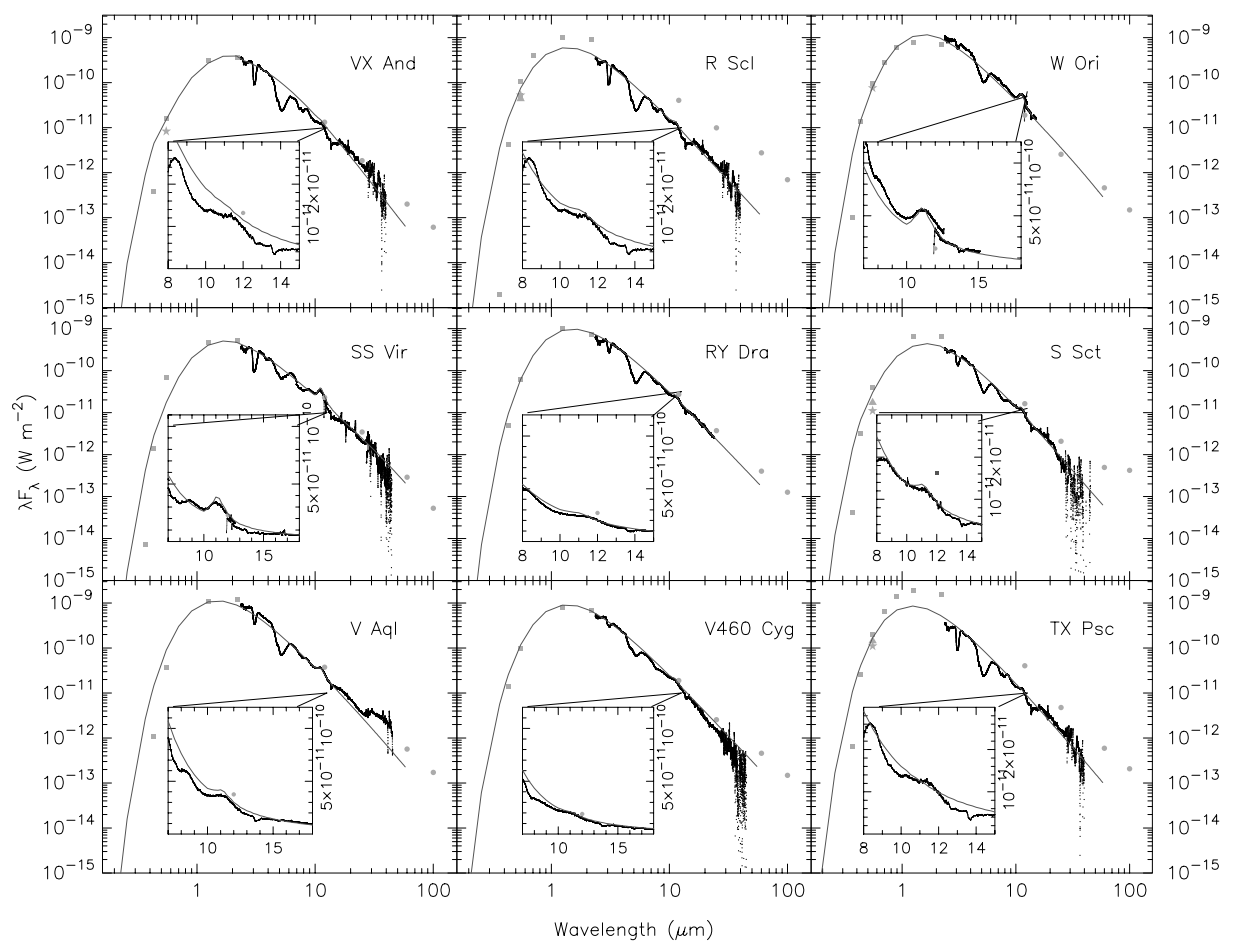

Figure 5.4: The photometrics bands, phtoemertics measurements and the ISO SWS spectra of our target sample. The BV and JHK photometric bands (displayed as squares), the IRAS 12, 25, 60 and $100 \mu \mathrm{m}$ photometric measurements (displayed as circles), the ISO SWS spectra (displayed as a dark dotted line), and and the modeled continuum (thick grey line). 


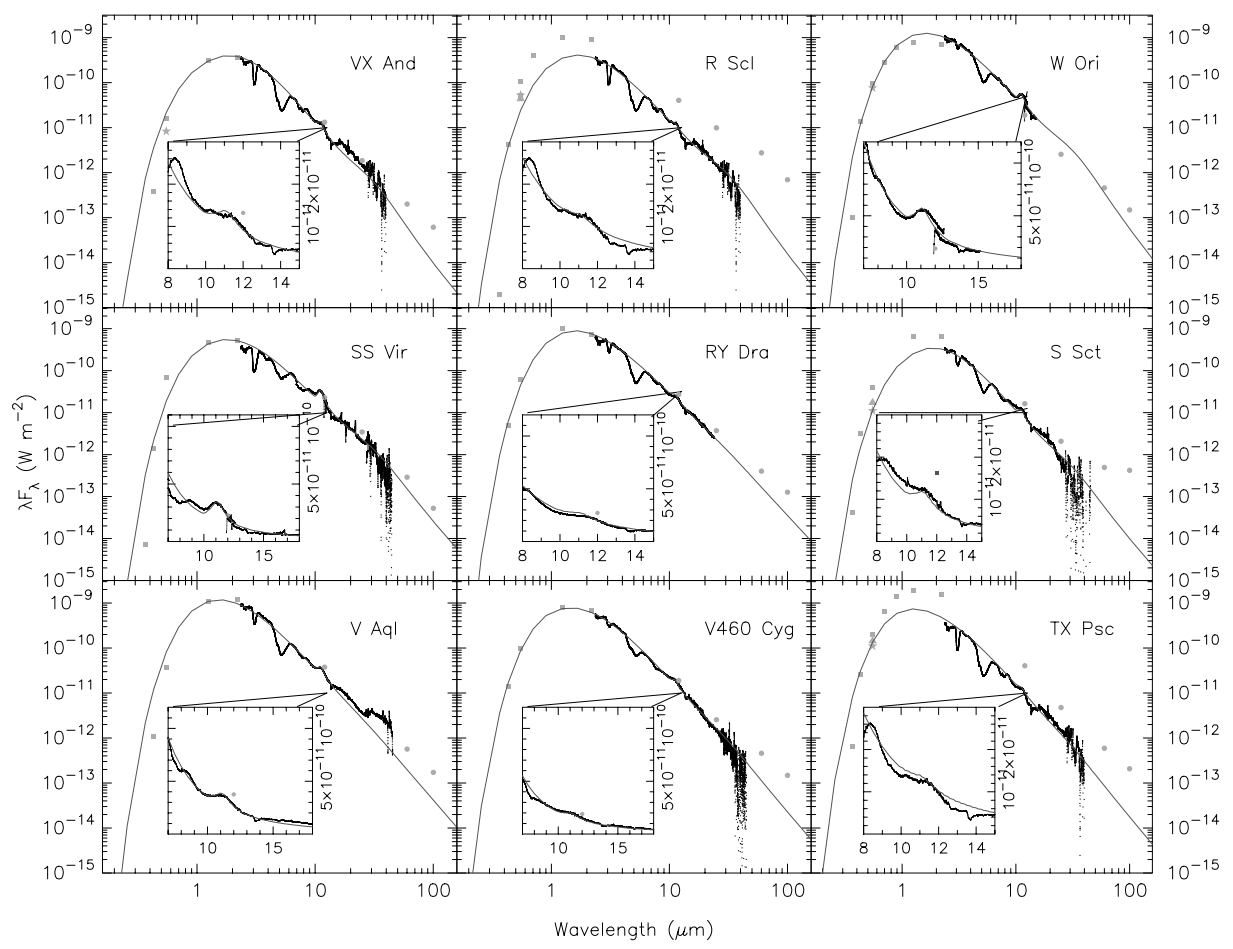

Figure 5.5: The photometrics bands, phtoemertics measurements and the ISO SWS spectra of our target sample. The BV and JHK photometric bands (displayed as squares), the IRAS 12, 25, 60 and $100 \mu \mathrm{m}$ photometric measurements (displayed as circles), the ISO SWS spectra (displayed as a dark dotted line), and and the modeled continuum (thick grey line). 


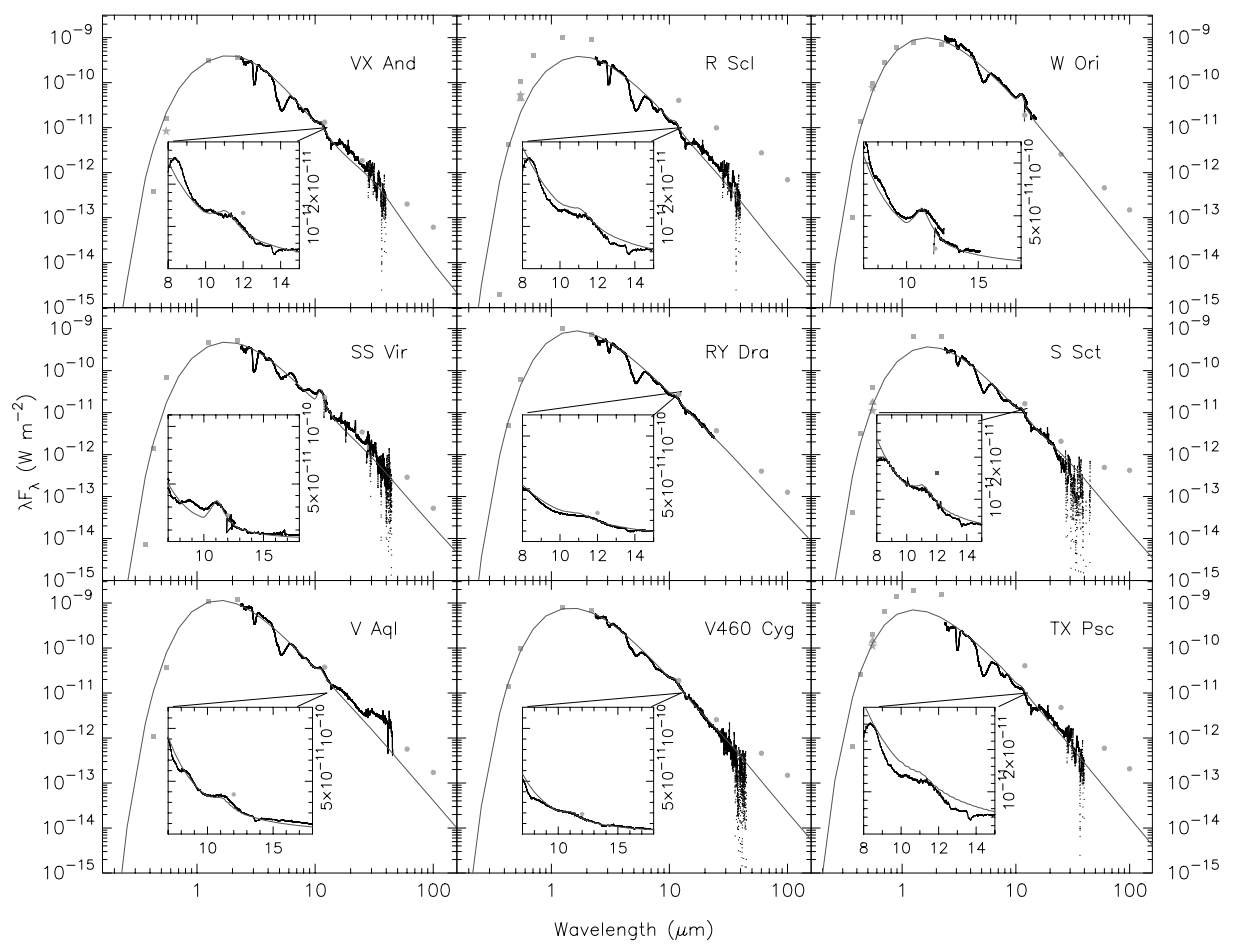

Figure 5.6: The photometrics bands, phtoemertics measurements and the ISO SWS spectra of our target sample. The BV and JHK photometric bands (displayed as squares), the IRAS 12, 25, 60 and $100 \mu \mathrm{m}$ photometric measurements (displayed as circles), the ISO SWS spectra (displayed as a dark dotted line), and and the modeled continuum (thick grey line). 


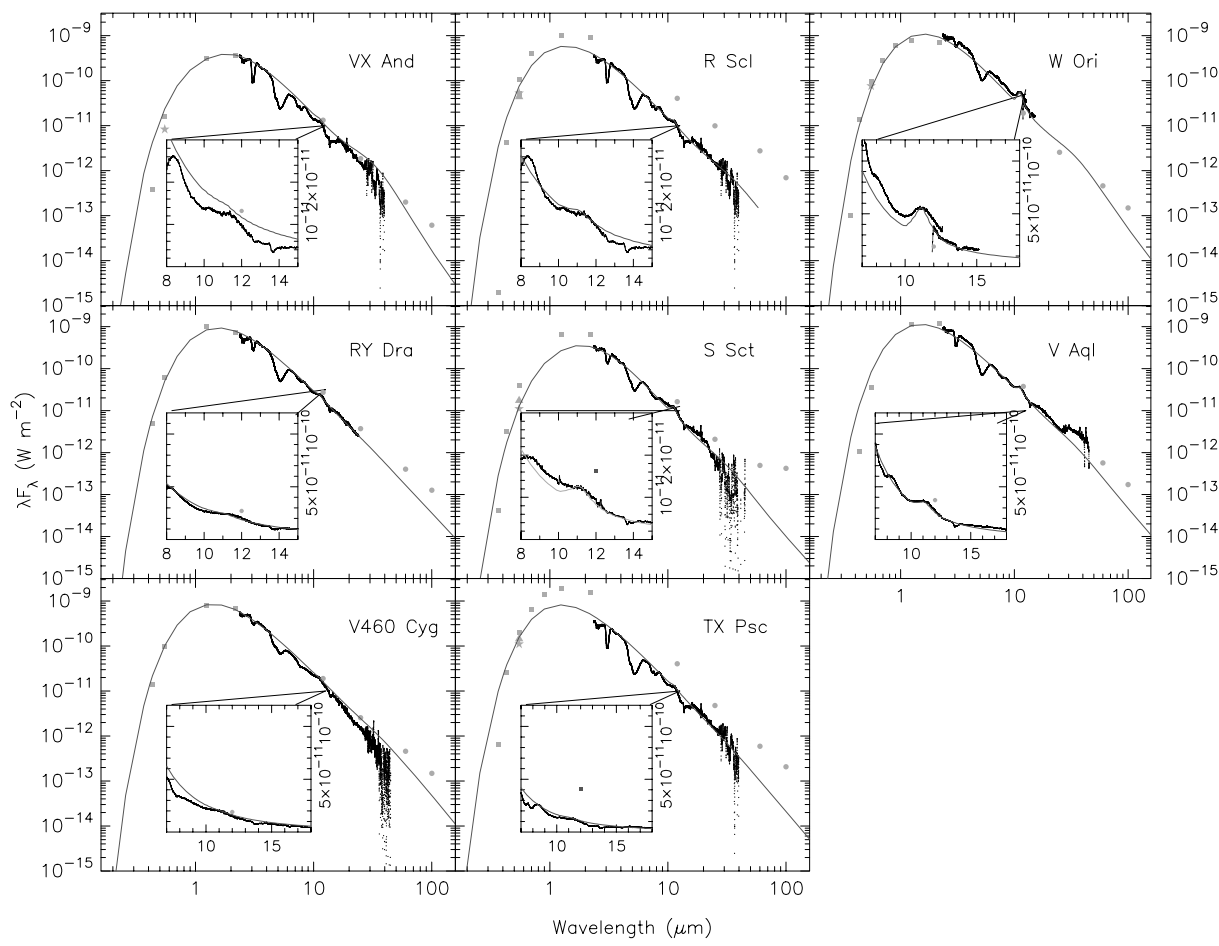

Figure 5.7: The photometrics bands, phtoemertics measurements and the ISO SWS spectra of our target sample. The BV and JHK photometric bands (displayed as squares), the IRAS 12, 25, 60 and $100 \mu \mathrm{m}$ photometric measurements (displayed as circles), the ISO SWS spectra (displayed as a dark dotted line), and and the modeled continuum (thick grey line). 


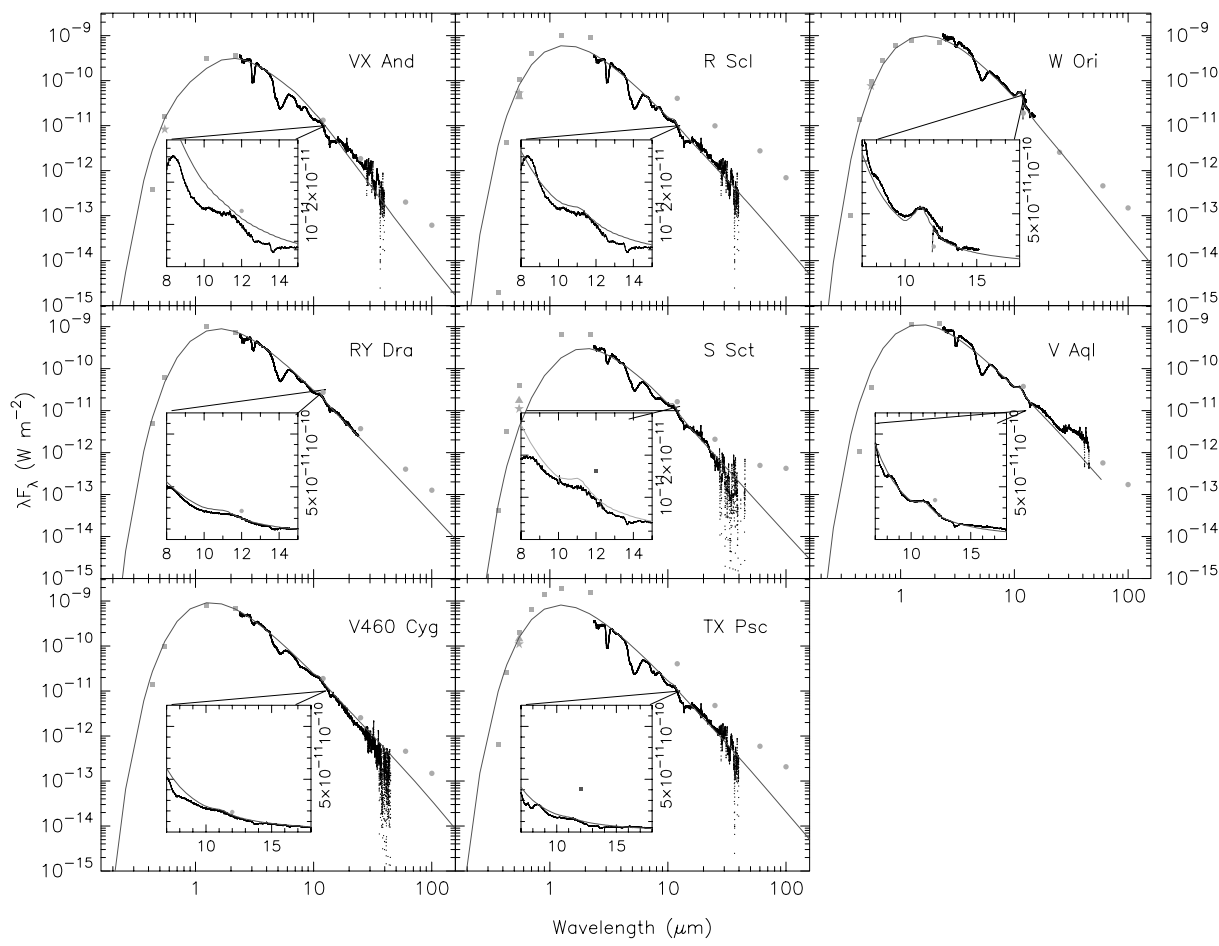

Figure 5.8: The photometrics bands, phtoemertics measurements and the ISO SWS spectra of our target sample. The BV and JHK photometric bands (displayed as squares), the IRAS 12, 25, 60 and $100 \mu \mathrm{m}$ photometric measurements (displayed as circles), the ISO SWS spectra (displayed as a dark dotted line), and and the modeled continuum (thick grey line). 
Table 5.4: Parameters for graphite single shell modeling.

\begin{tabular}{lcrrcc}
\hline Name & $\mathrm{T}_{\star}[\mathrm{K}]$ & $\mathrm{T}_{i n}[\mathrm{~K}]$ & $\xi$ & $\tau_{0.55 \mu m}$ & Graphite/SiC \\
\hline VX And & 3200 & 1600 & 100 & 2.145 & $99 / 1$ \\
R Scl & 2830 & 1750 & 10000 & 0.215 & $90 / 10$ \\
W Ori & 2680 & 1700 & 1000 & 1 & $74 / 26$ \\
SS Vir & 2560 & 1525 & 1000 & 1.216 & $79 / 21$ \\
RY Dra & 2500 & 1675 & 10 & 0.464 & $84 / 16$ \\
S Sct & 2895 & 1550 & 10 & 2.145 & $89 / 11$ \\
V Aql & 2610 & 1700 & 1000 & 1.0 & $80 / 20$ \\
V460 Cyg & 2845 & 1550 & 10000 & 0.464 & $95 / 5$ \\
TX Psc & 3010 & 1525 & 1000 & 0.1 & $90 / 10$ \\
\hline
\end{tabular}

$\mathrm{T}_{\star}$ is the star's effective temperature.

$\mathrm{T}_{i n}$ is the inner dust temperature, determined from the condensation temperature as described in $\S$ 4.4.1.

$\xi$ is the relative shell thickness $=R_{\text {out }} / R_{\text {in }}$. 
Table 5.5: Parameters for AmC single shell modeling.

\begin{tabular}{lrrrrr}
\hline Name & $\mathrm{T}_{\star}[\mathrm{K}]$ & $\mathrm{T}_{i n}[\mathrm{~K}]$ & $\xi$ & $\tau_{0.55 \mu m}$ & $\mathrm{AmC} / \mathrm{SiC}$ \\
\hline VX And & 3200 & 1600 & 15 & 2.145 & $98 / 2$ \\
R Scl & 2830 & 1750 & 10000 & 0.215 & $82 / 12$ \\
W Ori & 2680 & 1700 & 1000 & 1.0 & $80 / 20$ \\
SS Vir & 2560 & 1525 & 1000 & 1.216 & $80 / 20$ \\
RY Dra & 2500 & 1675 & 10 & 0.464 & $68 / 32$ \\
S Sct & 2895 & 1550 & 1000 & 2.145 & $87 / 13$ \\
V Aql & 2610 & 1700 & 10 & 1.0 & $70 / 30$ \\
V460 Cyg & 2845 & 1550 & 10000 & 0.215 & $90 / 10$ \\
TX Psc & 3010 & 1525 & 1000 & 0.1 & $88 / 12$ \\
\hline
\end{tabular}

$\mathrm{T}_{\star}$ is the star's effective temperature.

$\mathrm{T}_{i n}$ is the inner dust temperature, determined from the condensation temperature as described in $\S$ 4.4.1.

$\xi$ is the relative shell thickness $=R_{\text {out }} / R_{\text {in }}$. 
Table 5.6: Parameters for iron single shell modeling.

\begin{tabular}{lrrrrr}
\hline Name & $\mathrm{T}_{\star}[\mathrm{K}]$ & $\mathrm{T}_{i n}[\mathrm{~K}]$ & $\xi$ & $\tau_{0.55 \mu m}$ & Iron/SiC \\
\hline VX And & 3200 & 1600 & 15 & 2.145 & $99 / 1$ \\
R Scl & 2830 & 1750 & 10000 & 1.0 & $90 / 10$ \\
W Ori & 2680 & 1700 & 1000 & 10 & $90 / 10$ \\
SS Vir & 2560 & 1525 & 1000 & 5.0 & $70 / 30$ \\
RY Dra & 2500 & 1675 & 10 & 1.0 & $60 / 40$ \\
S Sct & 2895 & 1550 & 1000 & 2.145 & $85 / 15$ \\
V Aql & 2610 & 1700 & 10 & 0.464 & $70 / 30$ \\
V460 Cyg & 2845 & 1550 & 10000 & 1.0 & $95 / 05$ \\
TX Psc & 3010 & 1525 & 1000 & 0.1 & $95 / 05$ \\
\hline
\end{tabular}

$\mathrm{T}_{\star}$ is the star's effective temperature.

$\mathrm{T}_{i n}$ is the inner dust temperature, determined from the condensation temperature as described in $\S$ 4.4.1.

$\xi$ is the relative shell thickness $=R_{\text {out }} / R_{\text {in }}$. 
Table 5.7: Parameters for 2-shell Graphite modeling.

\begin{tabular}{lcccccc}
\hline Name & $\mathrm{T}_{\text {in }}[\mathrm{K}]$ & $\mathrm{T}_{\text {out }}[\mathrm{K}]$ & $\xi_{\text {out }}$ & $\tau_{0.55 \mu m i n}$ & $\tau_{0.55 \mu m o u t}$ & Graphite $/ \mathrm{SiC}\left(2^{\text {nd }}\right.$ shell $)$ \\
\hline VX And & 1600 & 1175 & 10 & 2.145 & 0.1 & $10 / 90$ \\
R Scl & 1750 & 1250 & 100 & 0.464 & 0.464 & $95 / 5$ \\
W Ori & 1700 & 1175 & 10 & 0.464 & 0.464 & $85 / 15$ \\
SS Vir & 1525 & 1175 & 10 & 1.0 & 0.464 & $65 / 35$ \\
RY Dra & 1675 & 1250 & 10 & 0.464 & 0.1 & $55 / 45$ \\
S Sct & 1650 & 1225 & 10 & 2.145 & 0.1 & $05 / 95$ \\
V Aql & 1700 & 1200 & 10 & 0.464 & 0.1 & $25 / 75$ \\
V460 Cyg & 1550 & 1200 & 10 & 0.464 & 0.1 & $65 / 35$ \\
TX Psc & 1525 & 1175 & 10 & 1.0 & 0.464 & $85 / 15$ \\
\hline
\end{tabular}

$\mathrm{T}_{\star}$, the star's effective temperature can be found in Table 5.4.

$\mathrm{T}_{i n}$ is the inner dust temperature, determined from the condensation temper-

ature as described in $\S 4.4 .1$.

$\xi$ is the relative shell thickness $=R_{\text {out }} / R_{\text {in }}$.

$\xi_{\text {inner }}$ was 10 for all the inner shells, the inner dust shell was solid graphite. 
Table 5.8: Parameters for 2-shell AmC modeling.

\begin{tabular}{lcccccc}
\hline Name & $\mathrm{T}_{\text {in }}[\mathrm{K}]$ & $\mathrm{T}_{\text {out }}[\mathrm{K}]$ & $\xi_{\text {out }}$ & $\tau_{0.55 \mu m i n}$ & $\tau_{0.55 \mu m o u t}$ & $\mathrm{AmC} / \mathrm{SiC}\left(2^{\text {nd }}\right.$ shell $)$ \\
\hline VX And & 1600 & 1175 & 10 & 2.145 & 0.1 & $10 / 90$ \\
R Scl & 1750 & 1250 & 10 & 0.464 & 0.464 & $85 / 15$ \\
W Ori & 1700 & 1175 & 10 & 0.464 & 0.464 & $60 / 40$ \\
SS Vir & 1525 & 1175 & 10 & 1.0 & 0.464 & $80 / 20$ \\
RY Dra & 1675 & 1250 & 10 & 0.464 & 0.1 & $50 / 50$ \\
S Sct & 1650 & 1225 & 10 & 2.145 & 0.1 & $50 / 50$ \\
V Aql & 1700 & 1200 & 10 & 0.464 & 0.1 & $45 / 55$ \\
V460 Cyg & 1550 & 1200 & 10 & 0.464 & 0.1 & $65 / 35$ \\
TX Psc & 1525 & 1175 & 10 & 1.0 & 0.464 & $85 / 15$ \\
\hline
\end{tabular}

$\mathrm{T}_{\star}$, the star's effective temperature can be found in Table 5.4.

$\mathrm{T}_{i n}$ is the inner dust temperature, determined from the condensation temperature as described in $\S 4.4 .1$.

$\xi$ is the relative shell thickness $=R_{\text {out }} / R_{\text {in }}$.

$\xi_{\text {inner }}$ was 10 for all the inner shells, the inner dust shell was solid graphite. 
Table 5.9: Parameters for graphite/Fe single shell modeling.

\begin{tabular}{lrrrrc}
\hline Name & $\mathrm{T}_{\star}[\mathrm{K}]$ & $\mathrm{T}_{\text {in }}[\mathrm{K}]$ & $\xi$ & $\tau_{0.55 \mu m}$ & Graphite/Iron/SiC \\
\hline VX And & 3200 & 1600 & 100 & 2.145 & $95 / 4 / 01$ \\
R Scl & 2830 & 1750 & 1000 & 0.215 & $49 / 40 / 11$ \\
W Ori & 2680 & 1700 & 1000 & 1.0 & $74 / 0 / 26$ \\
RY Dra & 2500 & 1675 & 10 & 0.464 & $74 / 10 / 16$ \\
S Sct & 2895 & 1550 & 10 & 2.145 & $47 / 45 / 08$ \\
V Aql & 2610 & 1700 & 1000 & 0.464 & $53 / 30 / 17$ \\
V460 Cyg & 2845 & 1550 & 10000 & 0.464 & $23 / 76 / 01$ \\
TX Psc & 3010 & 1525 & 1000 & 0.1 & $18 / 72 / 10$ \\
\hline
\end{tabular}

$T_{\star}$ is the star's effective temperature.

$\mathrm{T}_{i n}$ is the inner dust temperature, determined from the condensation temperature as described in $\S$ 4.4.1.

$\xi$ is the relative shell thickness $=R_{\text {out }} / R_{\text {in }}$. 
Table 5.10: Parameters for AmC/Fe single shell modeling.

\begin{tabular}{lccrcc} 
Name & $\mathrm{T}_{\star}[\mathrm{K}]$ & $\mathrm{T}_{i n}[\mathrm{~K}]$ & $\xi$ & $\tau_{0.55 \mu m}$ & $\mathrm{AmC} / \mathrm{Iron} / \mathrm{SiC}$ \\
\hline VX And & 3200 & 1600 & 100 & 2.145 & $63 / 35 / 02$ \\
R Scl & 2830 & 1750 & 1000 & 0.215 & $44 / 37 / 19$ \\
W Ori & 2680 & 1700 & 1000 & 1.0 & $80 / 0 / 20$ \\
RY Dra & 2500 & 1675 & 10 & 0.464 & $74 / 10 / 16$ \\
S Sct & 2895 & 1550 & 10 & 2.145 & $60 / 25 / 15$ \\
V Aql & 2610 & 1700 & 1000 & 0.464 & $47 / 23 / 30$ \\
V460 Cyg & 2845 & 1550 & 10000 & 0.464 & $22 / 68 / 10$ \\
TX Psc & 3010 & 1525 & 1000 & 0.1 & $18 / 72 / 10$ \\
\hline
\end{tabular}

$T_{\star}$ is the star's effective temperature.

$\mathrm{T}_{i n}$ is the inner dust temperature, determined from the condensation temperature as described in $\S$ 4.4.1.

$\xi$ is the relative shell thickness $=R_{\text {out }} / R_{\text {in }}$. 
Having found good model fits to our sample stars, we sought correlations between model parameters and the various collated parameters listed in Tables 5.2-5.10 using simple least squares fits. We followed the work of Thompson et al. (2006) and Messenger et al. (2013) in only taking correlations to be significant if the correlation coefficient is, $\mathrm{R} \geq 0.7$; i.e. the determination coefficient, $R^{2}$, is $\geq 0.5$. The resulting data correlations (or lack thereof) are plotted in Figures 5.9-5.30 and found Tables 5.11-5.20.

\subsubsection{Correlations Between Collated and Model Parameters}

For the collated data (i.e. not model parameters) there existed some significant correlations: (1) for dust formation temperature and the change of temperature from the formation of graphite grains to the formation of $\mathrm{SiC}$ grains (see Table 5.11), and (2) $\mathrm{C} / \mathrm{O}$ and the change of temperature from the formation of graphite grains to the formation of $\mathrm{SiC}$ grains (see Table 5.11; which is based on the collated parameters and provides the input parameters $\mathrm{T}_{i n}$ for all models, see $\S$ 4.4.1).

\subsubsection{Correlations Between Model Parameters}

While there was no significant correlations for any of our single shell graphite, AmC and iron-bearing models or 2-shell graphite and $\mathrm{AmC}$ models, there were signification correlations with our single shell graphite/Fe and $\mathrm{AmC} / \mathrm{Fe}$ models and the abundance of $\mathrm{SiC}$ grains between the different models. For the single-shell modeling with graphite and iron grains and $\mathrm{AmC}$ and iron grains there were significant correlations between: 


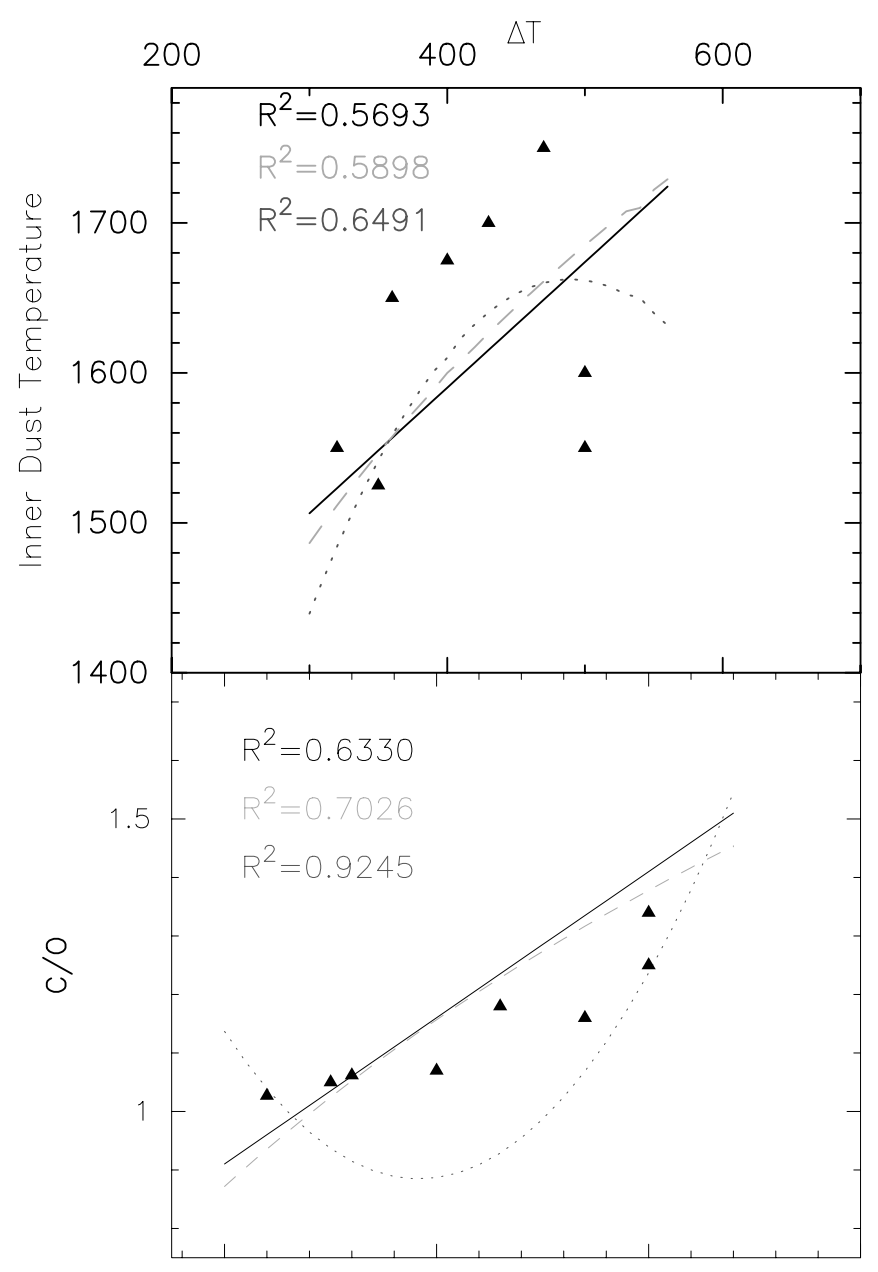

Figure 5.9: The correlations between the inner dust temperature and the change of temperature between the formation of graphite to $\mathrm{SiC}$ grains and the $\mathrm{C} / \mathrm{O}$ ratio and change of temperature between the formation of graphite to $\mathrm{SiC}$ grains for the collated parameters. 
Table 5.11: Correlations amongst collated parameters

\begin{tabular}{lcccc} 
& $\mathrm{T}_{i n}$ & $\mathrm{C} / \mathrm{O}$ & $\mathrm{Fe} / \mathrm{H}$ & $\mathrm{V}_{\text {exp }}$ \\
\hline \hline \multicolumn{5}{c}{ Linear } \\
\hline $\mathrm{T}_{\star}$ & 0.0805 & 0.2503 & 0.0151 & 0.2852 \\
$\mathrm{~T}_{\text {in }}$ & - & 0.0913 & 0.0285 & 0.0057 \\
$\mathrm{C} / \mathrm{O}$ & 0.8750 & - & 0.0637 & 0.0860 \\
$\mathrm{Fe} / \mathrm{H}$ & 0.0285 & 0.0637 & - & 0.1240 \\
\hline & \multicolumn{5}{c}{ Log } \\
\hline $\mathrm{T}_{\star}$ & 0.0805 & 0.2112 & 0.0482 & 0.3416 \\
$\mathrm{~T}_{\text {in }}$ & - & 0.2783 & 0.0261 & 0.1463 \\
$\mathrm{C} / \mathrm{O}$ & 0.2783 & - & 0.0677 & 0.0918 \\
$\mathrm{Fe} / \mathrm{H}$ & 0.0261 & 0.0918 & - & 0.1397 \\
\hline & \multicolumn{5}{c}{ Poly (power 2$)$} \\
\hline $\mathrm{T} \mathrm{T}_{\star}$ & 0.127 & 0.458 & 0.0151 & 0.4478 \\
$\mathrm{~T}_{\text {in }}$ & - & 0.1438 & 0.1931 & 0.0008 \\
$\mathrm{C} / \mathrm{O}$ & .9474 & - & 0.0772 & 0.1049 \\
$\mathrm{Fe} / \mathrm{H}$ & 0.1931 & 0.0772 & - & 0.1940 \\
\hline
\end{tabular}


Table 5.11: Continued. Correlations amongst collated parameters

\begin{tabular}{|c|c|c|c|c|}
\hline & $\mathrm{T}_{i n}$ & $\mathrm{C} / \mathrm{O}$ & $\mathrm{Fe} / \mathrm{H}$ & $\mathrm{v}_{\text {exp }}$ \\
\hline \multicolumn{5}{|c|}{ Linear } \\
\hline $\mathrm{v}_{\text {exp }}$ & 0.0057 & 0.0508 & 0.1240 & - \\
\hline$\dot{M}$ & 0.0087 & 0.0300 & 0.0682 & 0.3000 \\
\hline $\mathrm{L}$ & 0.0228 & 0.0499 & 0.0012 & 0.0209 \\
\hline${ }^{12} C /{ }^{13} C$ & 0.0866 & 0.0499 & 0.0012 & 0.0209 \\
\hline $\mathrm{g} / \mathrm{d}$ & 0.0022 & 0.0390 & 0.0858 & 0.0427 \\
\hline \multicolumn{5}{|c|}{$\log$} \\
\hline $\mathrm{v}_{\text {exp }}$ & 0.1463 & 0.0321 & 0.2273 & - \\
\hline$\dot{M}$ & 0.0080 & 0.0262 & 0.2273 & 0.0321 \\
\hline $\mathrm{L}$ & 0.0364 & 0.0012 & 0.0397 & 0.0191 \\
\hline${ }^{12} C /{ }^{13} C$ & 0.0491 & 0.0652 & 0.1922 & 0.0098 \\
\hline $\mathrm{g} / \mathrm{d}$ & 0.2485 & 0.0086 & 0.0858 & 0.0175 \\
\hline \multicolumn{5}{|c|}{ Poly (power 2) } \\
\hline $\mathrm{V}_{e x p}$ & 0.0008 & 0.1049 & 0.1940 & - \\
\hline$\dot{M}$ & 0.1002 & 0.1527 & 0.0682 & 0.1527 \\
\hline $\mathrm{L}$ & 0.0229 & 0.0721 & 0.0180 & 0.0511 \\
\hline${ }^{12} C /{ }^{13} C$ & 0.1390 & 0.0776 & 0.0334 & 0.0433 \\
\hline $\mathrm{g} / \mathrm{d}$ & 0.4427 & 0.0088 & 0.0197 & 0.0469 \\
\hline
\end{tabular}


Table 5.11: Continued. Correlations amongst collated parameters $\dot{M} \quad \mathrm{~L} \quad{ }^{12} C /{ }^{13} C \quad$ g/d $\quad \Delta \mathrm{T}: \mathrm{SiC} \rightarrow$ Graphite

\begin{tabular}{lccccc}
\hline \hline \multicolumn{5}{c}{ Linear } \\
\hline $\mathrm{T}_{\star}$ & 0.0007 & 0.0445 & 0.0185 & 0.2741 & 0.0206 \\
$\mathrm{~T}_{\text {in }}$ & 0.3965 & 0.0228 & 0.0866 & 0.0022 & $\mathbf{0 . 5 6 9 3}$ \\
$\mathrm{C} / \mathrm{O}$ & 0.0508 & 0.0134 & 0.0499 & 0.0390 & $\mathbf{0 . 6 3 3 0}$ \\
$\mathrm{Fe} / \mathrm{H}$ & 0.0153 & 0.0242 & 0.0012 & 0.0858 & 0.1092 \\
\hline & & & \multicolumn{2}{c}{ Log } & \\
\hline $\mathrm{T}_{\star}$ & 0.0934 & 0.0609 & 0.0738 & 0.2485 & 0.0097 \\
$\mathrm{~T}_{\text {in }}$ & 0.4150 & 0.0364 & 0.0491 & 0.0053 & $\mathbf{0 . 5 8 9 8}$ \\
$\mathrm{C} / \mathrm{O}$ & 0.0321 & 0.0012 & 0.0652 & 0.0086 & $\mathbf{0 . 7 0 2 6}$ \\
$\mathrm{Fe} / \mathrm{H}$ & 0.2273 & 0.0397 & 0.1922 & 0.0858 & 0.1609 \\
\hline & & & Poly (power 2$)$ & \\
\hline $\mathrm{T}_{\star}$ & 0.2999 & 0.0507 & 0.0263 & 0.4427 & 0.4993 \\
$\mathrm{~T}_{\text {in }}$ & 0.5484 & 0.0229 & 0.1390 & 0.0088 & $\mathbf{0 . 6 4 9 1}$ \\
$\mathrm{C} / \mathrm{O}$ & 0.0511 & 0.0721 & 0.0776 & 0.0197 & $\mathbf{0 . 9 2 4 5}$ \\
$\mathrm{Fe} / \mathrm{H}$ & 0.0682 & 0.018 & 0.0334 & 0.0469 & 0.0971 \\
\hline
\end{tabular}


Table 5.11: Contindued. Correlations amongst collated parameters

\begin{tabular}{|c|c|c|c|c|c|}
\hline & $\dot{M}$ & $\mathrm{~L}$ & ${ }^{12} C /{ }^{13} C$ & $\mathrm{~g} / \mathrm{d}$ & $\Delta \mathrm{T}: \mathrm{SiC} \rightarrow$ Graphite \\
\hline \multicolumn{6}{|c|}{ Linear } \\
\hline $\mathrm{v}_{e x p}$ & 0.0300 & 0.0076 & 0.0209 & 0.0427 & 0.0682 \\
\hline$\dot{M}$ & - & 0.1104 & 0.0726 & 0.0600 & 0.1793 \\
\hline $\mathrm{L}$ & 0.0726 & - & 0.1019 & 0.0225 & 0.0367 \\
\hline${ }^{12} C /{ }^{13} C$ & 0.0600 & 0.1019 & - & 0.0103 & 0.1025 \\
\hline$g / d$ & 0.0600 & 0.0225 & 0.0103 & - & 0.2634 \\
\hline \multicolumn{6}{|c|}{$\log$} \\
\hline $\mathrm{V}_{\text {exp }}$ & 0.0262 & 0.0191 & 0.0098 & 0.0175 & 0.0655 \\
\hline$\dot{M}$ & - & 0.0747 & 0.0221 & 0.0334 & 0.1682 \\
\hline $\mathrm{L}$ & 0.00747 & - & 0.0037 & 0.0028 & 0.0535 \\
\hline${ }^{12} C /{ }^{13} C$ & 0.0221 & 0.0037 & - & 0.0271 & 0.0996 \\
\hline$g / d$ & 0.0334 & 0.0028 & 0.0103 & - & 0.2407 \\
\hline \multicolumn{6}{|c|}{ Poly (power 2) } \\
\hline $\mathrm{V}_{\text {exp }}$ & 0.1527 & 0.0511 & 0.0433 & 0.0911 & 0.0751 \\
\hline$\dot{M}$ & - & 0.1104 & 0.0726 & 0.0600 & 0.1793 \\
\hline $\mathrm{L}$ & 0.1104 & - & 0.1284 & 0.0518 & 0.3793 \\
\hline${ }^{12} C /{ }^{13} C$ & 0.0726 & 0.1284 & - & 0.0477 & 0.1031 \\
\hline $\mathrm{g} / \mathrm{d}$ & 0.0911 & 0.0600 & 0.0518 & - & 0.4129 \\
\hline
\end{tabular}


Table 5.12: Correlations between modeled and collated parameters for single shell graphite

\begin{tabular}{|c|c|c|c|c|c|}
\hline & $\mathrm{T}_{i n}$ & $\tau_{0.55 \mu m}$ & Graphite & $\mathrm{SiC}$ & $\Delta \mathrm{T}$ \\
\hline \multicolumn{6}{|c|}{ Linear } \\
\hline$\xi$ & 0.0363 & 0.2521 & 0.1514 & 0.1514 & 0.0002 \\
\hline$\tau_{0.55 \mu m}$ & 0.0751 & - & 0.0448 & 0.0488 & 0.0165 \\
\hline Graphite & 0.0593 & 0.0448 & - & 1.000 & 0.0088 \\
\hline $\mathrm{SiC}$ & 0.0593 & 0.0448 & 1.000 & - & 0.0088 \\
\hline \multicolumn{6}{|c|}{$\log$} \\
\hline$\xi$ & 0.0328 & 0.2009 & 0.1472 & 0.1472 & 0.0443 \\
\hline$\tau_{0.55 \mu m}$ & 0.0695 & - & 0.0216 & 0.0216 & 0.0219 \\
\hline Graphite & 0.0613 & 0.0216 & - & 1.000 & 0.0035 \\
\hline $\mathrm{SiC}$ & 0.0613 & 0.0216 & 1.000 & - & 0.0035 \\
\hline \multicolumn{6}{|c|}{ Poly (power 2) } \\
\hline$\xi$ & 0.3308 & 0.2637 & 0.1527 & 0.1527 & 0.0106 \\
\hline$\tau_{0.55 \mu m}$ & 0.4107 & - & 0.3694 & 0.3694 & 0.3468 \\
\hline Graphite & 0.1313 & 0.3694 & - & 1.000 & 0.3063 \\
\hline $\mathrm{SiC}$ & 0.1313 & 0.3694 & 1.000 & - & 0.3063 \\
\hline
\end{tabular}


Table 5.12: Continued. Correlations between modeled and collated parameters for single shell graphite

\begin{tabular}{|c|c|c|c|c|c|c|c|c|}
\hline & $\mathrm{T}_{\star}$ & $\mathrm{C} / \mathrm{O}$ & $\mathrm{Fe} / \mathrm{H}$ & $\mathrm{V}_{e x p}$ & $\dot{M}$ & $\mathrm{~L}$ & ${ }^{12} \mathrm{C} /{ }^{13} \mathrm{C}$ & $\mathrm{g} / \mathrm{d}$ \\
\hline \multicolumn{9}{|c|}{ Linear } \\
\hline$\xi$ & 0.0348 & 0.1181 & 0.0198 & 0.1099 & 0.4009 & 0.0015 & 0.4367 & 0.0930 \\
\hline$\tau_{0.55 \mu m}$ & 0.000 & 0.1359 & 0.0762 & 0.0069 & 0.1518 & 0.0315 & 0.0603 & 0.2292 \\
\hline Graphite & 0.4706 & 0.0593 & 0.000 & 0.1363 & 0.1135 & 0.0158 & 0.000 & 0.1199 \\
\hline $\mathrm{SiC}$ & 0.4706 & 0.000 & 0.1363 & 0.1135 & 0.0158 & 0.000 & 0.1199 & 0.0533 \\
\hline \multicolumn{9}{|c|}{$\log$} \\
\hline$\xi$ & 0.0435 & 0.1046 & 0.0106 & 0.0016 & 0.0998 & 0.2002 & 0.0055 & 0.3397 \\
\hline$\tau_{0.55 \mu m}$ & 0.0005 & 0.1263 & 0.0391 & 0.0016 & 0.2721 & 0.0024 & 0.0724 & 0.2432 \\
\hline Graphite & 0.3845 & 0.3561 & 0.4659 & 0.1635 & 0.0142 & 0.0009 & 0.0936 & 0.0003 \\
\hline $\mathrm{SiC}$ & 0.3845 & 0.3561 & 0.4659 & 0.1635 & 0.0142 & 0.0009 & 0.0936 & 0.0003 \\
\hline \multicolumn{9}{|c|}{ Poly (power 2) } \\
\hline$\xi$ & 0.2478 & 0.4155 & 0.0244 & 0.1325 & 0.4532 & 0.0021 & 0.4434 & 0.0943 \\
\hline$\tau_{0.55 \mu m}$ & 0.3800 & 0.2371 & 0.1726 & 0.0366 & 0.2036 & 0.4659 & 0.1112 & 0.0000 \\
\hline Graphite & 0.4584 & 0.2896 & 0.1572 & 0.1180 & 0.1708 & 0.0377 & 0.1319 & 0.4950 \\
\hline $\mathrm{SiC}$ & 0.4584 & 0.2896 & 0.1572 & 0.1180 & 0.1708 & 0.0377 & 0.1319 & 0.4950 \\
\hline
\end{tabular}


Table 5.13: Correlations between modeled and collated parameters for single shell graphite

\begin{tabular}{|c|c|c|c|c|c|}
\hline & $\mathrm{T}_{i n}$ & $\tau_{0.55 \mu m}$ & $\mathrm{AmC}$ & $\mathrm{SiC}$ & $\Delta \mathrm{T}$ \\
\hline \multicolumn{6}{|c|}{ Linear } \\
\hline$\xi$ & 0.0418 & 0.0001 & 0.0534 & 0.0534 & 0.0030 \\
\hline$\tau_{0.55 \mu m}$ & 0.0703 & - & 0.0874 & 0.0874 & 0.3531 \\
\hline $\mathrm{AmC}$ & 0.2056 & 0.0874 & - & 1.000 & 0.0112 \\
\hline $\mathrm{SiC}$ & 0.2056 & 0.0874 & 1.000 & - & 0.0112 \\
\hline \multicolumn{6}{|c|}{$\log$} \\
\hline$\xi$ & 0.0379 & 0.0086 & 0.0641 & 0.0641 & 0.0028 \\
\hline$\tau_{0.55 \mu m}$ & 0.0696 & - & 0.0871 & 0.0871 & 0.3542 \\
\hline $\mathrm{AmC}$ & 0.2076 & 0.0871 & - & 1.000 & 0.0183 \\
\hline $\mathrm{SiC}$ & 0.2076 & 0.0871 & 1.000 & - & 0.0183 \\
\hline \multicolumn{6}{|c|}{ Poly (power 2) } \\
\hline$\xi$ & 0.3463 & 0.1574 & 0.2059 & 0.2059 & 0.0597 \\
\hline$\tau_{0.55 \mu m}$ & 0.778 & - & 0.0881 & 0.0881 & 0.3531 \\
\hline $\mathrm{AmC}$ & 0.2255 & 0.0881 & - & 1.000 & 0.2932 \\
\hline $\mathrm{SiC}$ & 0.2255 & 0.0881 & 1.000 & - & 0.2932 \\
\hline
\end{tabular}


Table 5.13: Continued. Correlations between modeled and collated parameters for single shell AmC

\begin{tabular}{lcccccccc}
\hline & $\mathrm{T}_{\star}$ & $\mathrm{C} / \mathrm{O}$ & $\mathrm{Fe} / \mathrm{H}$ & $\mathrm{v}_{\text {exp }}$ & $\dot{M}$ & $\mathrm{~L}$ & ${ }^{12} C /{ }^{13} C$ & $\mathrm{~g} / \mathrm{d}$ \\
\hline$\xi$ & 0.0062 & 0.0089 & 0.0637 & 0.0641 & 0.3216 & 0.0020 & 0.4283 & 0.1002 \\
$\tau_{0.55 \mu m}$ & 0.0129 & 0.1254 & $\mathbf{0 . 7 3 1 3}$ & 0.3169 & 0.3695 & 0.1859 & 0.3652 & \\
$\mathrm{AmC}$ & 0.3947 & 0.2880 & 0.1912 & 0.1130 & 0.0070 & 0.0297 & 0.0838 & 0.3776 \\
$\mathrm{SiC}$ & 0.3947 & 0.2880 & 0.1912 & 0.1130 & 0.0070 & 0.0297 & 0.0838 & 0.3776 \\
\hline & & & & Log & & & & \\
\hline$\xi$ & 0.0102 & 0.0059 & 0.0677 & 0.0417 & 0.0918 & 0.0098 & 0.3067 & 0.0390 \\
$\tau_{0.55 \mu m}$ & 0.0113 & 0.3400 & 0.3470 & 0.4439 & 0.2303 & 0.3908 & 0.1233 & 0.3541 \\
$\mathrm{AmC}$ & 0.3882 & 0.2765 & 0.2039 & 0.1336 & 0.1050 & 0.1142 & 0.1006 & 0.3678 \\
$\mathrm{SiC}$ & 0.3882 & 0.0834 & 0.2765 & 0.1336 & 0.1050 & 0.1142 & 0.1006 & 0.3678 \\
\hline & & & & Poly (power 2$)$ & & & \\
\hline$\xi$ & 0.3439 & 0.0661 & 0.0772 & 0.4533 & 0.4755 & 0.0108 & 0.4554 & 0.1010 \\
$\tau_{0.55 \mu m}$ & 0.0597 & 0.3446 & 0.4835 & $\mathbf{0 . 8 4 9 8}$ & 0.3657 & 0.4219 & 0.2101 & 0.3654 \\
$\mathrm{AmC}$ & 0.4153 & 0.3598 & 0.2783 & 0.2911 & 0.0783 & 0.0965 & 0.1183 & 0.3964 \\
$\mathrm{SiC}$ & 0.4153 & 0.3598 & 0.2783 & 0.2911 & 0.0783 & 0.0965 & 0.1183 & 0.3964 \\
\hline
\end{tabular}


Table 5.14: Correlations between modeled and collated parameters for single shell iron

\begin{tabular}{|c|c|c|c|c|c|}
\hline & $\mathrm{T}_{i n}$ & $\tau_{0.55 \mu m}$ & Iron & $\mathrm{SiC}$ & $\Delta \mathrm{T}: \mathrm{SiC} \rightarrow$ Graphite \\
\hline \multicolumn{6}{|c|}{ Linear } \\
\hline$\xi$ & 0.0418 & 0.0508 & 0.1523 & 0.1523 & 0.0030 \\
\hline$\tau_{0.55 \mu m}$ & 0.0349 & - & 0.0024 & 0.0024 & 0.0112 \\
\hline Iron & 0.0218 & 0.0024 & - & 1.000 & 0.0007 \\
\hline $\mathrm{SiC}$ & 0.0218 & 0.0024 & 1.000 & - & 0.0007 \\
\hline \multicolumn{6}{|c|}{$\log$} \\
\hline$\xi$ & 0.0379 & 0.0078 & 0.1569 & 0.1569 & 0.0028 \\
\hline$\tau_{0.55 \mu m}$ & 0.1135 & 0.0349 & - & 0.0045 & 0.0152 \\
\hline Iron & 0.0230 & 0.0045 & - & 1.000 & 0.000 \\
\hline $\mathrm{SiC}$ & 0.0230 & 0.0045 & 1.000 & - & 0.000 \\
\hline \multicolumn{6}{|c|}{ Poly (power 2) } \\
\hline$\xi$ & 0.3463 & 0.0570 & 0.1718 & 0.1718 & 0.0033 \\
\hline$\tau_{0.55 \mu m}$ & 0.0349 & - & 0.1011 & 0.1011 & 0.1005 \\
\hline Iron & 0.0755 & 0.1011 & - & 1.000 & 0.2696 \\
\hline $\mathrm{SiC}$ & 0.0755 & 0.1011 & 1.000 & - & 0.2696 \\
\hline
\end{tabular}


Table 5.14: Continued. Correlations between modeled and collated parameters for single shell iron

\begin{tabular}{lcccccccc}
\hline & $\mathrm{T}_{\star}$ & $\mathrm{C} / \mathrm{O}$ & $\mathrm{Fe} / \mathrm{H}$ & $\mathrm{v}_{\text {exp }}$ & $\dot{M}$ & $\mathrm{~L}$ & ${ }^{12} C /{ }^{13} C$ & $\mathrm{~g} / \mathrm{d}$ \\
\hline \hline$\xi$ & 0.0062 & 0.0089 & 0.0892 & 0.0641 & 0.3216 & 0.0020 & 0.4283 & 0.1002 \\
$\tau_{0.55 \mu m}$ & 0.1136 & 0.0071 & 0.2983 & 0.0168 & 0.0660 & 0.1234 & 0.0314 & 0.1437 \\
Iron & 0.3760 & 0.0843 & 0.2326 & 0.0878 & 0.0049 & 0.0696 & 0.0955 & 0.2345 \\
$\mathrm{SiC}$ & 0.3760 & 0.0843 & 0.2326 & 0.0878 & 0.0049 & 0.0696 & 0.0955 & 0.2345 \\
\hline & & & & Log & & & & \\
\hline$\xi$ & 0.0102 & 0.0059 & 0.093 & 0.0417 & 0.0918 & 0.0098 & 0.3067 & 0.0390 \\
$\tau_{0.55 \mu m}$ & 0.1135 & 0.0066 & 0.3451 & 0.0119 & 0.2274 & 0.0352 & 0.0012 & 0.2211 \\
$\mathrm{Iron}$ & 0.3846 & 0.0638 & 0.2230 & 0.0965 & 0.0906 & 0.1594 & 0.2259 & 0.3009 \\
$\mathrm{SiC}$ & 0.3846 & 0.0638 & 0.2230 & 0.0965 & 0.0906 & 0.1594 & 0.2259 & 0.3009 \\
\hline & & & & Poly (power 2$)$ & & & \\
\hline$\xi$ & 0.3439 & 0.0661 & 0.1032 & 0.4533 & 0.4755 & 0.0020 & 0.4554 & 0.1010 \\
$\tau_{0.55 \mu m}$ & 0.1144 & 0.0073 & 0.3498 & 0.0714 & 0.1182 & 0.1970 & 0.1289 & 0.2209 \\
$\mathrm{Iron}$ & 0.3719 & 0.2662 & 0.2507 & 0.1274 & 0.1712 & 0.0851 & 0.1121 & 0.2373 \\
\hline $\mathrm{SiC}$ & 0.3719 & 0.2662 & 0.2507 & 0.1274 & 0.1712 & 0.0851 & 0.1121 & 0.2373 \\
\hline
\end{tabular}


Table 5.15: Correlations between modeled and collated parameters for single shell graphite/Fe

\begin{tabular}{|c|c|c|c|c|c|c|}
\hline & $\mathrm{T}_{\text {in }}$ & $\tau_{0.55 \mu m}$ & Graphite & $\mathrm{Fe}$ & $\mathrm{SiC}$ & $\Delta \mathrm{T}:$ Carbon $\rightarrow \mathrm{SiC}$ \\
\hline \multicolumn{7}{|c|}{ Linear } \\
\hline$\xi$ & 0.1101 & 0.0856 & 0.2853 & 0.3607 & 0.1822 & 0.1810 \\
\hline$\tau_{0.55 \mu m}$ & 0.0841 & - & 0.2907 & 0.1587 & 0.0589 & 0.0887 \\
\hline Graphite & 0.2100 & 0.2907 & - & 0.9218 & 0.0519 & 0.6185 \\
\hline Iron & 0.3503 & 0.1587 & 0.9218 & - & 0.2405 & 0.5884 \\
\hline $\mathrm{SiC}$ & 0.3900 & 0.0589 & 0.0519 & 0.2405 & - & 0.0486 \\
\hline \multicolumn{7}{|c|}{$\log$} \\
\hline$\xi$ & 0.1113 & 0.0263 & 0.3094 & 0.2101 & 0.3200 & 0.1766 \\
\hline$\tau_{0.55 \mu m}$ & 0.0794 & - & 0.2641 & - & 0.1288 & 0.0945 \\
\hline Graphite & 0.2231 & 0.2907 & - & 0.9491 & 0.0008 & 0.6323 \\
\hline Iron & 0.3627 & - & 0.9491 & - & 0.0812 & 0.6106 \\
\hline $\mathrm{SiC}$ & 0.3908 & 0.1288 & 0.0008 & 0.0812 & - & 0.0591 \\
\hline \multicolumn{7}{|c|}{ Poly (power 2) } \\
\hline$\xi$ & 0.1244 & 0.1218 & 0.3403 & 0.3888 & 0.3330 & 0.1815 \\
\hline$\tau_{0.55 \mu m}$ & 0.3384 & - & 0.3123 & 0.1602 & 0.2046 & 0.0997 \\
\hline Graphite & 0.7200 & 0.3123 & - & 0.9191 & 0.0516 & 0.6412 \\
\hline Iron & 0.7812 & 0.1602 & 0.9218 & - & 0.3398 & 0.6534 \\
\hline $\mathrm{SiC}$ & 0.3944 & 0.2046 & 0.0516 & 0.3398 & - & 0.2236 \\
\hline
\end{tabular}


Table 5.15: Continued. Correlations between modeled and collated parameters for single shell graphite/Fe

\begin{tabular}{|c|c|c|c|c|c|c|c|c|}
\hline & $\mathrm{T}_{\star}$ & $\mathrm{C} / \mathrm{O}$ & $\mathrm{Fe} / \mathrm{H}$ & $\mathrm{v}_{e x p}$ & $\dot{M}$ & $\mathrm{~L}$ & ${ }^{12} C /{ }^{13} C$ & $\mathrm{~g} / \mathrm{d}$ \\
\hline \multicolumn{9}{|c|}{ Linear } \\
\hline$\xi$ & 0.0015 & 0.1021 & 0.4747 & 0.1401 & 0.0347 & 0.0503 & 0.1037 & 0.0655 \\
\hline$\tau_{0.55 \mu m}$ & 0.2233 & 0.2078 & 0.2024 & 0.1493 & 0.0328 & 0.0744 & 0.0166 & 0.9245 \\
\hline Graphite & 0.0004 & 0.5509 & 0.6259 & 0.0015 & 0.0119 & 0.2702 & 0.1084 & \\
\hline Iron & 0.0573 & 0.3361 & 0.7378 & 0.0014 & 0.0102 & 0.2443 & 0.0332 & 0.3066 \\
\hline $\mathrm{SiC}$ & 0.3510 & 0.0865 & 0.4022 & 0.0643 & 0.0002 & 0.0103 & 0.1534 & 0.0482 \\
\hline \multicolumn{9}{|c|}{$\log$} \\
\hline$\xi$ & 0.0015 & 0.1135 & 0.3214 & 0.1625 & 0.0691 & 0.0158 & 0.1177 & 0.0198 \\
\hline$\tau_{0.55 \mu m}$ & 0.2139 & 0.1768 & 0.3468 & 0.1740 & 0.0131 & 0.0026 & 0.0006 & 0.6606 \\
\hline Graphite & 0.0021 & 0.5744 & 0.7210 & 0.0035 & 0.0286 & 0.1956 & 0.2205 & 0.2336 \\
\hline Iron & 0.0701 & 0.3708 & - & 0.0005 & 0.0255 & 0.2010 & 0.1462 & 0.1609 \\
\hline $\mathrm{SiC}$ & 0.3490 & 0.0582 & 0.2571 & 0.0675 & 0.0008 & 0.0335 & 0.1534 & 0.0135 \\
\hline \multicolumn{9}{|c|}{ Poly (power 2) } \\
\hline$\xi$ & 0.1751 & 0.1508 & 0.4941 & 0.2984 & 0.1080 & 0.0519 & 0.1890 & 0.0741 \\
\hline$\tau_{0.55 \mu m}$ & 0.2866 & 0.3922 & 0.4787 & 0.4137 & 0.1934 & 0.4406 & 0.0316 & 0.9498 \\
\hline Graphite & 0.4831 & 0.7888 & 0.1041 & 0.0164 & 0.3366 & 0.4917 & 0.5737 & 0.4857 \\
\hline Iron & 0.4857 & 0.4548 & 0.6376 & 0.0599 & 0.0127 & 0.2735 & 0.6354 & 0.3091 \\
\hline $\mathrm{SiC}$ & 0.3523 & 0.4567 & 0.4219 & 0.0888 & 0.0014 & 0.0538 & 0.4861 & 0.4390 \\
\hline
\end{tabular}


Table 5.16: Correlations between modeled and collated parameters for single shell

\begin{tabular}{|c|c|c|c|c|c|c|}
\hline & $\mathrm{T}_{\text {in }}$ & $\tau_{0.55 \mu m}$ & $\mathrm{AmC}$ & $\mathrm{Fe}$ & $\mathrm{SiC}$ & $\Delta \mathrm{T}:$ Carbon $\rightarrow \mathrm{SiC}$ \\
\hline \multicolumn{7}{|c|}{ Linear } \\
\hline$\xi$ & 0.1101 & 0.0856 & 0.3244 & 0.3607 & 0.0334 & 0.1810 \\
\hline$\tau_{0.55 \mu m}$ & 0.0841 & - & 0.2430 & 0.1587 & 0.1582 & 0.0887 \\
\hline $\mathrm{AmC}$ & 0.0783 & 0.2430 & - & 0.8965 & 0.0314 & 0.3529 \\
\hline Iron & 0.4224 & 0.1587 & 0.8965 & - & 0.2347 & 0.3819 \\
\hline $\mathrm{SiC}$ & 0.4238 & 0.1288 & 0.0008 & 0.0812 & - & 0.0860 \\
\hline \multicolumn{7}{|c|}{$\log$} \\
\hline$\xi$ & 0.1113 & 0.0263 & 0.3420 & 0.2101 & 0.0014 & 0.1766 \\
\hline$\tau_{0.55 \mu m}$ & 0.2139 & 0.0794 & 0.2712 & 0.2974 & 0.2794 & 0.0945 \\
\hline $\mathrm{AmC}$ & 0.0836 & 0.2974 & - & 0.9030 & 0.0024 & 0.3690 \\
\hline Iron & 0.4330 & 0.2794 & 0.9030 & - & 0.1160 & 0.4318 \\
\hline $\mathrm{SiC}$ & 0.4210 & 0.2046 & 0.0516 & 0.3398 & - & 0.0985 \\
\hline \multicolumn{7}{|c|}{ Poly (power 2) } \\
\hline$\xi$ & 0.1244 & 0.1218 & 0.3793 & 0.3888 & 0.0464 & 0.1815 \\
\hline$\tau_{0.55 \mu m}$ & 0.3384 & - & 0.3216 & 0.4197 & 0.2230 & 0.0997 \\
\hline $\mathrm{AmC}$ & 0.4518 & 0.3216 & - & 0.9029 & 0.0318 & 0.6631 \\
\hline Iron & 0.6376 & 0.2230 & 0.9491 & - & 0.2350 & 0.6534 \\
\hline $\mathrm{SiC}$ & 0.4384 & 0.2230 & 0.0318 & 0.2350 & - & 0.1494 \\
\hline
\end{tabular}


Table 5.16: Continued. Correlations between modeled and collated parameters for single shell $\mathrm{AmC} / \mathrm{Fe}$

\begin{tabular}{|c|c|c|c|c|c|c|c|c|}
\hline & $\mathrm{T}_{\star}$ & $\mathrm{C} / \mathrm{O}$ & $\mathrm{Fe} / \mathrm{H}$ & $\mathrm{v}_{e x p}$ & $\dot{M}$ & $\mathrm{~L}$ & ${ }^{12} \mathrm{C} /{ }^{13} \mathrm{C}$ & $\mathrm{g} / \mathrm{d}$ \\
\hline \multicolumn{9}{|c|}{ Linear } \\
\hline$\xi$ & 0.0005 & 0.1021 & 0.4747 & 0.1404 & 0.0347 & 0.0503 & 0.1037 & 0.0655 \\
\hline$\tau_{0.55 \mu m}$ & 0.2233 & 0.2078 & 0.2024 & 0.1493 & 0.0328 & 0.0744 & 0.0166 & 0.9245 \\
\hline $\mathrm{AmC}$ & 0.1215 & 0.1093 & 0.8369 & 0.0018 & 0.0051 & 0.3782 & 0.0237 & 0.1689 \\
\hline Iron & 0.3173 & 0.3355 & 0.8844 & 0.0002 & 0.0011 & 0.2575 & 0.0974 & 0.0420 \\
\hline $\mathrm{SiC}$ & 0.4238 & 0.1148 & 0.1515 & 0.0055 & 0.0868 & 0.0144 & 0.2856 & 0.0860 \\
\hline \multicolumn{9}{|c|}{$\log$} \\
\hline$\xi$ & 0.0015 & 0.1135 & 0.3214 & 0.1625 & 0.0691 & 0.0158 & 0.1177 & 0.0198 \\
\hline$\tau_{0.55 \mu m}$ & 0.2139 & 0.3468 & 0.1740 & 0.1740 & 0.0131 & 0.0026 & 0.0006 & 0.6606 \\
\hline $\mathrm{AmC}$ & 0.1348 & 0.1290 & 0.8356 & 0.0020 & 0.0037 & 0.3138 & 0.1296 & 0.0672 \\
\hline Iron & 0.3316 & 0.0534 & - & 0.0000 & 0.0087 & 0.2192 & 0.0303 & 0.175 \\
\hline $\mathrm{SiC}$ & 0.4210 & 0.0728 & 0.0296 & 0.0096 & 0.2034 & 0.0083 & 0.1049 & 0.0985 \\
\hline \multicolumn{9}{|c|}{ Poly (power 2) } \\
\hline$\xi$ & 0.1751 & 0.1508 & 0.4941 & 0.2984 & 0.1080 & 0.0519 & 0.1890 & 0.0741 \\
\hline$\tau_{0.55 \mu m}$ & 0.2866 & 0.3922 & 0.4787 & 0.4137 & 0.1934 & 0.4406 & 0.0316 & 0.9029 \\
\hline $\mathrm{AmC}$ & 0.3511 & 0.2125 & 0.8475 & 0.0055 & 0.0351 & 0.4115 & 0.4396 & 0.1794 \\
\hline Iron & 0.4322 & 0.3385 & 0.8904 & 0.0599 & 0.0127 & 0.2735 & 0.6354 & 0.3091 \\
\hline $\mathrm{SiC}$ & 0.4384 & 0.7803 & 0.1981 & 0.1692 & 0.2200 & 0.0319 & 0.4965 & 0.1494 \\
\hline
\end{tabular}




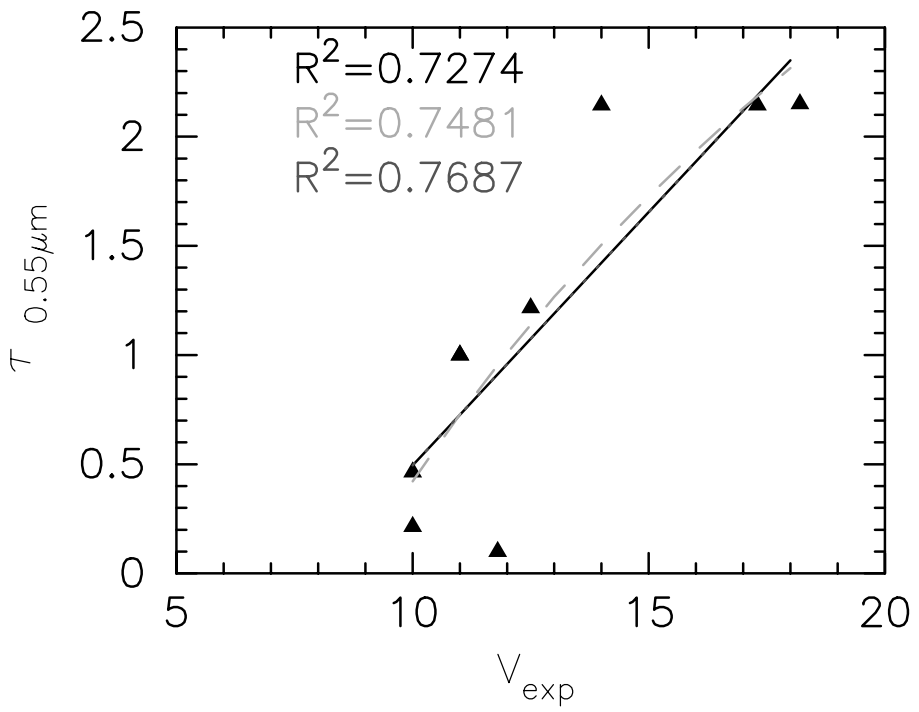

Figure 5.10: The correlations between the optical depth and the expasion velocity for AmC single shell models.

Table 5.17: Correlations Amongst Collated 2-Shell Parameters

\begin{tabular}{lccccc} 
& $\mathrm{T}_{\star}$ & $\mathrm{T}_{\text {in }}$ & $\mathrm{C} / \mathrm{O}$ & $\mathrm{Fe} / \mathrm{H}$ & $\mathrm{v}_{\text {exp }}$ \\
\hline \hline \multicolumn{6}{c}{ Linear } \\
\hline $\mathrm{T}_{2^{\text {nd dust }}}$ & 0.0121 & 0.3740 & 0.0018 & 0.0000 & 0.1394 \\
\hline \multicolumn{5}{c}{$\mathrm{Log}$} \\
\hline $\mathrm{T}_{2^{\text {nd dust }}}$ & 0.0121 & 0.3728 & 0.0323 & 0.0000 & 0.0952 \\
\hline \multicolumn{5}{c}{ Poly (power 2) } \\
\hline $\mathrm{T}_{2^{\text {nd dust }}}$ & 0.0133 & 0.3768 & 0.0800 & 0.0230 & 0.7740 \\
\hline
\end{tabular}




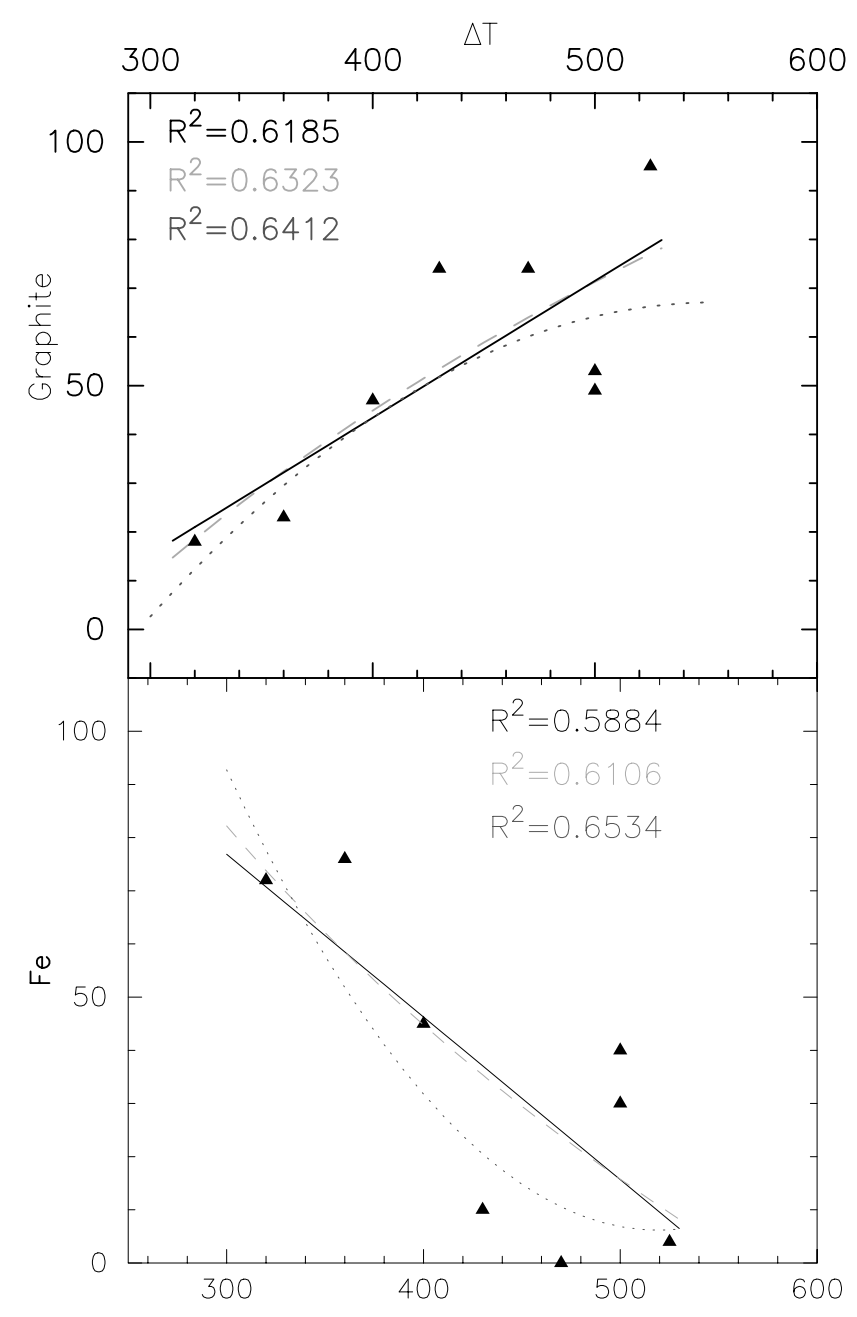

Figure 5.11: The correlations between the amount of graphite grains and change of temperature between the formation of graphite to $\mathrm{SiC}$ grains and the amount of iron grains and change of temperature between the formation of graphite to $\mathrm{SiC}$ grains for graphite/Fe single shell models. 


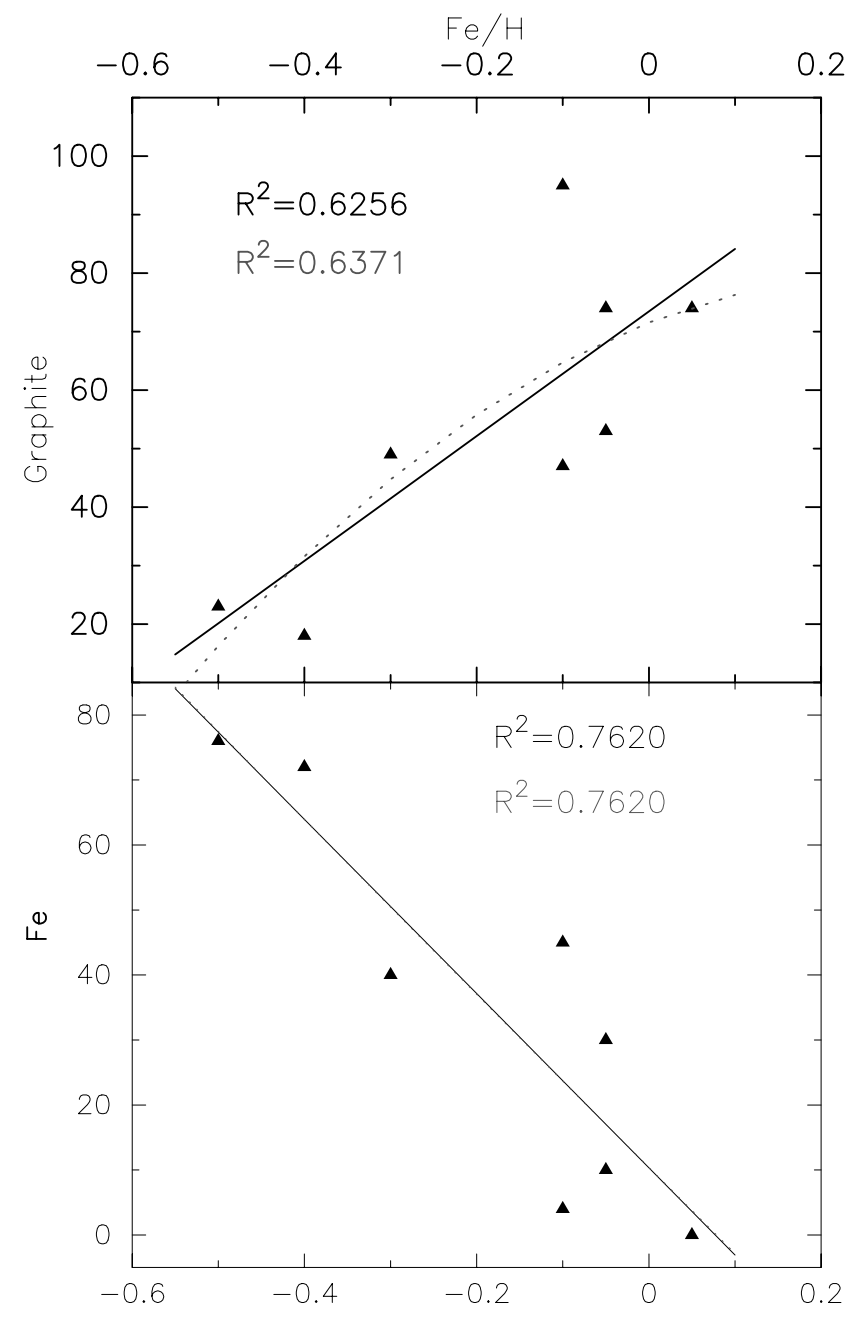

Figure 5.12: The correlations between the amount of graphite grains and $\mathrm{Fe} / \mathrm{H}$ ratio and the amount of iron grains and $\mathrm{Fe} / \mathrm{H}$ for graphite/Fe single shell models. 


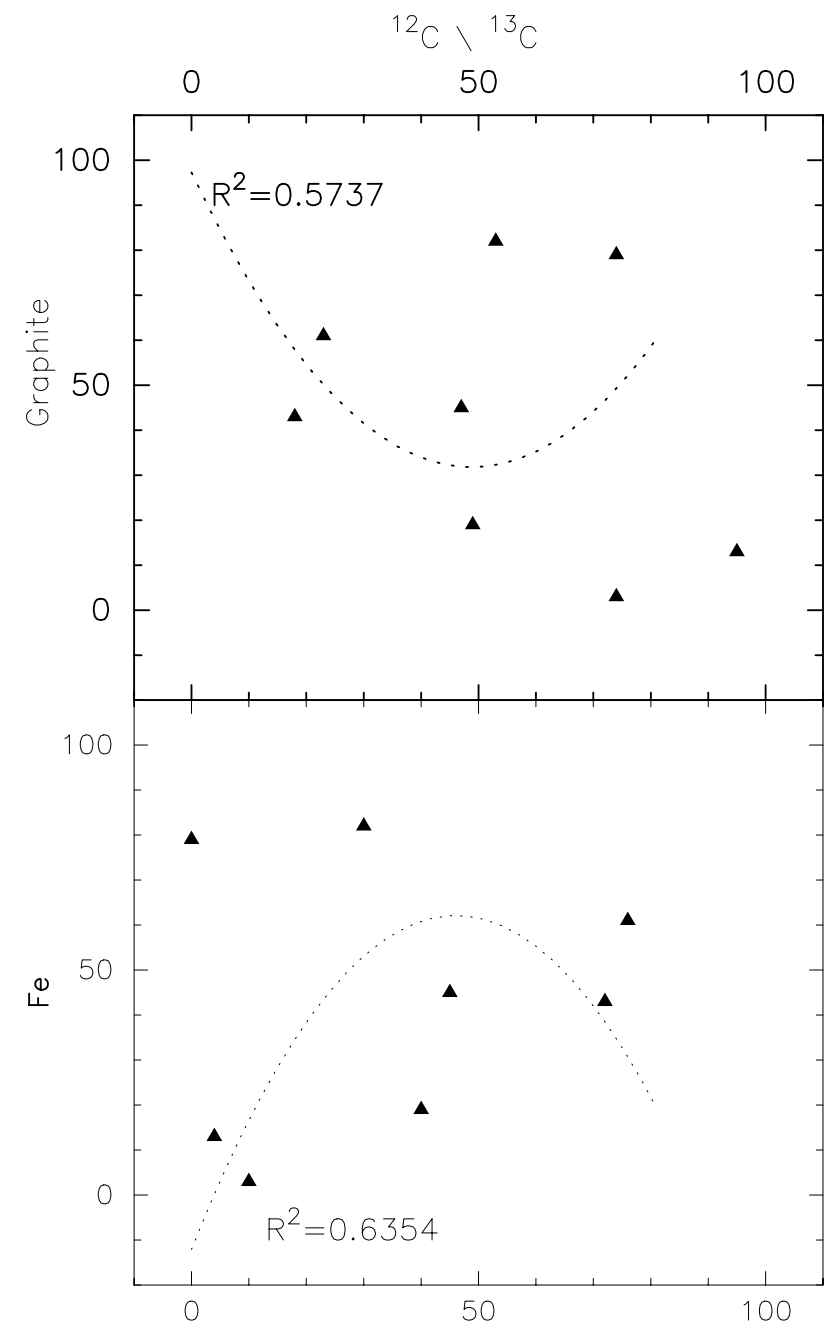

Figure 5.13: The correlations between the amount of graphite grains and ${ }^{12} \mathrm{C} /{ }^{13} \mathrm{C}$ ratio and the amount of iron grains and ${ }^{12} \mathrm{C} /{ }^{13} \mathrm{C}$ ratio for graphite/Fe single shell models. 


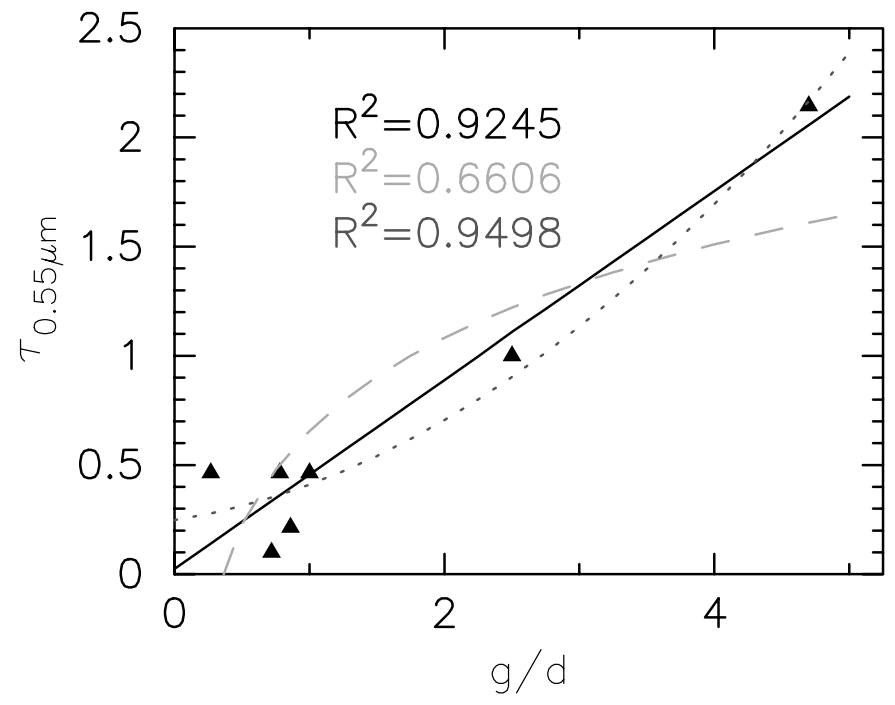

Figure 5.14: The correlations between the optical depth and gas-to-dust ratio for graphite/Fe single shell models.

Table 5.17: Continued. Correlations Amongst Collated 2-Shell Parameters

\begin{tabular}{cccccc} 
& $\dot{M}$ & $\mathrm{~L}$ & ${ }^{12} C /{ }^{13} C$ & $\mathrm{~g} / \mathrm{d}$ & $\Delta \mathrm{T}:$ Carbon $\rightarrow \mathrm{SiC}$ \\
\hline \hline \multicolumn{5}{c}{ Linear } \\
\hline $\mathrm{T}_{2^{\text {nd dust }}}$ & 0.3136 & 0.0397 & 0.0015 & 0.2856 & 0.0482 \\
\hline \multicolumn{5}{c}{$\mathrm{Log}$} \\
\hline $\mathrm{T}_{2^{\text {nd dust }}}$ & 0.4410 & 0.0537 & 0.4748 & 0.4676 & 0.0639 \\
\hline & \multicolumn{5}{c}{ Poly (power 2) } \\
\hline $\mathrm{T}_{2^{\text {nd dust }}}$ & 0.4088 & 0.0675 & 0.1012 & 0.4676 & 0.3558 \\
\hline
\end{tabular}




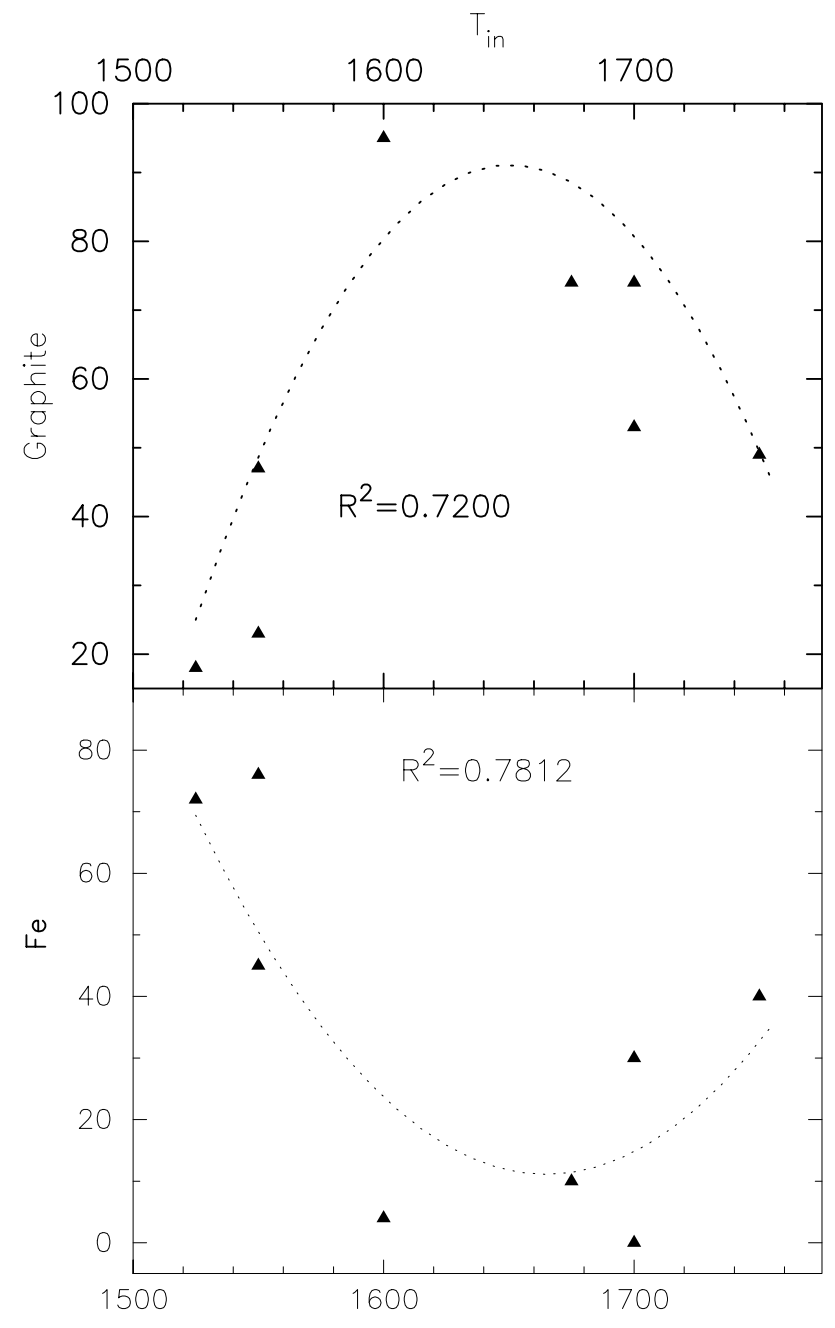

Figure 5.15: The correlations between the amount of graphite grains and temperture of the inner dust shell and the amount of iron grains and temparture of the inner dust shell for graphite/Fe single shell models. 


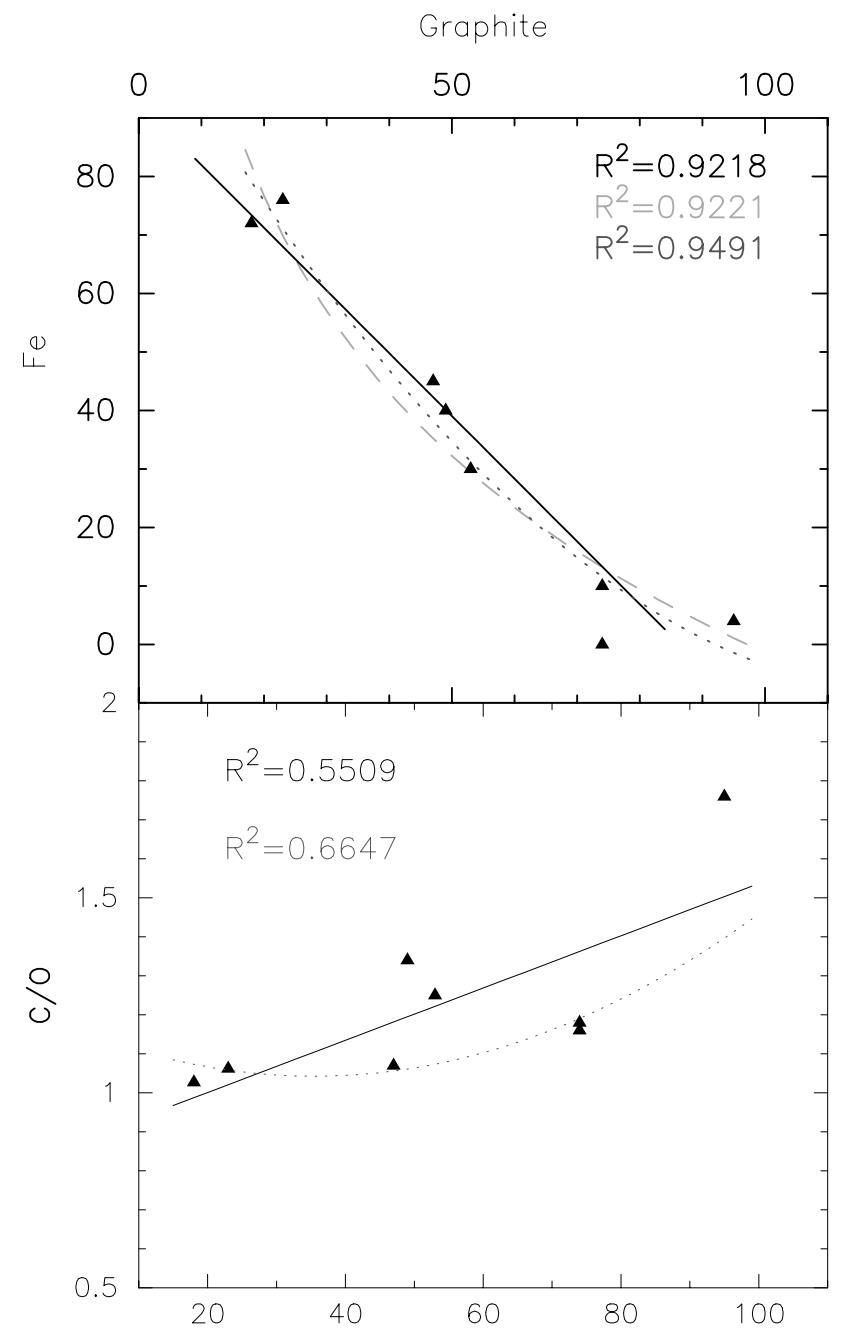

Figure 5.16: The correlations between the amount of iron grains and graphite grains and the $\mathrm{C} / \mathrm{O}$ ratio and the amount of graphite grains for graphite/Fe single shell models. 


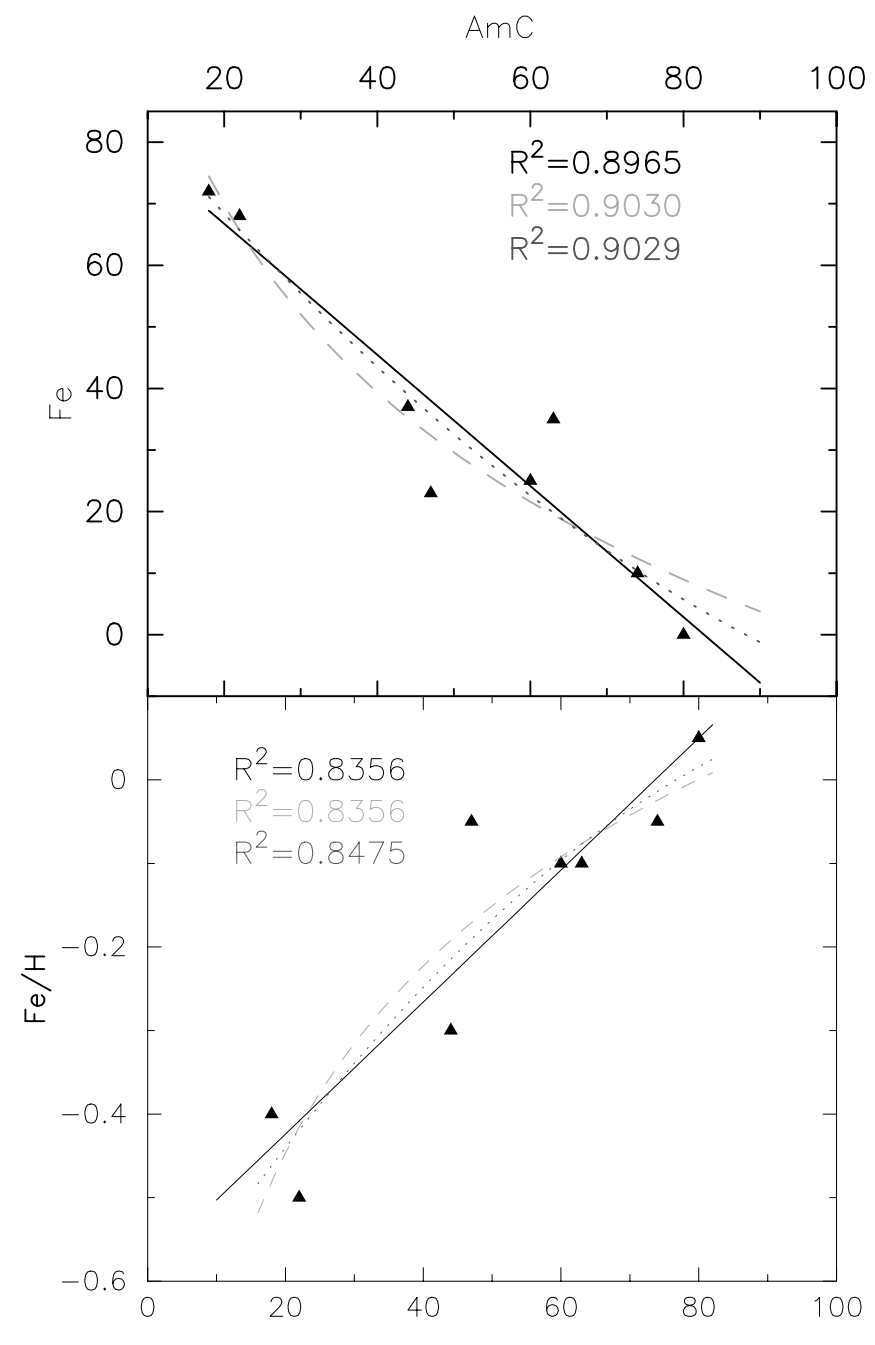

Figure 5.17: The correlations between the amount of iron grains and AmC grains and the $\mathrm{Fe} / \mathrm{H}$ ratio and the amount of $\mathrm{AmC}$ grains for $\mathrm{AmC} / \mathrm{Fe}$ single shell models. 


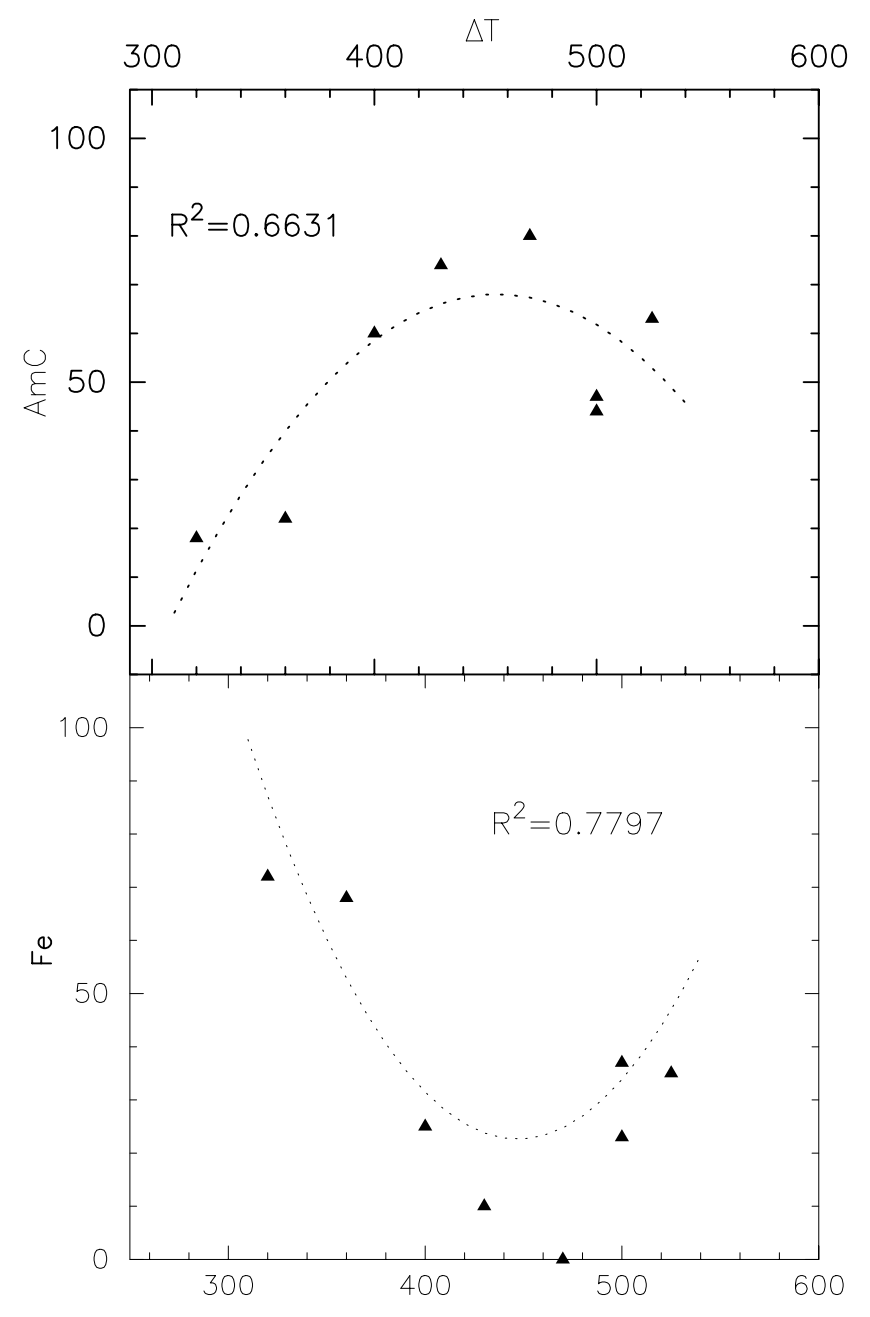

Figure 5.18: The correlations between the amount of $\mathrm{AmC}$ grains and change of temperature between the formation of graphite to $\mathrm{SiC}$ grains and the amount of iron grains and change of temperature between the formation of graphite to $\mathrm{SiC}$ grains for AmC/Fe single shell models. 


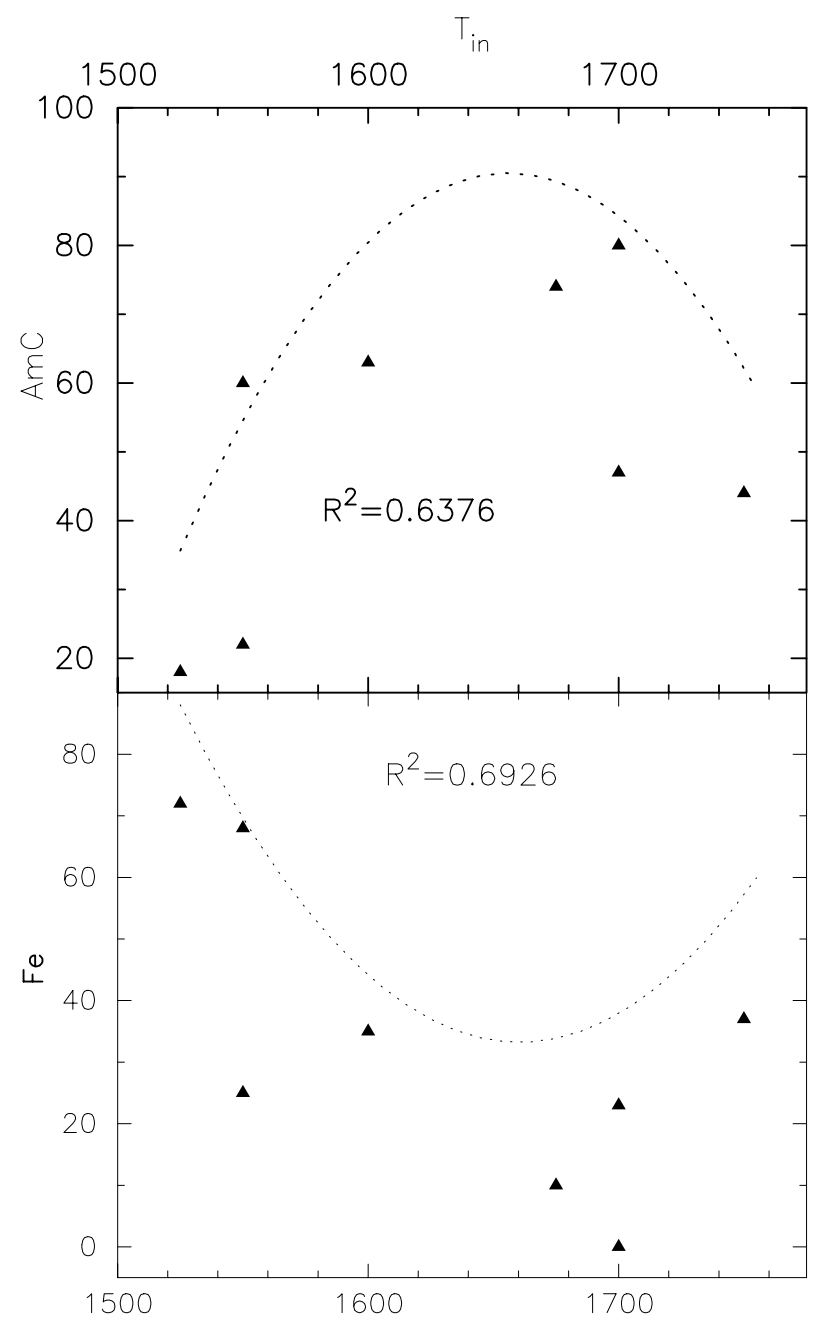

Figure 5.19: The correlations between the amount of $\mathrm{AmC}$ grains and the temperature of the inner dust shell and the amount of iron grains and the temperature of the inner dust shell for AmC/Fe single shell models. 


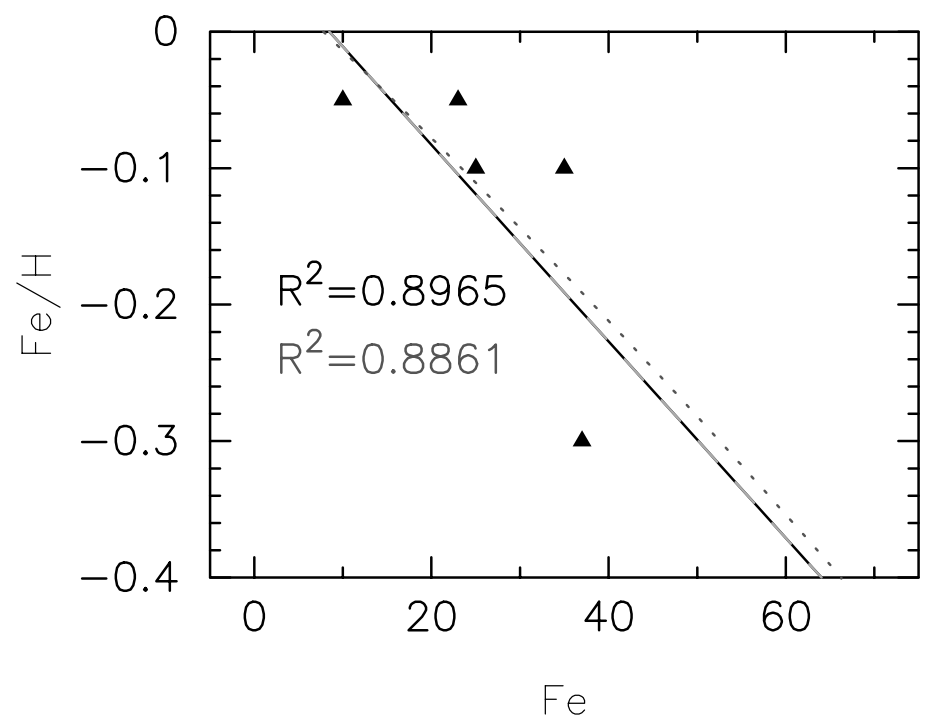

Figure 5.20: The correlations between the $\mathrm{Fe} / \mathrm{H}$ ratio and the amount of iron in AmC/Fe single shell models.

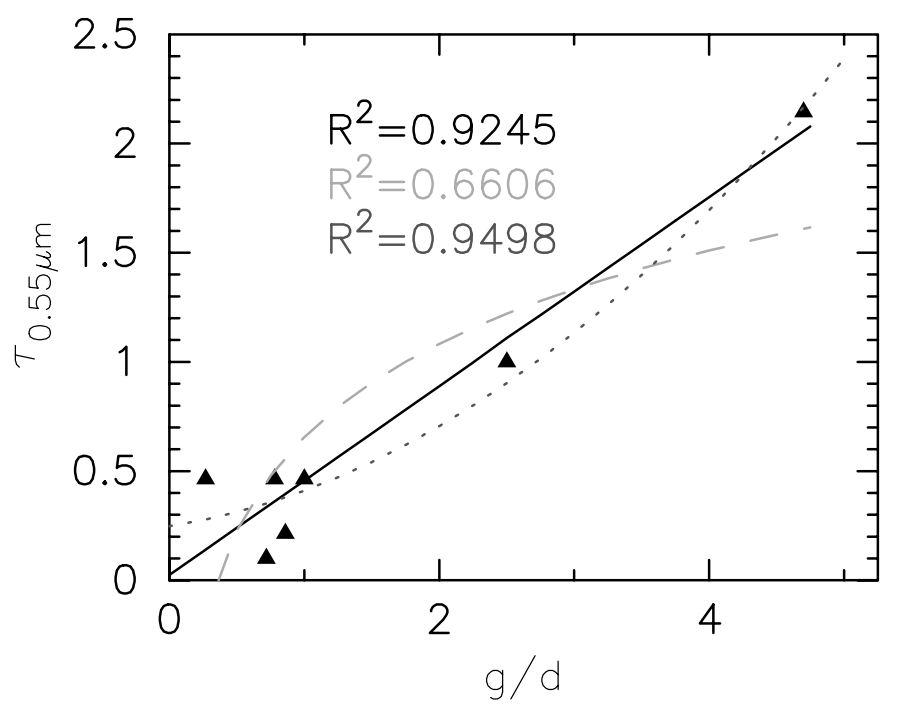

Figure 5.21: The correlations between the optical depth and gas-to-dust ratio for AmC/Fe single shell models. 
Table 5.18: Correlations between modeled and collated parameters for 2 shell graphite

\begin{tabular}{lcccccc} 
& $\mathrm{T}_{\text {in }}$ & $\mathrm{T}_{\text {out }}$ & $\tau_{0.55 \mu \text { mout }}$ & Graphite & $\mathrm{SiC}$ & $\Delta \mathrm{T}$ : Carbon $\rightarrow \mathrm{SiC}$ \\
\hline \hline$\tau_{0.55 \mu \text { min }}$ & 0.0052 & 0.0054 & 0.0042 & $\mathbf{0 . 6 3 1 0}$ & $\mathbf{0 . 6 3 1 0}$ & 0.0609 \\
$\tau_{0.55 \mu \text { mout }}$ & 0.0620 & 0.0043 & - & 0.3696 & 0.3696 & 0.0137 \\
Graphite & 0.0614 & 0.0003 & 0.3696 & - & $\mathbf{1 . 0 0 0}$ & 0.0801 \\
SiC & 0.0614 & 0.0003 & 0.3696 & $\mathbf{1 . 0 0 0}$ & - & 0.0801 \\
\hline & & & \multicolumn{7}{c}{ Logarythmic } & \\
\hline$\tau_{0.55 \mu \text { min }}$ & 0.0038 & 0.0052 & 0.0442 & $\mathbf{0 . 7 8 5 4}$ & $\mathbf{0 . 7 8 5 4}$ & 0.0632 \\
$\tau_{0.55 \mu \text { mout }}$ & 0.0566 & 0.0049 & - & 0.2703 & 0.4271 & 0.0149 \\
Graphite & 0.1572 & 0.0001 & 0.0631 & - & $\mathbf{1 . 0 0 0}$ & 0.0843 \\
SiC & 0.1572 & 0.0001 & 0.0631 & $\mathbf{1 . 0 0 0}$ & - & 0.0843 \\
\hline & & & $2 \mathrm{nd}$ order polynomial & \\
\hline$\tau_{0.55 \mu \text { min }}$ & 0.3679 & 0.0358 & 0.0442 & $\mathbf{0 . 5 2 4 3}$ & $\mathbf{0 . 5 2 4 3}$ & 0.0613 \\
$\tau_{0.55 \mu \text { mout }}$ & 0.4471 & 0.2500 & - & 0.4154 & 0.4154 & 0.0247 \\
Graphite & 0.4350 & 0.2658 & 0.4154 & - & $\mathbf{1 . 0 0 0}$ & 0.0861 \\
SiC & 0.4350 & 0.2658 & 0.4154 & $\mathbf{1 . 0 0 0}$ & - & 0.0861 \\
\hline
\end{tabular}


Table 5.18: Continued. Correlations between modeled and collated parameters for 2 shell graphite

\begin{tabular}{|c|c|c|c|c|c|c|c|c|}
\hline & $\mathrm{T}_{\star}$ & $\mathrm{C} / \mathrm{O}$ & $\mathrm{Fe} / \mathrm{H}$ & $\mathrm{v}_{e x p}$ & $\dot{M}$ & $\mathrm{~L}$ & ${ }^{12} \mathrm{C} /{ }^{13} \mathrm{C}$ & $g / d$ \\
\hline \multicolumn{9}{|c|}{ Linear } \\
\hline$\tau_{0.55 \mu m i n}$ & 0.2225 & 0.2254 & 0.0466 & 0.2877 & 0.0633 & 0.0259 & 0.0388 & 0.0726 \\
\hline$\tau_{0.55 \mu \text { mout }}$ & 0.1137 & 0.0081 & 0.2835 & 0.0807 & 0.2031 & 0.0044 & 0.0627 & 0.4495 \\
\hline Graphite & 0.0614 & 0.1479 & 0.0404 & 0.0111 & 0.1753 & 0.0614 & 0.0138 & 0.0662 \\
\hline $\mathrm{SiC}$ & 0.0614 & 0.1479 & 0.0404 & 0.0111 & 0.1753 & 0.0326 & 0.0138 & 0.0662 \\
\hline \multicolumn{9}{|c|}{ Logarythmic } \\
\hline$\tau_{0.55 \mu m i n}$ & 0.2116 & 0.1908 & 0.0682 & 0.3215 & 0.2894 & 0.0017 & 0.0444 & 0.0885 \\
\hline$\tau_{0.55 \mu m o u t}$ & 0.1075 & 0.0040 & 0.2835 & 0.0865 & 0.0574 & 0.0242 & 0.0383 & 0.4353 \\
\hline Graphite & 0.0544 & 0.1314 & 0.0004 & 0.0109 & 0.1728 & 0.0026 & 0.0081 & 0.0550 \\
\hline $\mathrm{SiC}$ & 0.0544 & 0.1314 & 0.0004 & 0.0109 & 0.1728 & 0.0026 & 0.0081 & 0.0550 \\
\hline \multicolumn{9}{|c|}{ 2nd order polynomial } \\
\hline$\tau_{0.55 \mu m i n}$ & 0.3157 & 0.4279 & 0.0715 & 0.4865 & 0.1897 & 0.3998 & 0.0950 & 0.2142 \\
\hline$\tau_{0.55 \mu m o u t}$ & 0.1639 & 0.1197 & 0.2835 & 0.0866 & 0.3190 & 0.0892 & 0.0629 & 0.4831 \\
\hline Graphite & 0.2079 & 0.2219 & 0.3964 & 0.0216 & 0.2259 & 0.2435 & 0.0147 & 0.0837 \\
\hline $\mathrm{SiC}$ & 0.2079 & 0.2219 & 0.3964 & 0.0216 & 0.2259 & 0.2435 & 0.0147 & 0.0837 \\
\hline
\end{tabular}


Table 5.19: Correlations between modeled and collated parameters for 2 shell AmC

\begin{tabular}{|c|c|c|c|c|c|c|}
\hline & $\mathrm{T}_{\text {in }}$ & $\mathrm{T}_{\text {out }}$ & $\tau_{0.55 \mu m o u t}$ & $\mathrm{AmC}$ & $\mathrm{SiC}$ & $\Delta \mathrm{T}:$ Carbon $\rightarrow \mathrm{SiC}$ \\
\hline \multicolumn{7}{|c|}{ Linear } \\
\hline$\tau_{0.55 \mu m i n}$ & 0.0052 & 0.0054 & 0.0042 & 0.3696 & 0.3696 & 0.0609 \\
\hline$\tau_{0.55 \mu m o u t}$ & 0.0620 & 0.0043 & - & 0.2930 & 0.2930 & 0.0137 \\
\hline $\mathrm{AmC}$ & 0.0090 & 0.0126 & 0.2930 & - & 1.000 & 0.2975 \\
\hline $\mathrm{SiC}$ & 0.0000 & 0.0003 & 0.3696 & 1.000 & - & 0.0801 \\
\hline \multicolumn{7}{|c|}{$\log$} \\
\hline$\tau_{0.55 \mu m i n}$ & 0.0038 & 0.0052 & 0.0442 & 0.4514 & 0.4514 & 0.0632 \\
\hline$\tau_{o 0.55 \mu m o u t}$ & 0.0566 & 0.0049 & - & 0.1935 & 0.1935 & 0.0149 \\
\hline $\mathrm{AmC}$ & 0.0121 & 0.0122 & 0.1935 & - & 1.000 & 0.2995 \\
\hline $\mathrm{SiC}$ & 0.0121 & 0.0122 & 0.1935 & 1.000 & - & 0.2995 \\
\hline \multicolumn{7}{|c|}{ 2nd order Polynomial } \\
\hline$\tau_{0.55 \mu m i n}$ & 0.3679 & 0.0358 & 0.0442 & 0.4468 & 0.4468 & 0.0613 \\
\hline$\tau_{0.55 \mu \text { mout }}$ & 0.4471 & 0.2500 & - & 0.3173 & 0.3173 & 0.0247 \\
\hline $\mathrm{AmC}$ & 0.7257 & 0.0810 & 0.3173 & - & 1.000 & 0.2975 \\
\hline $\mathrm{SiC}$ & 0.7257 & 0.0810 & 0.3173 & 1.000 & - & 0.2975 \\
\hline
\end{tabular}


Table 5.19: Conintued. Correlations between modeled and collated parameters for 2 shell AmC

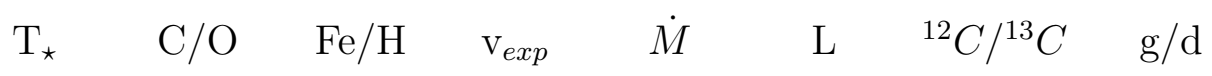

\begin{tabular}{|c|c|c|c|c|c|c|c|c|}
\hline \multicolumn{9}{|c|}{ Linear } \\
\hline$\tau_{0.55 \mu m i n}$ & 0.2225 & 0.2254 & 0.0466 & 0.2877 & 0.0633 & 0.0259 & 0.0388 & 0.0726 \\
\hline$\tau_{0.55 \mu \text { mout }}$ & 0.1137 & 0.0081 & 0.2835 & 0.0807 & 0.2031 & 0.0044 & 0.0627 & 0.4495 \\
\hline $\mathrm{AmC}$ & 0.1082 & 0.4000 & 0.4700 & 0.0007 & 0.0076 & 0.1620 & 0.2473 & 0.0019 \\
\hline $\mathrm{SiC}$ & 0.0614 & 0.1479 & 0.0404 & 0.0111 & 0.1753 & 0.0326 & 0.0138 & 0.0662 \\
\hline \multicolumn{9}{|c|}{$\log$} \\
\hline$\tau_{0.55 \mu m i n}$ & 0.2116 & 0.1908 & 0.0682 & 0.3215 & 0.2894 & 0.0017 & 0.0444 & 0.0885 \\
\hline$\tau_{o 0.55 \mu m o u t}$ & 0.1075 & 0.0040 & 0.2835 & 0.0865 & 0.0574 & 0.0242 & 0.0383 & 0.4353 \\
\hline $\mathrm{AmC}$ & 0.0946 & 0.4472 & 0.0013 & 0.0048 & 0.0965 & 0.1329 & 0.0000 & 0.0375 \\
\hline $\mathrm{SiC}$ & 0.0946 & 0.4472 & 0.0013 & 0.0048 & 0.0965 & 0.1329 & 0.0000 & 0.0375 \\
\hline \multicolumn{9}{|c|}{ 2nd order Polynomial } \\
\hline$\tau_{0.55 \mu m i n}$ & 0.3157 & 0.4279 & 0.0715 & 0.4865 & 0.1897 & 0.3998 & 0.0950 & 0.2142 \\
\hline$\tau_{0.55 \mu m o u t}$ & 0.1639 & 0.1197 & 0.2835 & 0.0866 & 0.3190 & 0.0892 & 0.0629 & 0.4831 \\
\hline $\mathrm{AmC}$ & 0.4319 & 0.5123 & 0.0120 & 0.0863 & 0.1940 & 0.3335 & 0.0020 & 0.1569 \\
\hline $\mathrm{SiC}$ & 0.4319 & 0.5123 & 0.0120 & 0.0863 & 0.1940 & 0.3335 & 0.0020 & $0.1569]$ \\
\hline
\end{tabular}


Table 5.20: Correlations betweens the $\mathrm{SiC}$ Abundances for models with different modeled continuum dust species

\begin{tabular}{lcc}
\hline Parameter 1 & Parameter 2 & $\mathrm{R}^{2}$ \\
\hline \hline Graphite & Linear \\
Graphite & Iron & $\mathbf{0 . 5 7 1 0}$ \\
Graphite & Graphite/Iron & $\mathbf{0 . 9 5 0 1}$ \\
Graphite & AmC/Fe & $\mathbf{0 . 6 3 2 2}$ \\
AmC & Iron & $\mathbf{0 . 8 3 3 4}$ \\
AmC & Graphite/Iron & $\mathbf{0 . 5 3 0 6}$ \\
AmC & AmC/Iron & $\mathbf{0 . 6 7 0 5}$ \\
Iron & Graphite/Iron & 0.2423 \\
Iron & AmC/Iron & 0.3791 \\
AmC/Iron & Graphite/Iron & $\mathbf{0 . 5 2 2 4}$ \\
\hline
\end{tabular}


Table 5.20: Continued. Correlations between the SiC Abundances for models with different modeled continuum dust species

\begin{tabular}{lcc}
\hline Parameter 1 & Parameter 2 & $\mathrm{R}^{2}$ \\
\hline Lraphite & AmC & $\mathbf{0 . 6 3 9 2}$ \\
Graphite & Iron & 0.2800 \\
Graphite & Graphite/Iron & $\mathbf{0 . 7 0 8 0}$ \\
Graphite & AmC/Fe & $\mathbf{0 . 6 8 1 4}$ \\
AmC & Iron & $\mathbf{0 . 5 3 6 3}$ \\
AmC & Graphite/Iron & $\mathbf{0 . 5 2 3 7}$ \\
AmC & AmC/Iron & $\mathbf{0 . 7 0 4 9}$ \\
Iron & Graphite/Iron & $\mathbf{0 . 3 9 9 6}$ \\
Iron & AmC/Iron & $\mathbf{0 . 6 5 5 8}$ \\
AmC/Iron & Graphite/Iron & $\mathbf{0 . 5 7 2 6}$ \\
\hline
\end{tabular}


Table 5.20: Continued. Correlations betweens the SiC Abundances for models with different modeled continuum dust species

\begin{tabular}{lcc}
\hline Parameter 1 & Parameter 2 & $\mathrm{R}^{2}$ \\
\hline Poly (power 2) \\
\hline Graphite & AmC & $\mathbf{0 . 7 5 3 8}$ \\
Graphite & Iron & $\mathbf{0 . 5 0 8 0}$ \\
Graphite & Graphite/Iron & $\mathbf{0 . 9 5 0 8}$ \\
Graphite & AmC/Fe & $\mathbf{0 . 7 3 0 0}$ \\
AmC & Iron & $\mathbf{0 . 9 1 4 8}$ \\
AmC & Graphite/Iron & $\mathbf{0 . 6 1 9 1}$ \\
AmC & AmC/Iron & $\mathbf{0 . 7 6 7 0}$ \\
Iron & Graphite/Iron & $\mathbf{0 . 3 9 6 6}$ \\
Iron & AmC/Iron & $\mathbf{0 . 8 2 0 0}$ \\
AmC/Iron & Graphite/Iron & $\mathbf{0 . 6 2 5 2}$ \\
\hline
\end{tabular}




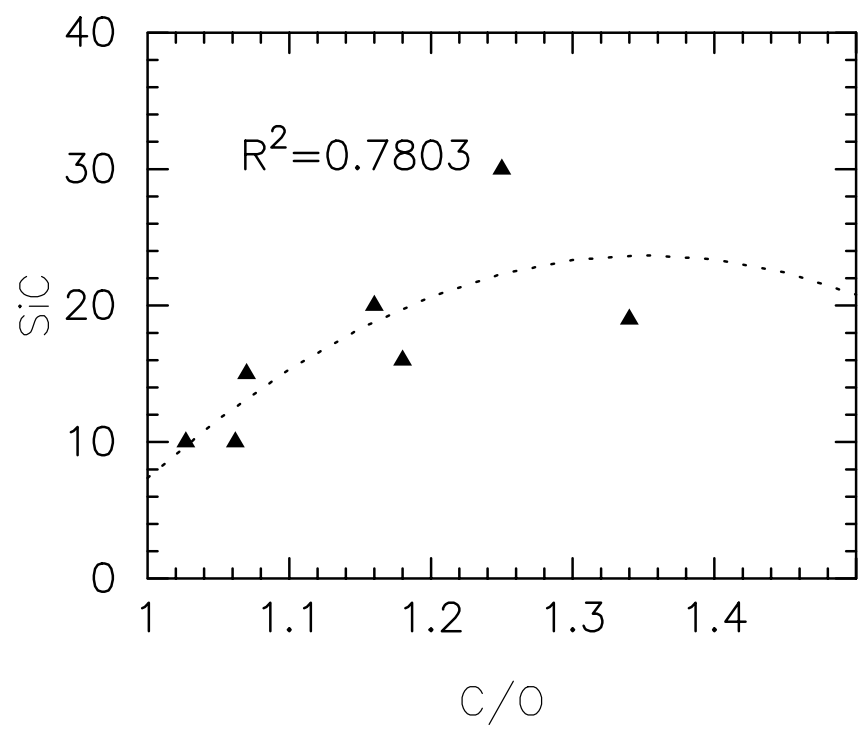

Figure 5.22: The correlations between the abundances $\mathrm{SiC}$ grains and $\mathrm{C} / \mathrm{O}$ ratio in AmC/Fe single shell models.

(1) iron grains and graphite or AmC grains (see Tables 4.15 and 5.16), (2) $\tau_{0.55 \mu m}$ and gas-to-dust ratio (see Tables 4.15 and 5.16), (3) graphite or AmC grains and $\mathrm{Fe} / \mathrm{H}$ (see Tables 4.15 and 5.16), and (4) iron grains and Fe/H (see Tables 4.15 and 5.16). While signification correlations exist for iron grains and graphite or AmC grains and graphite, $\mathrm{AmC}$ and iron grains with $\mathrm{Fe} / \mathrm{H}$, these are excepted since these parameters depend on each other since the amount of iron grains is derived from the $\mathrm{Fe} / \mathrm{H}$ and $\mathrm{C} / \mathrm{H}$ data. With the abundance of $\mathrm{SiC}$ grains between the different models there are correlations between all the models except for when one of the modeled continuum was dominated by iron. We will discuss these correlations further in, $\S 5.2 .3$. 


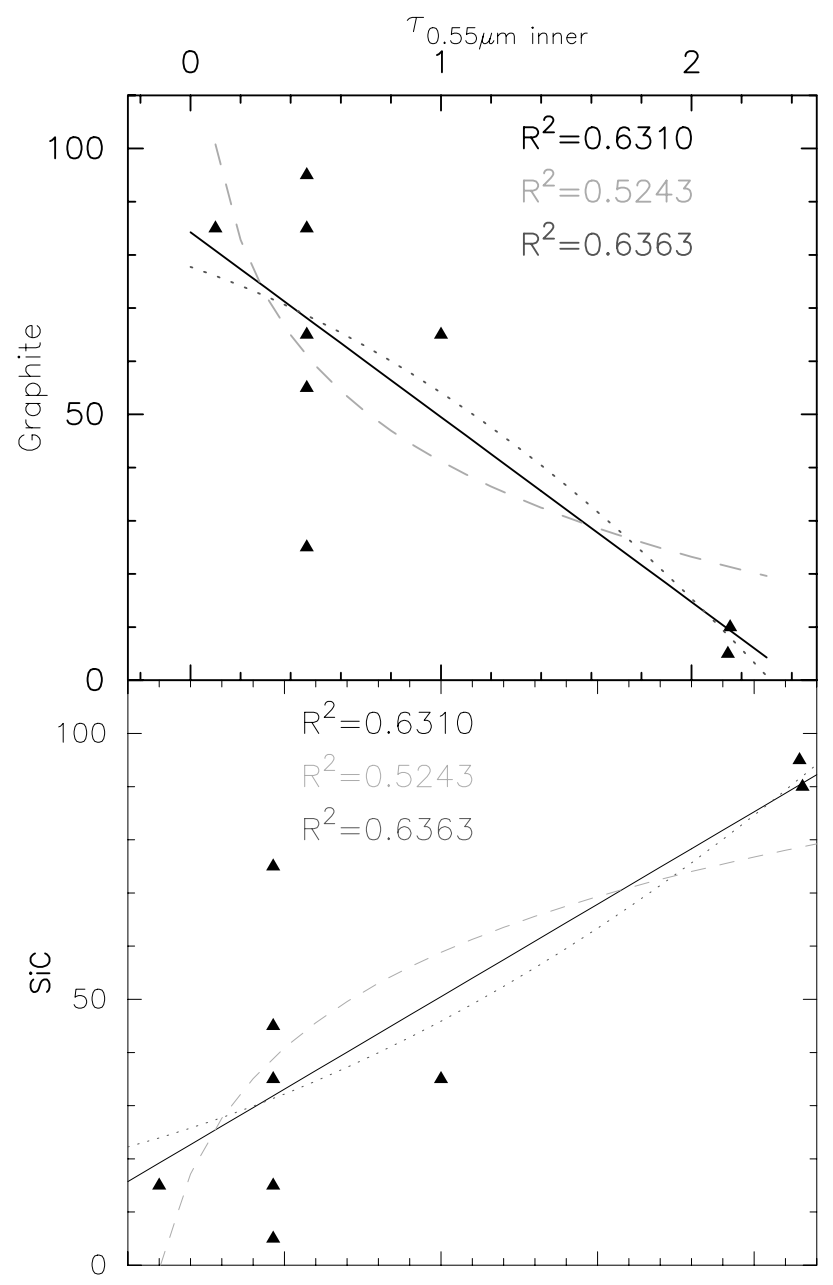

Figure 5.23: The correlations between the amount of graphite grains and the temperature of the inner dust shell and the amount of $\mathrm{SiC}$ grains and the temperature of the inner dust shell for graphite 2-shells models. 


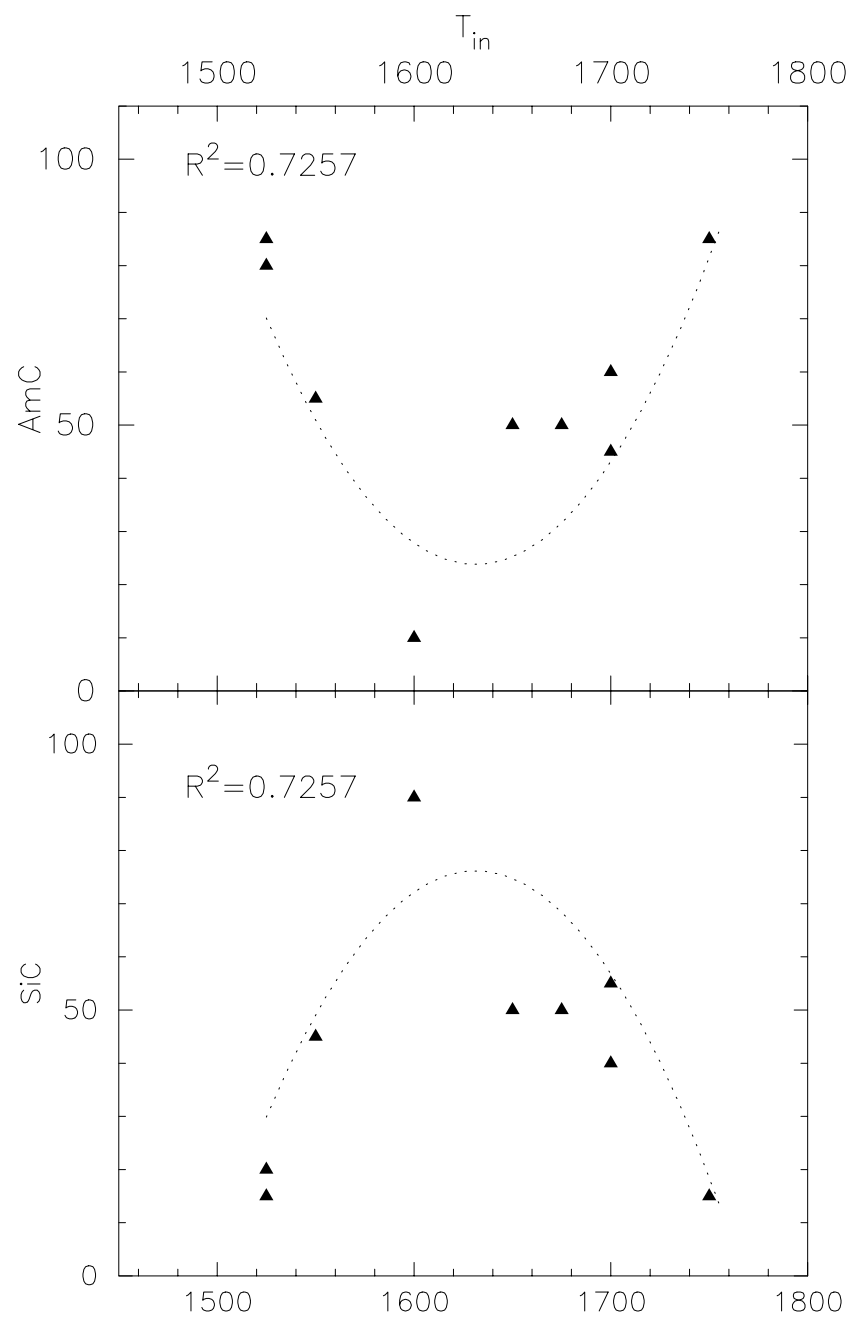

Figure 5.24: The correlations between the amount of AmC grains and the temperature of the inner dust shell and the amount of $\mathrm{SiC}$ grains and the temperature of the inner dust shell for AmC 2-shells models. 


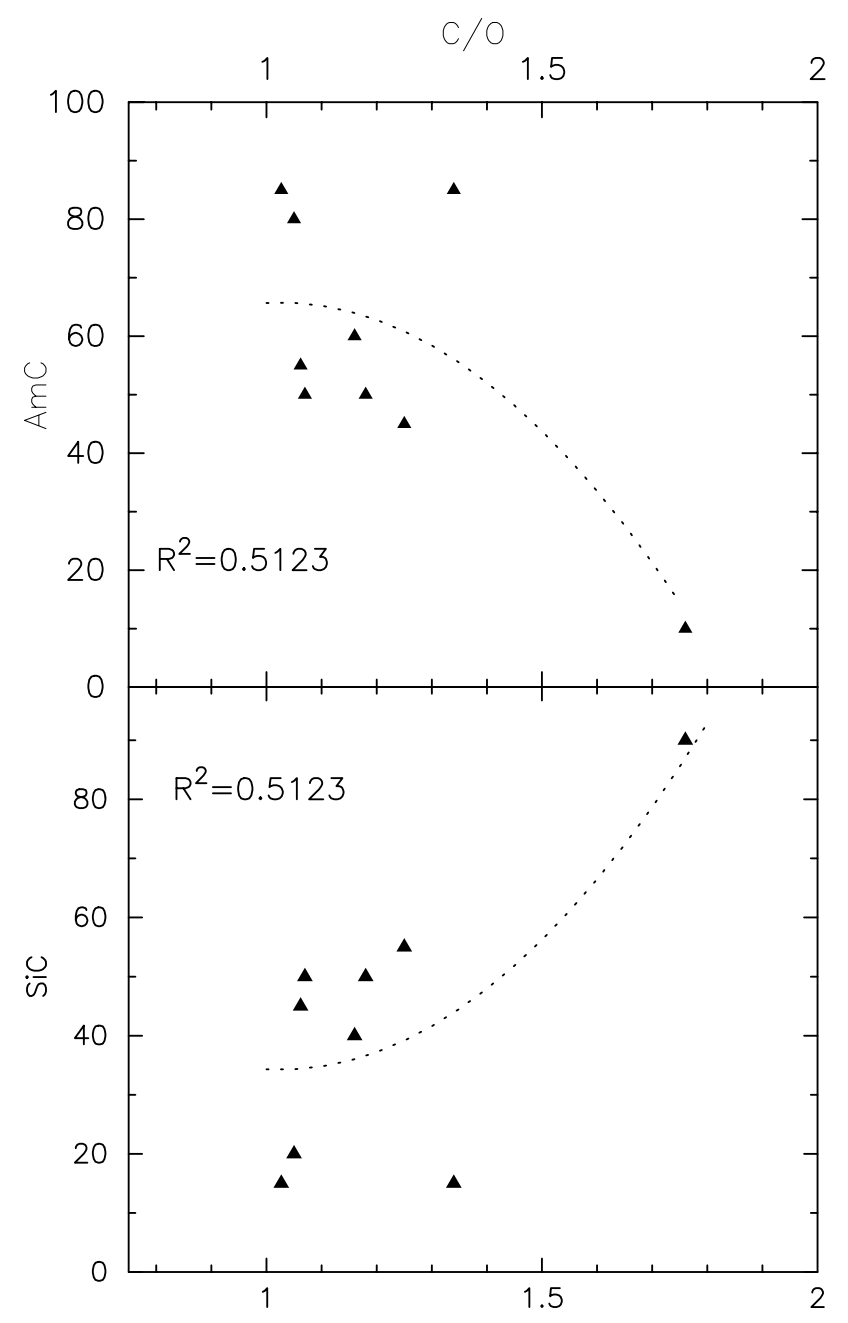

Figure 5.25: The correlations between the amount of $\mathrm{AmC}$ grains and the $\mathrm{C} / \mathrm{O}$ ratio and the amount of $\mathrm{SiC}$ grains and the $\mathrm{C} / \mathrm{O}$ ratio shell for $\mathrm{AmC} 2$-shells models. 


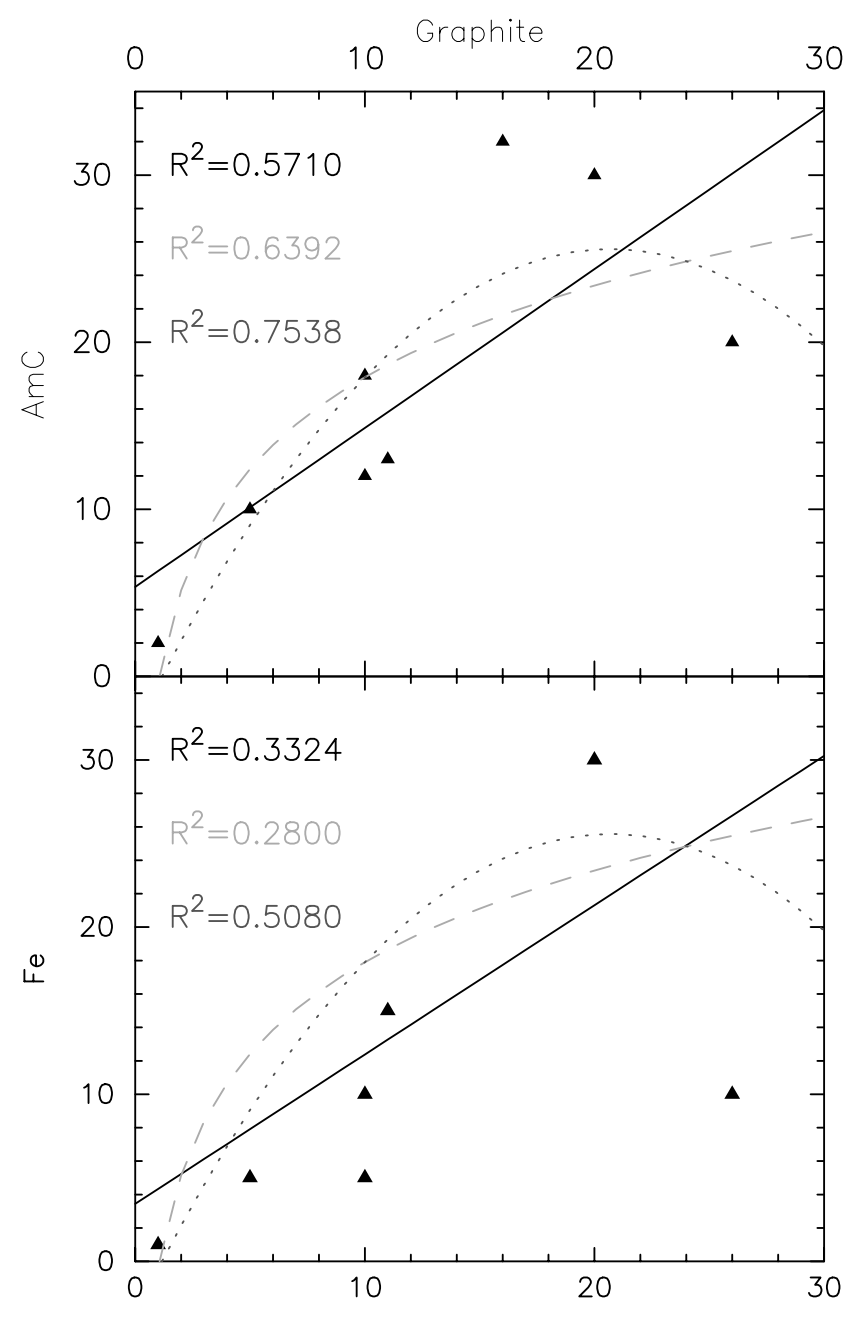

Figure 5.26: The correlations between the abundances $\mathrm{SiC}$ grains in $\mathrm{AmC}$ and graphite single shell models and abundances $\mathrm{SiC}$ grains in iron and graphite single shell models.as 


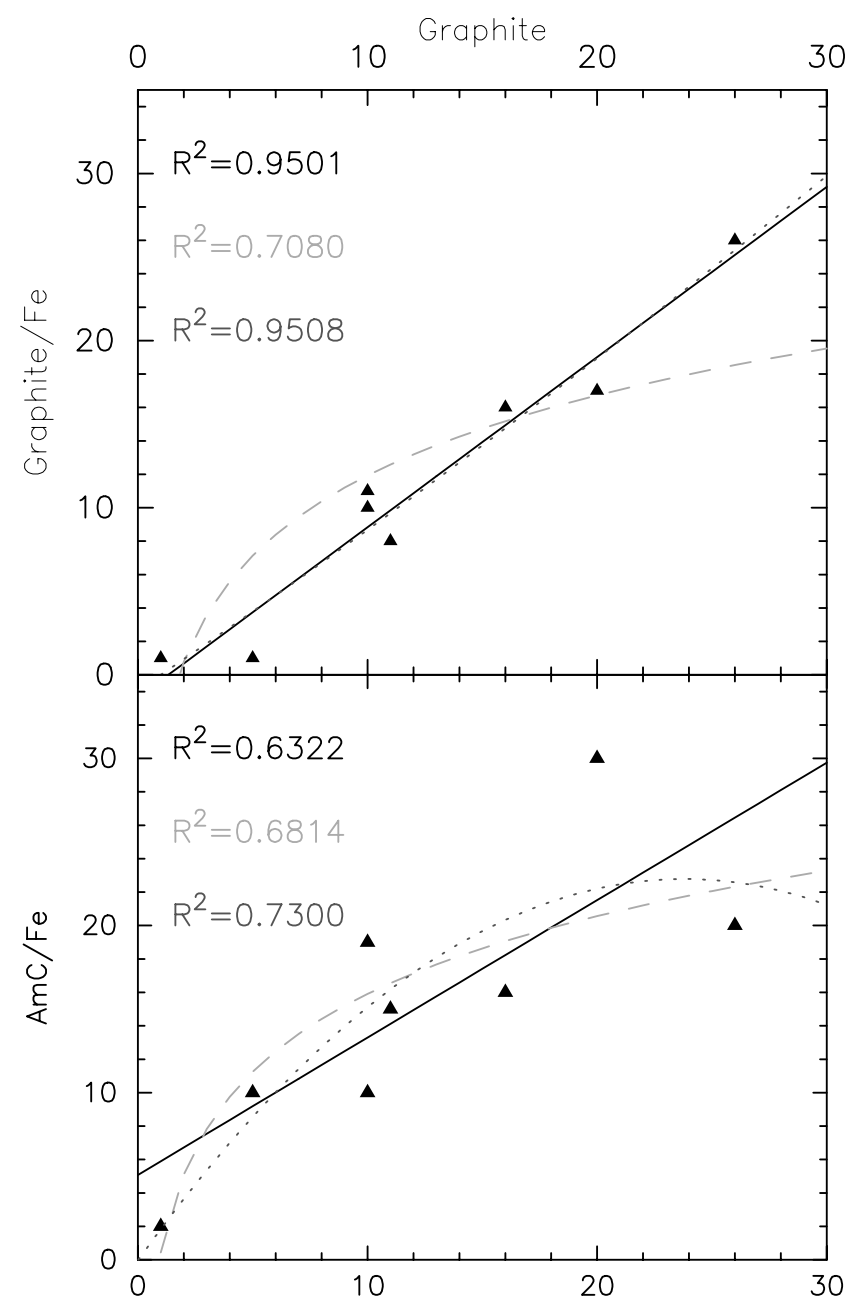

Figure 5.27: The correlations between the abundances $\mathrm{SiC}$ grains in graphite/Fe and graphite single shell models and abundances $\mathrm{SiC}$ grains in $\mathrm{AmC} / \mathrm{Fe}$ and graphite single shell models. 


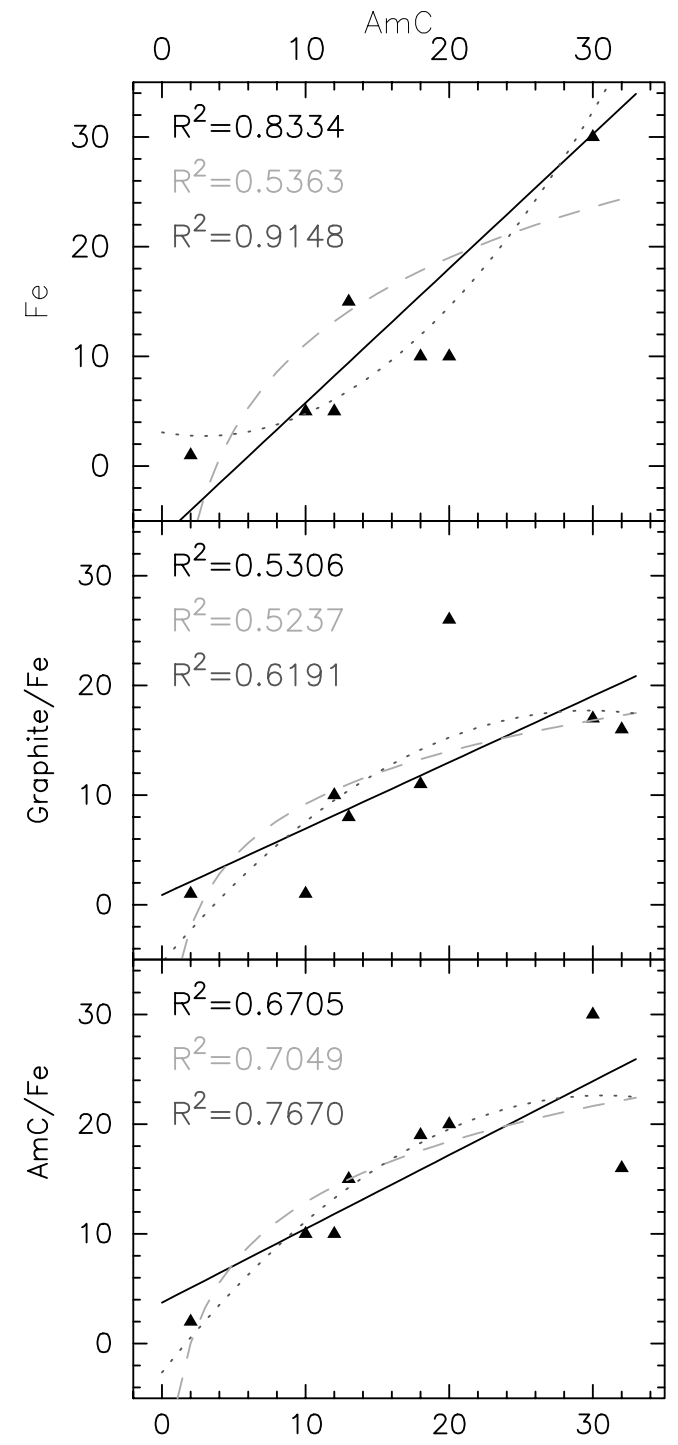

Figure 5.28: The correlations between the abundances $\mathrm{SiC}$ grains in $\mathrm{Fe}, \mathrm{AmC}$ single shell models and abundances $\mathrm{SiC}$ grains in graphite/Fe and $\mathrm{AmC}$ and abundances $\mathrm{SiC}$ grains in $\mathrm{AmC} / \mathrm{Fe}$ and $\mathrm{AmC}$ single shell models. 


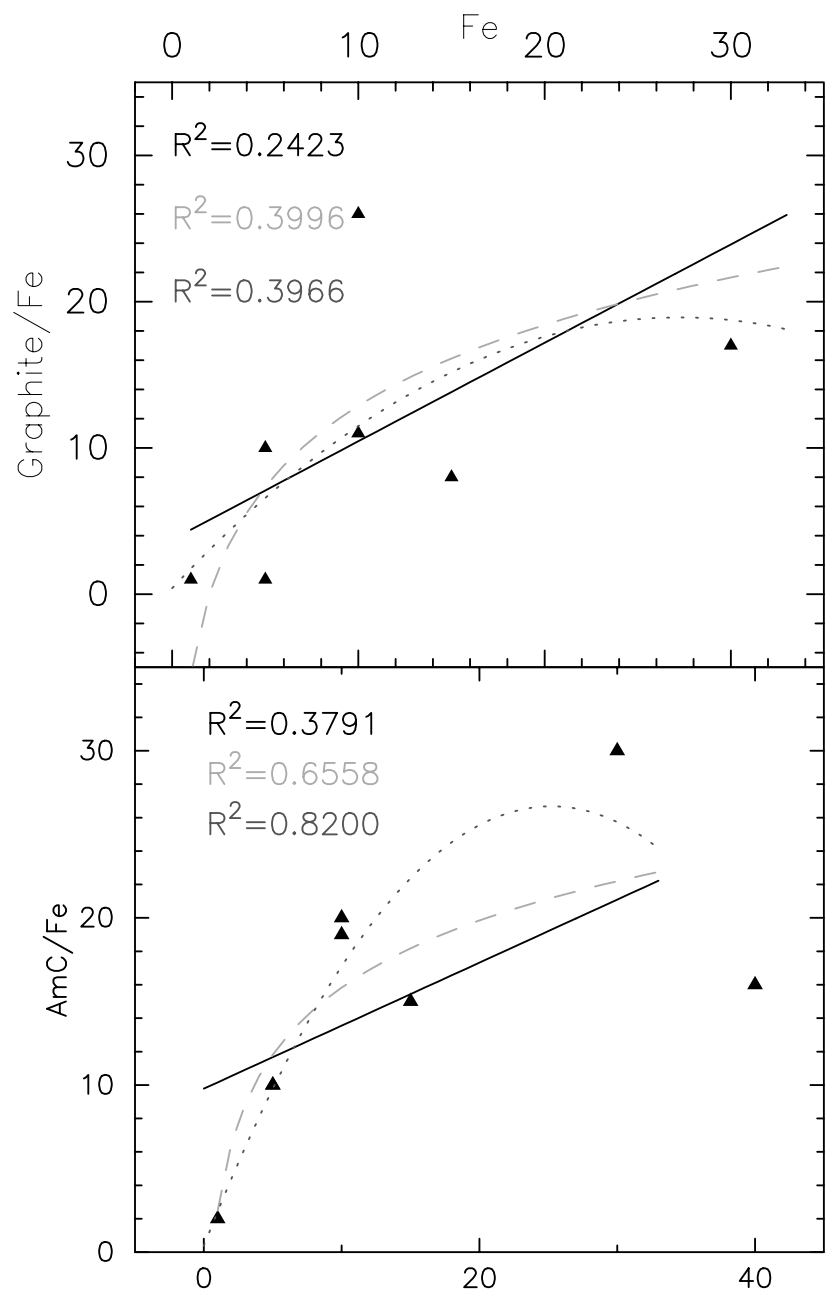

Figure 5.29: The correlations between the abundances $\mathrm{SiC}$ grains in graphite/Fe and iron single shell models and abundances $\mathrm{SiC}$ grains in $\mathrm{AmC} / \mathrm{Fe}$ and iron single shell models. 


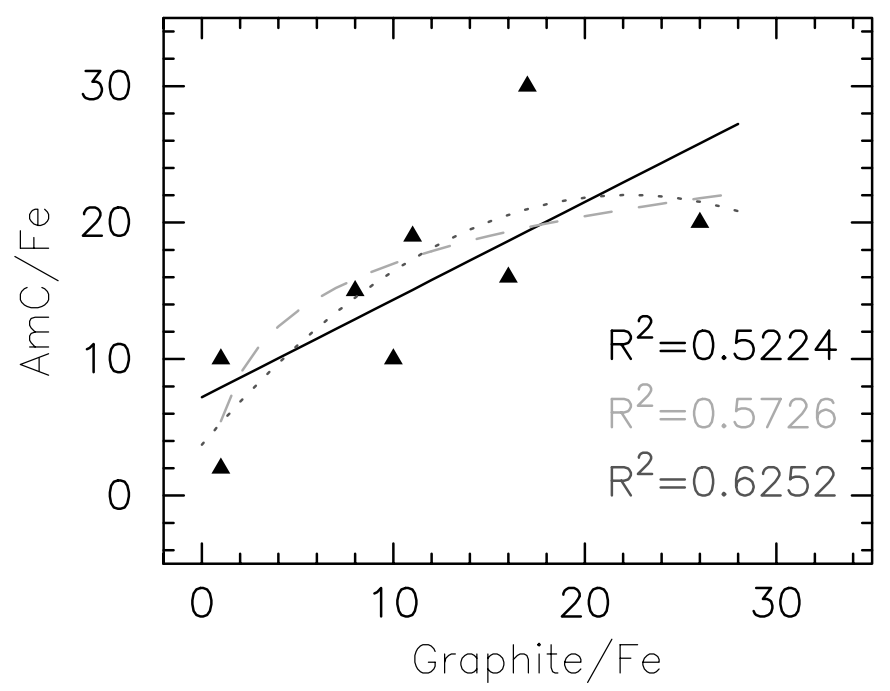

Figure 5.30: The correlations between the abundances $\mathrm{SiC}$ grains in $\mathrm{AmC} / \mathrm{Fe}$ and graphite/Fe single shell models for single shell models.

\subsubsection{Discussion}

In $\S 5.2$ we showed correlations between modeled and for collated parameters. In this section, we will discuss their significance. The inner dust temperature and the $\mathrm{C} / \mathrm{O}$ ratio are expected correlate since the $\mathrm{T}_{i n}$ is derived from the $\mathrm{P}-\mathrm{T}$ space Figure 4.1. Unity is not expected since determining the $T_{i n}$ depends on other parameters. These results do suggest that the P-T space is valuable for constraining RT model parameters.

There are number of correlations with a strong correlation. However, these correlations may or may not be true since they depend on the type of carbon used or the type of correlation. The graphite/Fe and $\mathrm{AmC} / \mathrm{Fe}$ models and their correlations with the amount of graphite, $\mathrm{AmC}$ and iron grains with the $\mathrm{Fe} / \mathrm{H}$ and the amount of graphite and $\mathrm{AmC}$ grains with the amount of iron grains, signification correlations 
exist for iron grains and graphite or AmC grains and graphite, AmC and iron grains with $\mathrm{Fe} / \mathrm{H}$, these are excepted since these parameters depend on each other since the amount of iron grains is derived from the $\mathrm{Fe} / \mathrm{H}$ and $\mathrm{C} / \mathrm{H}$ data (see Tables 4.15 and 5.16) . The gas-to-dust ratio $(\mathrm{g} / \mathrm{d})$ and optical depth $\left(\tau_{0.55 \mu m}\right)$ have a correlations (see Tables 4.15 and 5.16). The gas to dust tell how much gas to dust there is in the star's circumstellar shell and the optical depth $\left(\tau_{0.55 \mu m}\right)$ is the measure of density of dust, this would be expected since both variables involves the amount of dust in the star's shell. The gas-to-dust ratio is from previous studies (see Bergeat et al 2004) and $\tau_{0.55 \mu m}$ is related to the results of our models, these two model parameters are consistent with each other, suggesting our models agree with previous studies models.

We also found strong correlations in the abundance of $\mathrm{SiC}$ grains between the different models excluding those models in which iron is dominated, see Table 5.20. Since we saw correlations between the different types of carbon or iron used, these correlations suggest that there is no sensitivity to the precise nature of the continuum as long as the continuum is not dominated by iron grains. This agrees with previous studies that showed the choice of carbon grains is equivocal. However, the strength of SiC feature had no correlations between either modeled, derived or collated parameters. This is unexpected with previous studies using $\mathrm{SiC}$ grains and modeling the $\sim 11 \mu \mathrm{m}$ feature. High $\mathrm{C} / \mathrm{O}$ ratios in low-metallicity stars might prevent the formation of $\mathrm{SiC}$ grains and led to the condensation of graphite grains instead and increase of carbon and silicon the star envelopes for higher stellar mass and lower metallicity (Zinner 2006). 


\section{Chapter 6}

\section{Summary and Future Work}

To infinity and beyond- Buzz Lightyear (Toy Story, Toy Story 2 and Toy Story 3)

\subsection{Conclusion}

In $\S 4$, we examined a collection of nine carbon stars, examining their modeling parameters and other parameters of the star and dust shell. We found the following significant correlations for the single-shell modeling with graphite and iron grains and AmC and iron grains: (1) $\tau_{0.55 \mu m}$ and gas-to-dust ratio, (2) iron grains and graphite or AmC grains, (3) graphite or AmC grains and $\mathrm{Fe} / \mathrm{H}$, and (4) iron grains and $\mathrm{Fe} / \mathrm{H}$. For the collated data the significant correlations we found were: (1) for dust formation temperature and the change of temperature from the formation of graphite grains to the formation of $\mathrm{SiC}$ grains (2) $\mathrm{C} / \mathrm{O}$ and the change of temperature from 
the formation of graphite grains to the formation of $\mathrm{SiC}$ grains. Also, There was significant correlations in the abundance of $\mathrm{SiC}$ grains between the different models expect for when the abundance of $\mathrm{SiC}$ grains in iron models were compared to the other models $\mathrm{SiC}$ grains abundances. From this, we concluded that the continuum is not sensitive when choosing different types of carbon to model with as long as the continuum is not modeled with just iron and $\mathrm{SiC}$ grains.

\subsubsection{Future Work}

If more accurate radiative transfer models were fit to these star's spectra, it maybe be possible to obtain or find more precise or additional dust shell parameters, which will either help strengthen our results or show a way past the degenerate parameter space. Correlations may be stronger with less degeneracy. Also, if a larger set of stars were examined to have stronger statistics significance.

In sections $\oint 4.4 .2$ and 5.2, we examined a the inclusions of iron grains. We saw correlations with model parameters only with models with graphite and $\mathrm{AmC}$ with iron in the dust shell.

\subsubsection{Future Work}

Creating 2-shell models with graphite/Fe and AmC/Fe could lead to more accurate radiative transfer models and more correlations between the modeling parameters and other parameters. 


\subsubsection{Future Work}

Increasing the sample size of stars will decrease the margin or error, and increase the confidence level of the statistics. A larger sample size is more representative of the population, limiting the influence of outliers and/or extreme observations. 


\section{Bibliography}

Abia, C., Recio-Blanco, A., de Laverny, P., Cristallo, S., Dominguez, I., \&

Straniero, O. 2015, ApJ, 694, 2

Baron, Y., de Muizon, M., Papoular, R., \& Pegourie, B. 1987, A\&A, 186, 271

Bergeat, J. ,\& Chevallier, L. 2004, ApJ, 429, 235-246

Bernatwicz, T.J., Croat, T.K., \&, Daulton, T.I. 2006, Origin and Evolution of Carbonaceous Presolar Grains in Stellar Environments, ed. Lauretta, D.S. \&

McSween, H.Y., Jr., 109-126

Bohren, C., \& Huffman, D. 1983, Absorption and Scattering of Light by Small

Particles, 1st edn. (New York; Wiley)

Carroll, B.W., \& Ostlie, D.A. 1996, An Introduction to Modern Astrophysics

(New York; Addison-Wesley)

Chan, S. J., Kwok, S. 1990, A\&A, 237, 354

Cherchneff, I. 2006, A\&A, 456, 1001

Chigai, T., \& Yamamoto, T. 2003, Geochimica et Comsochimica Acta, 67, 64

Clayton, D.D., \& Nittler, L.R., 2004, A\&A, 42, 39

Cohen, M. 1984, MNRAS, 206, 137 
Comelli, G., Stohr, J., Robinson, C.J., \& Jark, W. 1988, Physical Review B, 38, 7511

Danilovich, T., Teyssier, D., Justanont, K., et al. 2015, A\&A, 581

Dominik, C., Gail, H.P., \& Sedimayr, E., 1989, A\&A, 233, 227

Draine, B.T., \& Lee, H.M. 1984, ApJ, 285, 89

Freedman, R., \& Kaufmann, W. 2005, Universe, 7th edn. (New York; W.H. Freeman and Company)

Fontaine, G., Brassard, P., \& Bergeron, P. 2001, Publication of the Astronomical Society of the Pacific, 113, 409

Goebel, J. H., Cheeseman, P., \& Gerbault, F. 1995, ApJ, 449, 246

Groenewegen, M.A.T. 1995, A\&, 293, 463

Groenewegen, M.A.T., Sevenster, M., Spoon, H.W.W., \& Perez, I. 2002, A\&A, 390,511

Hackwell, J.A. 1972, Astron. Astrophys. J. 200, 688

Hanner, M. 1988, Grain optical properties, Tech. rep.

Helling Ch., Woitke P. 2006, A\&A 455, 325

Huang, K 1987, Statistical Mechanics, 2nd edn. (Wiley)

Iben, I., \& Renzini, A., 1983, ARA\&A 21, 271

Ivezic, Z., \& Elitzur, M. 1995, ApJ, 445, 415

Ivezic, Z., \& Elitzur, M., 1997, MNRAS

Kelires, P.C. 1993, Physical Review B, 47, 1829

Kessler, M.F.. et al. 1996, A\&A, 315, 317

Kim, S., Martin, P.G., \& Hendry, P.D. 1994, ApJ, 422, 164 
Kipper, T. 1998, Baltic Astronomy, 7, 435-450

Kwok, S. 2004, Nature, 296, 132

Lagadec, E., et al. 2007, Monthly Notices of the Royal Astronomical Society, 376,1270

Lamers, H., \& Cassinelli, J. 1999, Introduction to Stellar Winds (Cambridge;

Cambridge University Press)

Leisenring, J.M., Kemper, F., \& Sloan, G.C. 2008, ApJ, 681, 1557

Lodders K., \& Fegley, B, 1995, Meteoritics 30, 661

Lodders K., \& Fegley, B, 1998, The Planetary Scientist's Companion, Oxford Univ. Press

Mathis, J.S., Rumpl W., \& Nordsiwck, K.H., 1977, ApJ, 217, 424-433

Matsuura, M., et al. 2005, A\&A, 217, 425

McDonald I., et al., 2011, ApJS, 193, 23

Messenger, S.J. et al. 2013, ApJ, 764, 142

Meyer, B. S. \& Zinner E. 2006, Nucleosynthesis, In Meteorites and the Early

Solar System II (eds. D. S. Lauretta and H. Y. McSween Jr.), University of Arizona, Tucson, 69-108

Nenkova, M., Ivezic, Z., \& Elitzur, M. 2000, Thermal Emission Spectroscopy and Analysis of Dust, Disks, and Regoliths, 196, 77 Neugebauer, G., et al. 1984, Science 224, 14

Pegourie, B. 1988, A\&A, 194, 335

Rowan-Robinson, M., and Harris, S. 1983, MNRAS 202, 767

Saada, D. 2000, PhD thesis, Israel Institute of Technology 
Sharp, C.M., \& Wasserburg, G.J. 1995, Geochimica et Cosmochimica Acta, 59, 1633

Sloan ,G.C., Devost, D., Bernard-Salas, J., Wood, P.R., \& Houck J.R. 2006, ApJ, 638, 472

Speck, A. K. 1998, The Mineralogy of Dust Around Evolved Stars, PhD thesis, University College London

Speck, A. K., Barlow, M. J., \& Skinner, C. J. 1997, MNRAS, 288, 431

Speck, A. K., Corman, A. B., Wakeman, K., Wheeler, C. H., \& Thompson, G. 2009, ApJ, 691, 1202

Speck, A. K., Pitman, K. M., \& Hofmeister, A. M. 2015, ApJ, 809, 65

Speck, A. K., Thompson, G. D., \& Hofmeister, A. M. 2005, ApJ, 634, 426

Stevenson, D. J., \& Salpeter, E. E. 1977, ApJS. 35, 239-261

Thompson G.D. et al. 2006, ApJ, 652, 1654

Treffers, R.R., \& Cohen, M. 1974 ApJ, 188, 545

Vassiliadis, E., Wood, P. R. 1993, A\&A, 413, 641

Villaver, E., Garcia-Segura, G., \& Manchado, A. 2002a, ApJ, 571, 880

Volk, K., Kwok, S.,\& Langill, P. P. 1992, ApJ, 391, 285

Willacy, K. 2003, ApJ, 600, 1

Willems, F. J. 1988, A\&A, 203, 51

Zinner, E., Nittler, L., Gallino, R., Karkasm A., Lugaro, M., Straniero, O., \& Lattanzio, J.C. 2006, ApJ, 650, 350 


\section{Vita}

\section{Hannah Groom}

Hannah Groom was born in Kansas City, Missouri. She didn’t own a T.V. while writing this. Also, she didn't invent the printing press, the internet or sliced bread. Hannah Groom, attended undergraduate school at Southeast Missouri State and then graduate school at the University of Missouri. Despite her decision to attend the University of Missouri, she stayed a faithful Kansas Jayhawk fan, Rock Chalk! This dissertation was written after six long years being an under-appreciated teaching assistant (aka glorified babysitter) and working for indentured servants wages.

P.S. No dinosaurs were around when this was written.

P.S.S. No animals were hurt in the making of this book. 